Aus dem Institut für Tierzucht und Haustiergenetik der Georg-August-Universität Göttingen

\title{
Die Implementierung der Selektion gegen Osteochondrose in ein Zuchtprogramm beim Warmblutpferd
}

\author{
Dissertation \\ zur Erlangung des Doktorgrads \\ der Fakultät für Agrarwissenschaften \\ der Georg-August-Universität Göttingen
}

vorgelegt von

Stephanie - Mabinti Busche

geboren in Freetown / Sierra Leone

Göttingen, im November 2005 
D 7

Referent:

Prof. Dr. E. Bruns

Korreferent:

Prof. Dr. Dr. M. Gauly

Tag der mündlichen Prüfung: $\quad$ 18.11.2005 
Meiner Familie 



\section{Inhaltsverzeichnis}

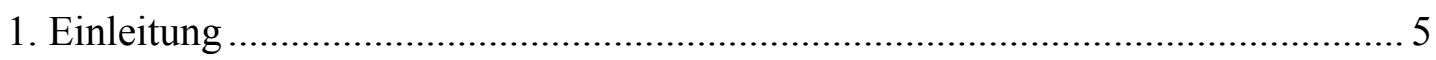

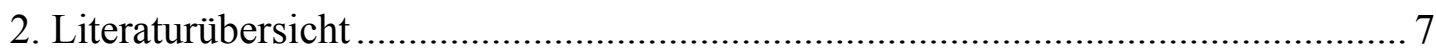

2.1. Bedeutung der Gliedmaßenerkrankungen in der Reitpferdezucht.................... 7

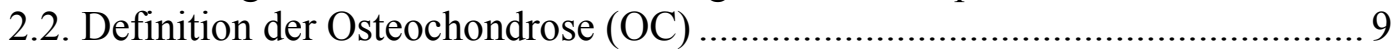

2.2.1. Osteochondrosis dissecans (OCD) .................................................... 9

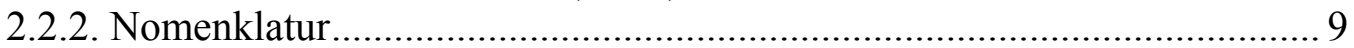

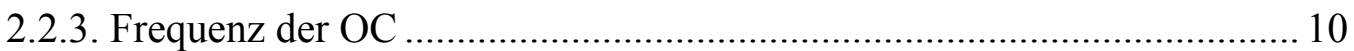

2.3. Die Knochenentwicklung.......................................................................... 12

2.3.1. Störungen der Knochenentwicklung/ Skelettreifung ................................ 13

2.3.2. Störungen der Knochenentwicklung im Rahmen der OC......................... 14

2.3.3. Störungen der Knochenentwicklung im Rahmen der OCD ...................... 15

2.4. Krankheitsverlauf/Klinische Symptome .................................................... 15

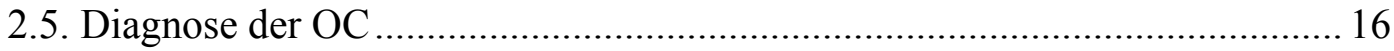

2.5.1. Diagnose der OC anhand von Röntgenbildern ........................................ 16

2.5.2. Diagnose der OC durch molekulargenetische Analysen.......................... 18

2.6. Einflussfaktoren auf die Entstehung der OC /OCD ........................................ 19

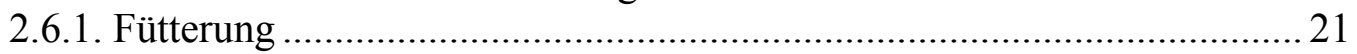

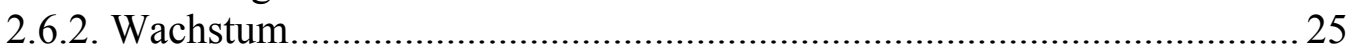

2.6.3. Bewegung / Mechanische Belastung ………………………………….....2 29

2.6.4. Endokrinologie des Knochenstoffwechsels ............................................... 31

2.6.5. Genetische Einflussfaktoren ...................................................................... 33

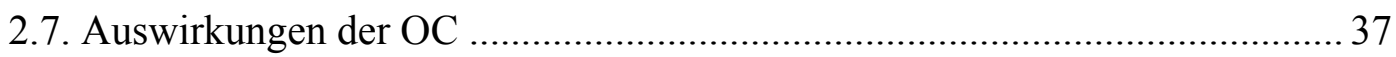

2.7.1. Beziehungen zwischen orthopädischen Defekten und Leistungs- sowie

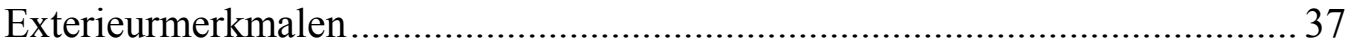

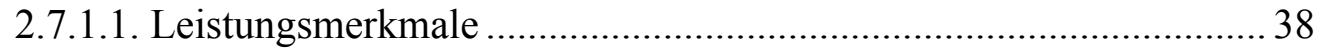

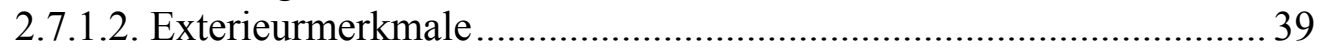

2.7.2. Einfluss der OC auf die Nutzungsdauer von Sportpferden........................ 40

2.7.3. Wirtschaftliche Bedeutung der OC ......................................................... 41

2.8. Zuchtplanung in der deutschen Reitpferdezucht........................................... 42

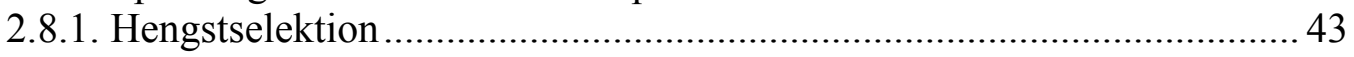

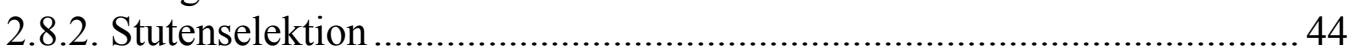

2.8.3. Zuchtwertschätzung in der Reitpferdezucht............................................ 46

2.8.4. Die Implementierung von Gesundheitsmerkmalen in Tierzuchtprogramme .48

2.8.5. Die Implementierung der Selektion gegen OC in Pferdezuchtprogramme

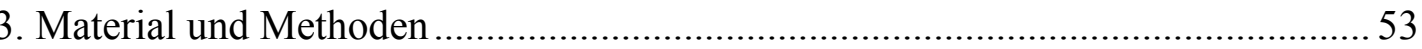

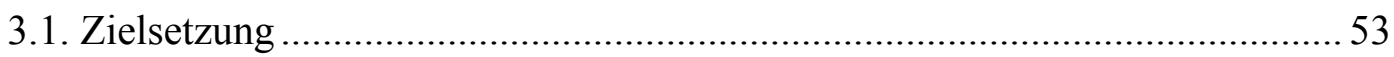

3.2. Einsatzmöglichkeiten der Computersimulation ...............................................53

3.3. Beschreibung des Simulationsprogramms ………………………………...... 55

3.3.1. Erstellung der simulierten Leistungsdaten ................................................. 55

3.3.2. Erstellung der simulierten Population ....................................................... 57

3.3.3. Parameter der simulierten Population ..................................................... 58

3.4. Entwicklung der genetischen Modelle............................................................. 59 


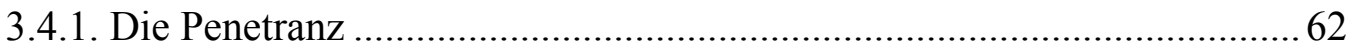

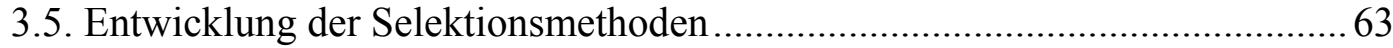

3.6. Simulation verschiedener Selektionsszenarien gegen OC aus der

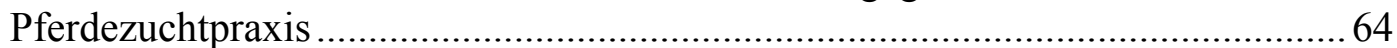

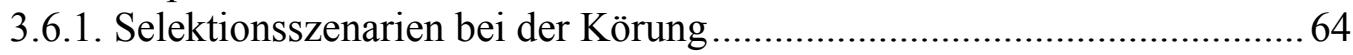

3.6.2. Weitere simulierte Selektionsszenarien ..................................................6 68

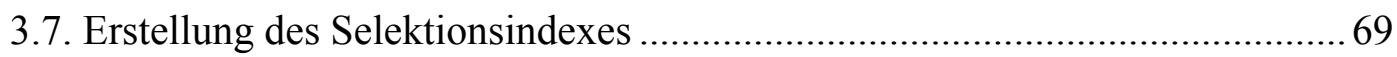

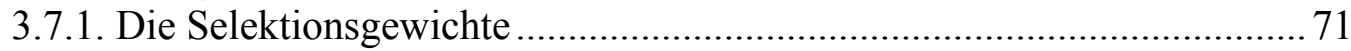

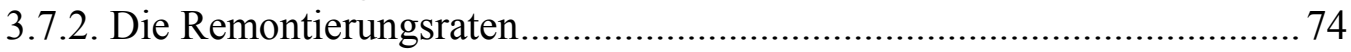

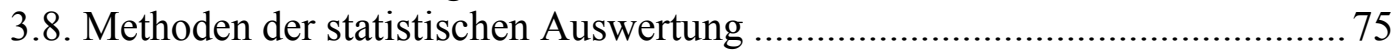

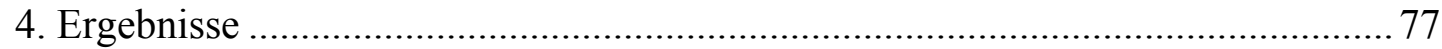

4.1. Reduktion der OC-Frequenz bei Anwendung der genetischen Modelle I und II innerhalb der einstufigen Selektion........................................................................ 77 4.2. Reduktion der OC-Frequenz bei Anwendung der genetischen Modelle I und II

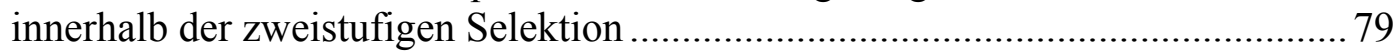
4.3. Reduktion der OC-Frequenz bei Anwendung der ein-oder zweistufigen

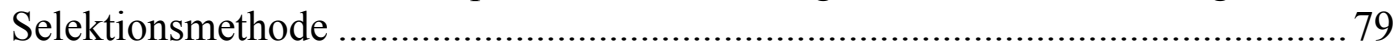
4.4.Veränderungen der Zuchtwerte der Leistungsmerkmale bei unterschiedlicher

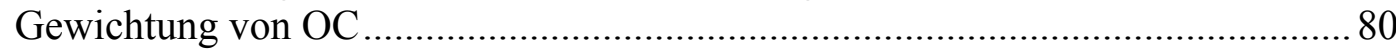
4.5. Einfluss der Remontierungsraten auf die Reduktion der OC-Frequenz und die

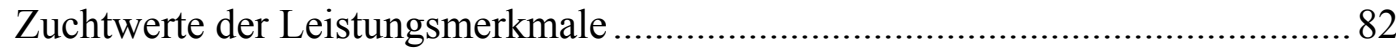
4.6. Simulation verschiedener Selektionsszenarien gegen OC aus der

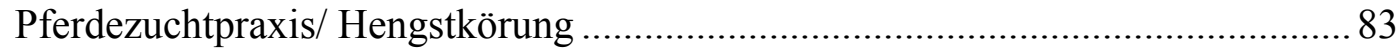

4.6.1.a. Modell mit vier Klassen ................................................................. 84

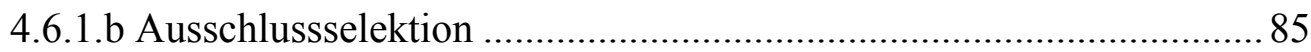

4.6.1.1. Vergleich des Modells mit vier Klassen und der Ausschlussselektion

4.6.1.c. Zuchtwertschätzung für OC

4.6.2. Vergleich der Selektionsszenarien /Hengstkörung .................................. 90

4.6.3. Simulation weiterer Selektionsszenarien gegen OC ….......................... 92

4.6.3.1. Vorhandensein eines Gentests für OC ........................................... 92

4.6.3.2. Zusätzliche Einbeziehung der Stuten in die Selektion gegen OC .....93

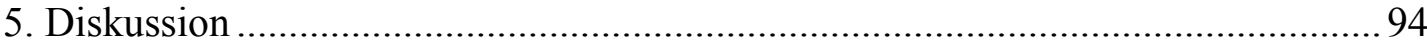

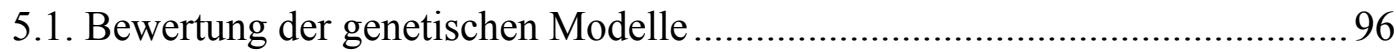

5.2. Bewertung der Selektionsmethoden.......................................................... 99

5.2.1. Einfluss der Selektionsgewichte auf die Selektion gegen OC und die

Zuchtwerte der Leistungsmerkmale ............................................................. 100

5.2.2. Einfluss der Remontierungsraten auf die OC-Frequenz und die

Zuchtwerte der Leistungsmerkmale ............................................................... 102

5.3. Bewertung verschiedener simulierter Selektionsszenarien gegen OC ........... 104

5.3.1. Bewertung verschiedener simulierter Selektionsszenarien bei der

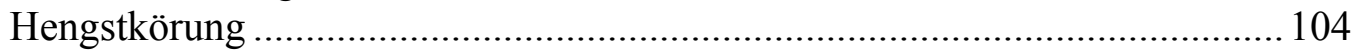

5.3.2. Bewertung weiterer simulierter Selektionsszenarien ........................... 121

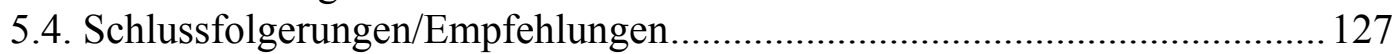

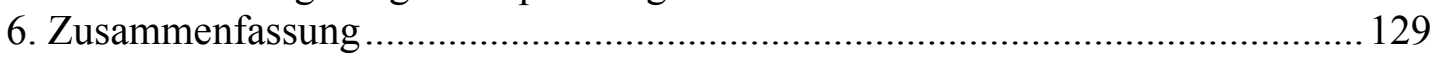

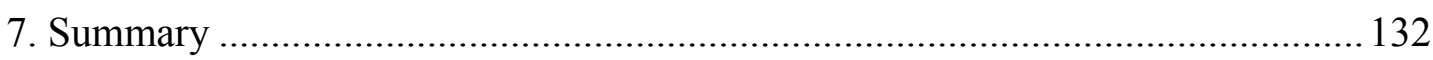

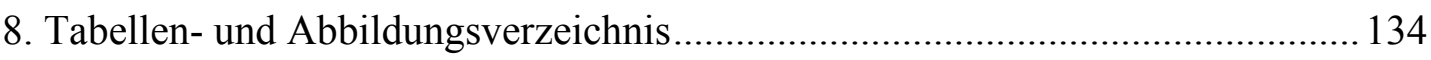

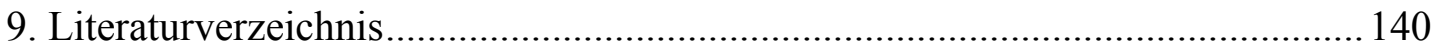




\section{Abkürzungsverzeichnis}

\begin{tabular}{|c|c|}
\hline BLUP & Best Linear Unbiased Prediction \\
\hline $\mathrm{BMC}$ & Bone Mineral Content \\
\hline BMD & Bone Mineral Density \\
\hline bzw. & beziehungsweise \\
\hline ca. & circa \\
\hline $\mathrm{CC}$ & Contemporary Comparison \\
\hline DOD & Developmental Orthopaedic Disease \\
\hline FN & Fédération National- Deutsche Reiterliche Vereinigung e.V. \\
\hline GFE & Gesellschaft für Ernährungsphysiologie \\
\hline ggf. & gegebenenfalls \\
\hline GOT & Gebührenordnung für Tierärzte \\
\hline $\mathrm{HG}$ & Halbgeschwister \\
\hline HLP & Hengstleistungsprüfung \\
\hline HYPP & Hyperkalaemic Periodic Paralysis \\
\hline ICTP & Carboxyterminales Telopeptid des TypI Kollagens \\
\hline IGF & Insulin Like Growth factors \\
\hline KWPN & Koninklijk Warmbloed Paardenstammboek Nederland \\
\hline NRC & National Research Council \\
\hline $\mathrm{OC}$ & Osteochondrose \\
\hline OCD & Osteochondrosis dissecans \\
\hline OLWS & Overo Lethal White Syndrom \\
\hline PICP & Carboxyterminales Propeptid des Typ-I Kollagens \\
\hline PTH & Parathormon \\
\hline QTL & Quantitative Trait Loci \\
\hline
\end{tabular}


SCID Severe Combined Immunodefiency

tz

tägliche Zunahme

u.a. unter anderem

vs. versus, kontra

z.B. zum Beispiel

ZVO Zuchtverbandsordnung 


\section{Einleitung}

In der heutigen Reitpferdezucht gewinnen Gesundheitsmerkmale eine immer größere Bedeutung. Auf der Käuferseite werden nicht nur in sportlicher Hinsicht qualitativ hochveranlagte Pferde verlangt, sie müssen auch den Anforderungen, die im Freizeitund Turniersport gestellt werden, gesundheitlich standhalten können (WINTER, 1995). Reitpferde sind durch intensives Training, vermehrte Turnierteilnahme und steigende Transportbelastungen vielschichtigen Belastungen ausgesetzt (GLODEK, 1979). Hinzu kommt, dass Sportpferde erst mit ca. 10 Jahren ihren Leistungshöhepunkt erreicht haben (PHILIPSSON, 1999). Bis zu diesem Zeitpunkt sind bereits erhebliche Investitionen für die Ausbildung erbracht worden. Deshalb ist es auch aus finanzieller Hinsicht wichtig, Pferde zu züchten, die über eine gesunde Konstitution verfügen, um ihr Leistungspotenzial voll ausschöpfen zu können. Eine lange Nutzungsdauer ist demnach in allen Bereichen des Pferdesports unerlässlich, und nicht zuletzt verlangt der Tierschutz, dass Aspekte der Gesundheit in der Tierzucht Berücksichtigung finden. Diesen Anforderungen muss durch züchterische Überlegungen und vermehrte Integration von Gesundheitsmerkmalen in Zuchtprogramme entsprochen werden. Die Integration von funktionalen Merkmalen in Zuchtprogramme wird zwar als wichtig erachtet, bisher wurden sie aber nur ansatzweise in züchterische Entscheidungsprozesse eingebunden. Die problematische wirtschaftliche Situation und die erschwerte Vermarktungssituation auf dem Pferdemarkt machen eine verstärkte Berücksichtigung von Gesundheitsmerkmalen bei züchterischen Entscheidungen aber dringend notwendig.

$\mathrm{Zu}$ den bedeutenden Erkrankungen in der Sportpferdezucht, die häufig zum Ausscheiden der Pferde aus dem Sport oder zu dauernder Unbrauchbarkeit führen können, gehören Erkrankungen der Gliedmaßen. Eine besondere Stellung nimmt hier die Osteochondrose ein, die in den vergangenen Jahren eine häufige Diagnose bei Ankaufsuntersuchungen darstellte. 
Im Rahmen dieser Arbeit wurde ein Computersimulationsprogramm entwickelt, anhand dessen untersucht werden soll, mit welchen züchterischen Maßnahmen die Frequenz der Ostechondrose gesenkt werden kann. Von besonderem züchterischem Interesse ist in diesem Zusammenhang die Frage, ob und wie sich die Zuchtwerte der Leistungsmerkmale bei einer Einbeziehung der Osteochondrose in Selektionsentscheidungen verändern. Die Beantwortung dieser Frage ist ebenfalls Teil dieser Untersuchung.

Mit Hilfe des entwickelten Programms soll es letztendlich möglich sein, die Auswirkungen von Selektionsstrategien gegen Osteochondrose auf eine Pferdepopulation in kurzer Zeit nachvollziehen zu können und damit Empfehlungen an Zuchtverbände geben zu können, wie und in welcher Form die Selektion gegen Osteochondrose in ein Zuchtprogramm integriert werde sollte, um möglichst rasch große Zuchterfolge auf diesem Gebiet erzielen zu können. 


\section{Literaturübersicht}

\subsection{Bedeutung der Gliedmaßenerkrankungen in der Reitpferdezucht}

$\mathrm{Zu}$ den bedeutenden, in der Warmblutzucht auftretenden Erkrankungen zählen die Gliedmaßenerkrankungen, die häufig zum Ausscheiden der Pferde aus dem Sport oder sogar zu deren Unbrauchbarkeit führen können.

Die Relevanz von Gliedmaßendefekten als Abgangsursache bei Sportpferden, die bereits Thema zahlreicher Untersuchungen war, wird durch die unten aufgeführte Übersicht verdeutlicht.

Tabelle 1: Abgangsursachen und deren Häufigkeiten bei Sportpferden

\begin{tabular}{|lcccc|}
\hline \multicolumn{5}{c|}{ Häufigkeit erkrankter Pferde (\%) } \\
\hline $\begin{array}{l}\text { Erkrankung von } \\
\text { Organsystemen }\end{array}$ & $\begin{array}{c}\text { Clausen et } \\
\text { al. } \\
(1990)\end{array}$ & $\begin{array}{c}\text { Lindner\& } \\
\text { Offeney } \\
(1992)\end{array}$ & $\begin{array}{c}\text { Hommerich } \\
(1995)\end{array}$ & $\begin{array}{c}\text { Wallin et al. } \\
(2000)\end{array}$ \\
\hline Bewegungsapparat & 61,2 & 40,5 & 51,4 & 60,1 \\
Verdauungsapparat & 9,8 & 9,0 & 14,4 & 5,6 \\
Herz- & 4,6 & & 8,1 & 2,6 \\
/Kreislaufapparat & 1,2 & & 5,2 & 4,1 \\
Harnsystem & 16,3 & 16,5 & 12,2 & 8,9 \\
Atmungsapparat & 2,8 & & 2,3 & 5,4 \\
Infekt./ & & & & \\
Nervensystem & & & & \\
\hline
\end{tabular}

Mängel im Bewegungsapparat erwiesen sich durchweg als Hauptabgangsursache, während andere Erkrankungen des Organsystems deutliche geringere Prozentsätze aufweisen. HOMMERICH (1995) differenzierte die Erkrankungen des Bewegungsapparates genauer und gibt den Anteil akuter und chronischer Gelenksentzündungen mit $69 \%$ als häufigste Ursache in diesem Bereich an, gefolgt von Sehnen- und Sehnenscheidenentzündungen (5,5\%), Luxationen und Knochenbrüche (7,6\%) sowie Hufrehe und Rehhuf (2,4\%). 
Auch in der Studie von CLAUSEN (1990) machten akute und chronische Gelenkserkrankungen mit $38,1 \%$ den größten Anteil bei unterschiedlichen Gliedmaßenerkrankungen aus. Ergebnisse von GUTEKUNST (1977) zeigten, dass orthopädische Defekte bei 50-60\% der versicherten Pferde als Abgangsursache anzusehen waren. Auch KÖNING (1983) und BUTLER (1984) kamen zu ähnlichen Resultaten. Im Gegensatz dazu offenbarte eine Studie von BAKER und ELLIS (1981), dass im Vergleich zu anderen Erkrankungen offenbar deutlich weniger Pferde aufgrund von Gliedmaßenerkrankungen getötet werden. Im Rahmen dieser Untersuchung wurden 480 Pferde postmortal untersucht und festgestellt, dass nur 15,8\% der Tiere aufgrund von Defekten der Bewegungsorgane, hauptsächlich Frakturen getötet wurden. VELTJENS (1985) untersuchte anhand von 558 Pferde, die von 32 Warmbluthengsten aus verschiedenen Zuchtgebieten abstammten, die Häufigkeiten bestimmter Erkrankungen. 32,4\% der Abgänge hatten orthopädische Defekte als Ursache. PETZOLD et al. (1988) beschäftigten sich mit den Abgangsursachen von Englischen Vollblütern aus dem Renngeschehen und zeigten, dass $91 \%$ aller Tiere aufgrund von Gliedmaßenerkrankungen ausschieden. WALLIN (2000) kam in seiner Studie, die am Schwedischen Warm- und Kaltblut durchgeführt wurde, zu ähnlichen Ergebnissen. Erkrankungen des Muskel- und Skelettsystems wurden als Hauptgrund für Abgänge mit einem Prozentsatz von 57,2 identifiziert.

Die Ergebnisse zeigen, dass vor allem Gliedmaßendefekte häufig zum Ausscheiden von Sport- oder Rennpferden aus dem Sport führen. Dies führt zu finanziellen Verlusten im Hinblick auf Gewinngelder, aber auch der Handel von Pferden, die röntgenologische Auffälligkeiten zeigen, ist von Absatzschwierigkeiten und Mindereinnahmen beeinflusst. Eine Erkrankung, deren Auftreten in den letzten Jahren vermehrt zu den genannten Problemen für den praktischen Pferdezüchter und Verkäufer geführt hat, ist die Osteochondrose. Aus ökonomischen, aber auch aus tierschützerischern Gründen sollte eine Anpassung an die oben genannte Entwicklung stattfinden und Gesundheitsaspekte, vor allem solche, die die Gliedmaßen betreffen, stärker in den Mittelpunkt des züchterischen Interesses gerückt werden. 
Aufgrund dieser Entwicklungen hat die vorliegende Arbeit das Ziel, Selektionsstrategien gegen die Osteochondrose im Rahmen einer Computersimulation $\mathrm{zu}$ erproben und so mögliche züchterische Konsequenzen herauszuarbeiten, die zu einer Reduktion der Osteochondrose Frequenz in der Population führen.

\subsection{Definition der Osteochondrose (OC)}

Die Erkrankung Osteochondrose beim Pferd findet in der Literatur erstmals 1947 Erwähnung (NILSON, 1947). Osteochondrose ist ein allgemeiner Ausdruck für eine Krankheit bei Jungtieren, die aus einem gestörten Wachstum des Knorpels resultiert. Hauptsächlich sind die gewichttragenden Gelenke wie das Sprung- Fessel- und Kniegelenk betroffen (JEFFCOTT, 1991).

\subsubsection{Osteochondrosis dissecans (OCD)}

Bei der Osteochondrosis dissecans handelt es sich um eine spezielle Form der Osteochondrose. Durch die Störung der Verknöcherung entsteht ein übertrieben dickes Knorpelgewebe, dass von der Gelenkflüssigkeit nicht mehr ernährt werden kann. Dieses krankhaft verdickte Knorpelgewebe ist anfälliger für mechanische Verletzungen, es kann sich somit vom Knochen abspalten und Fragmente bilden, die im Gelenk frei beweglich sein können (NIXON, 1993).

\subsubsection{Nomenklatur}

In der Literatur werden unterschiedliche Begriffe und Bezeichnungen für den Begriff der Osteochondrosis dissecans verwendet (HERTSCH und HÖPPNER, 1992):

- Isolierte Verschattung (KROLL, 1999)

- Chip (Mc ILWRAITH, 1986)

- Gelenkmaus (EDWARDS, 1984)

- Sequester (HERTSCH, 1991)

- Gelenkkörper (STÖCKLI und UELTSCHI, 1992)

- Gelenkstein (HERTSCH und HÖPPNER, 1999) 
Auch der Begriff „Osteochondrose“ wird in der Literatur unterschiedlich bewertet: JEFFCOTT (1993) hält den Begriff „Dyschondroplasie“ für geeigneter, um die im wachsenden Knorpel entstehenden Läsionen zu beschreiben. DÄMMRICH (1985) ist der Meinung, dass die Veränderungen pathogenetisch richtiger als „Chondrosis dissecans“ bezeichnet werden sollten, da die Gelenkveränderungen im Gelenkknorpel beginnen. HERTSCH (1992) sieht die Ursache dieser unterschiedlichen Bezeichnungen in einer Vermischung von röntgenologischem Befund, patho-morphologischer oder klinischer Diagnose und möglicher Ätiologie.

\subsubsection{Frequenz der OC}

Allgemein wird in der Literatur aufgrund verschiedener Untersuchungen eine Frequenz der Osteochondrose in verschiedenen Populationen und an verschiedenen Lokalisationen von 10-30\% angenommen (WAGNER von MATTHIESEN, 1994; BRUNS, 2001). Diese Annahme konnte anhand der Ergebnisse verschiedener Studien bestätigt werden. SAMY (1977) diagnostizierte anhand der Röntgenbilder von 127 Pferden, bei denen der Verdacht einer Sprunggelenkserkrankung bestand, bei $11 \%$ eine Osteochondrosis dissecans. Röntgenologische Kontrolluntersuchungen von 150 ,gesunden“ Warmblütern ergaben sogar einen Anteil von 12\%. SAMY (1977) stellte weiterhin fest, dass die Erkrankung häufiger bei Warmblütern, Trabern und Vollblütern vorkommt und der überwiegende Teil der betroffenen Pferde im Alter bis 4 Jahren war. STAECKER (1987) untersuchte an Röntgenaufnahmen von 2.576 Pferden das Auftreten der Osteochondrose am Fesselgelenk und kam auf einen Anteil von insgesamt $13 \%$ bei Warmblütern, obwohl nur 38\% der Gelenke untersucht wurden. Untersuchungen von GRONDAHL (1992) an 753 Norwegischen Trabern zeigten eine Befundhäufigkeit von 14,3\% im Sprunggelenk und 11,8\% im Fesselgelenk. HEINZ (1993) führte eine Reihenuntersuchung an den Zehen und Sprunggelenken von 220 unselektierten Holsteiner Absatzfohlen durch. Er kam zu dem Ergebnis, dass 11,4 \% der Fohlen Befunde im Fesselgelenk zeigten und 8,7\% Veränderung im Sprunggelenk aufwiesen. Der niederländische Warmblutzuchtverband (KWPN), führte 1994 eine Studie an einer Stichprobe von 30 Hengsten durch, bei der sich eine OC-Frequenz von 18\% im Sprunggelenk ergab. Zusätzlich wurden 590 weibliche Nachkommen dieser Hengste untersucht und man fand bei $13,7 \%$ positive Befunde im Sprunggelenk. 
Ebenfalls an 135 holländischen Warmblütern fanden BARNEVELD und VAN WEEREN (1998) eine Gesamtfrequenz osteochondrotischer Veränderungen an der Lokalisation Sprung- und/oder Kniegelenk im Bereich von 20-26\%. Untersuchungen von BREHM und STAECKER (2000) an 1190 Trabrennpferden ergaben eine Befundhäufigkeit von 12,4\% im Sprunggelenk. RICARD et al. (2002) konnten in ihrer Untersuchung an 733 Pferden der Rasse Selle Français ein deutliches Auftreten osteochondrotischer Veränderungen an den verschiedenen Lokalisationen nachweisen. Frequenzen von 19\% im Fessel-, 24\% im Sprung- und 8\% im Kniegelenk wurden hier nachgewiesen. Im Rahmen einer groß angelegten Studie wurden in den Jahren 2001-2003 630 unselektierte Fohlen im Alter zwischen vier und neun Monaten und deren Mütter röntgenologisch untersucht. Von diesen 630 Fohlen wurden 405 im Alter von zwei Jahren einer nochmaligen röntgenologischen Überprüfung unterzogen. ARNAN (2003) kam anhand dieses Datenmaterials zu folgenden Ergebnissen: die OCD Häufigkeit in allen drei Gelenktypen ist hoch: 132 Fohlen $(32,6 \%)$ und 106 Zweijährige $(26,2 \%)$ waren positiv. Das Fesselgelenk war das am häufigsten betroffene Gelenk, 19,5\% der Fohlen und 16,6\% der Zweijährigen zeigten hier positive Befunde, gefolgt vom Sprunggelenk, hier fanden sich eine Osteochondrose Frequenz von $11,1 \%$ bei den Fohlen und 10,4\% bei den Zweijährigen. Das Knie erwies sich als das am wenigsten betroffenen Gelenk mit 7,2\% der Fohlen und 2,2\% der Zweijährigen. Ergebnisse von WITTWER et al. (2004) an 118 süddeutschen Kaltblutfohlen zeigten eine Frequenz von röntgenologischen Veränderungen, die sich dem Osteochondrose Komplex zuordnen lassen, von 59\%. Von diesen 118 Fohlen zeigten 61,8\% (73 Fohlen) röntgenologische Verschattungen. ROBERT et al. (2005) untersuchten Rassenunterschiede beim Auftreten von osteochondralen Veränderungen bei Absetzern in Frankreich. 133 Vollblüter, 161 französische Traber und 99 Selle Français Fohlen wurden zum Zeitpunkt des Absetzens röntgenologisch untersucht. Die Röntgenbilder wurden von drei Veterinärmedizinern analysiert und die Befunde klassifiziert. Die Fohlen der Rasse Selle Français wiesen generell schwerere Befunde auf als die Traber- und Vollblutfohlen $(\mathrm{p}<0,001)$. Bei der Betrachtung der lokalen Verteilung der Befunde zeigte die Rasse Selle Français mehr Befunde in den vorderen Fesselgelenken, im dorsalen Teil der hinteren Fesselgelenke und im Kniegelenk. Die Traberfohlen wiesen gehäuft Befunde im plantaren Teil der hinteren Fesselgelenke sowie im Vorderfußwurzelgelenk auf. 


\subsection{Die Knochenentwicklung}

Das Skelett des Pferdes wird bereits in einer sehr frühen Entwicklungsphase im Mutterleib angelegt (HERTSCH, 1999). Es besteht zunächst aus knorpeligen Vorstufen, $\mathrm{ab}$ dem 8. Trächtigkeitsmonat setzen dann Wachstum sowie Mineralisierung der Knochen verstärkt ein und verlaufen dann parallel zur Gewichtsentwicklung (MEYER, 1996), so dass das Fohlen als Nestflüchter zum Zeitpunkt seiner Geburt über ein bereits weit entwickeltes Skelett verfügt.

Bei der Knochenentwicklung (Ossifikation) unterscheidet man die desmale und die chondrale Ossifikation. Bei der desmalen oder direkten Knochenbildung erfolgt die Knochenbildung direkt, ohne knorpeligen Vorläufer aus mesenchymalem Bindegewebe. Aus den Mesenchymzellen bilden sich Osteoblasten, von denen aus die Knochenbildung erfolgt. Mit Hilfe der Osteoklasten erhalten die gebildeten Knochen schließlich ihre definitive Gestalt (MICHEL, 1983). Das Knochengewebe geht, wie alle anderen Binde- und Stützgewebe, aus dem Mesenchym hervor. Durch die Ausbildung der verschiedenen Formen von Bindegewebsfasern wandelt sich das Mesenchym unter gleichzeitiger Umformung der Zellen in die verschiedenen Arten des Bindegewebes um. Durch die Differenzierung der verschiedenen Fasern in der Grundsubstanz entstehen daraus die einzelnen Knorpelarten. Das Knorpelgewebe wird dann durch Knochen ersetzt, daher wird diese Art der Knochenbildung als indirekte oder chondrale Ossifikation bezeichnet Diese Ossifikationsart ist für das Breitenwachstum des Knochens verantwortlich. Bei der chondralen Ossifikation unterscheidet man weiterhin noch zwischen der enchondralen und der perichondralen Ossifikation. Die enchondrale Ossifikation erfolgt im Inneren des Knorpels, oder aber auch vom Perichondrium aus. Die Verknöcherung beginnt enchondral mit dem Auftreten von Verkalkunks-oder-Ossifikationszentren im Bereich der Gelenkenden (Epiphysen) oder kurzen Knochen. Es kommt zur Einlagerung von Kalksalzen an bestimmten Stellen des Knorpels und zur Vergrößerung der Zellen. Zur gleichen Zeit setzt die perichondrale Ossifikation ein, wodurch bei den Röhrenknochen eine den Knorpel umgebende Knochenmanschette entsteht. Nach Bildung des Knochens wird die Knorpelhaut (Perichondrum) zur Knochenhaut (Periost), so erfolgt das Breitenwachstum des Knochens. 
Das Längenwachstum des Röhrenknochens erfolgt in der knorpeligen Epiphysenfugenscheibe (Metaphyse), zwischen Diaphyse (Mittelstück des Knochens) und-Epiphyse (Endstück). Hier kommt es durch Chondrozyten (knorpelbildende Zellen) an einem Ende des sich entwickelnden Knochens zu einer ständigen Knorpelvermehrung, während am anderen Ende Knorpel abgebaut wird und so die Knochenbildung erfolgt.

Zum Zeitpunkt der Geburt des Fohlens ist ein Teil der Knochen schon völlig verknöchert, nur an einigen Epiphysenfugen erfolgt noch ein Längenwachstum der Knochen. Dieser Prozess dauert bis zum Alter von drei Jahren an, es erfolgt der Epiphysenschluss, das Wachstum des Pferdes ist damit abgeschlossen (HERTSCH und KROLL, 1999).

\subsubsection{Störungen der Knochenentwicklung/ Skelettreifung}

Verschiedene Faktoren können $\mathrm{zu}$ einer mangel- oder fehlerhaften Knochenentwicklung des Fohlens führen. Die Epiphysenfuge als Ort des Knochenwachstums besteht aus sehr stoffwechselaktivem Knorpel. Diese dort stattfindenden Stoffwechselvorgänge sind durch unterschiedliche Einflüsse leicht zu stören und zudem reagiert Knorpel, der aus weichem Gewebe besteht, auf besondere Belastungen sehr empfindlich. Knorpelgewebe ist somit für wachstumsbedingte Störungen in der Skelettreife prädestiniert. Dies ist der Grund, dass sich hier verschiedene entwicklungs- und wachstumsbedingte Erkrankungen lokalisieren (HERTSCH und KROLL, 1999), die man unter dem Begriff Developmental Orthopaedic Diseases (DOD) zusammenfasst. $\mathrm{Zu}$ diesem Komplex werden eine Reihe von Veränderungen und Erkrankungen gezählt, wie die Epiphysitis, die Verformung von Gliedmaßenknochen, Fehlstellungen der Gliedmaßen, Sehnenkontrakturen, das Wobbler-Syndrom, und die Osteochondrose. Mc ILWRAITH (1986) erklärt die Osteochondrose innerhalb des DOD Komplexes zur bedeutendsten entwicklungsbedingten Störung. 


\subsubsection{Störungen der Knochenentwicklung im Rahmen der OC}

Im Rahmen der Osteochondrose kommt es zu einer gestörten Differenzierung des wachsenden Knorpels. Anhand des Entstehungszeitpunktes wird die Osteochondrose als Erkrankung des jungen, wachsenden Tieres angesehen. Bei einer regulär ablaufenden enchondralen Ossifikation erfolgt die Versorgung der knorpelbildenden Zellen, der Chondrozyten, durch Diffusion, wobei der innere Fluss der Grundsubstanz durch Kompression und Dekompression, die bei Belastung oder Entlastung während der Bewegung entstehen, eine wesentliche Rolle für die Ernährung der Chondrozyten spielt (DÄMMRICH, 1985). Es kommt zu einer Pumpbewegung der Nährstoffe $\mathrm{zu}$ den Chondrozyten und somit $\mathrm{zu}$ einer ausreichenden Nährstoffversorgung des wachsenden Knorpels. Störungen bei der enchondralen Ossifikation führen zu einem stark verdickten Knorpelgewebe, das über die Grenzen des Diffusionsgradienten hinauswächst, so dass eine Ernährung dieses Gewebes und seiner Chondrozyten durch Synovialflüssigkeit nicht mehr gewährleistet ist (NIXON, 1993). Nicht nur im Stoffwechsel der Knochenmatrix kommt es $\mathrm{zu}$ Abweichungen, auch bei histologischen, biochemischen und immunohistochemischen Parametern konnten LILLICH et al. (1997) signifikante Unterschiede zwischen osteochondrotisch verändertem Knorpel und klinisch gesundem Knorpel feststellen. Aufgrund dieser Abweichungen ist die Ernährung der Chondrozyten nicht mehr gewährleistet, so dass es zu Nekrosen kleiner Blutgefäße kommen soll, die beim jungen Pferd in Knorpelkanälen verlaufen. Diese Knorpelnekrosen führen zu einer schuppenförmigen Abhebung des Gelenkknorpels (DÄMMRICH, 1985; SHINGLETON et al., 1997).

Das Krankheitsbild der Osteochondrose lässt sich röntgenologisch durch osteochondrotische Veränderungen in Form von Kontur /oder Strukturveränderungen des Knochens darstellen. Kommt es zur Osteochondrosis dissecans, spaltet sich das verdickte, unterernährte Knorpelgewebe, das zunehmend anfälliger für mechanische Belastungen wird, vom subchondralen Knochen ab, und es bildet sich ein frei im Gelenk beweglicher Gelenkkörper (DÄMMRICH, 1985; JEFFCOTT, 1991). 


\subsubsection{Störungen der Knochenentwicklung im Rahmen der OCD}

Die bei der Ostechondrosis dissecans vorliegenden losgelösten Knorpelstücke ossifizieren in der Regel sekundär, wodurch osteochondrale Fragmente entstehen, die eine weitere Schädigung des Gelenks verursachen können. Unter Einbeziehung der Gelenkoberfläche lässt sich so ein Chip nachweisen (SAMY, 1977; HERTSCH, 2000). Die Dissekate verbleiben entweder in der Nähe ihres Ursprungs, oder sind in einer sich bildenden Gelenksaussackung, dem sogenannten Mausbett, $\mathrm{zu}$ finden (McILWRAITH, 1989). Die Fragmente können Kirschkern- bis Linsengröße annehmen, wobei die tatsächliche Größe nur schwer geschätzt werden kann, da sich röntgenologisch nur der ossifizierte Kern darstellen lässt (HOPPE, 1984; STASHAK, 1989; HERTSCH, 1991). Die losgelösten Knorpelstücke können vor ihrer Ossifikation auch im Gelenk zerrieben werden, so dass häufig nur das Vorhandensein eines Mausbetts die Herkunft einer isolierten Verschattung andeutet. Generell erweist sich der radiologische Nachweis der Osteochondrosis dissecans im knorpeligen Frühstadium als problematisch, da sich knorpelige Strukturen im Röntgenbild nicht darstellen lassen (JEFFCOTT, 1993).

\subsection{Krankheitsverlauf/Klinische Symptome}

Nach ZELLER et al. (1978) lassen sich die beim Menschen beobachteten Stadien der Osteochondrosis dissecans auch auf das Pferd übertragen:

A) Latenzphase, Schlummerstadium des Sequesters in seinem Bett. Häufig zufällige Entdeckung

B) Stadium der Dissekation, Lahmheit durch Gelenkschmerzen bei einsetzender Abstoßung, vorzeitige Ermüdbarkeit, Gelenkschwellung

C) Stadium des freien Körpers mit Einklemmungserscheinungen und Ergüssen

D) Sekundäre Arthrosis deforma, verbunden mit Weichteilverdickungen durch eine chronische Synovitis

Auch HOFMANN und SCHÖNBAUER (1982) beschreiben das Frühstadium der Krankheit als eine nur geringfügig vorhandene Lahmheit mit gering ausgeprägter Umfangsvermehrung und Schmerzhaftigkeit des Gelenks. 
STASHAK (1989) und HERTSCH (2001) zählen in Kombination mit röntgenologischen Befunden zu den klinischen Symptomen weiterhin:

1) Lahmheit

2) Positiver Ausfall einer Provokationsprobe

3) Gelenkerguss

Jedes dieser Symptome kann trotz positivem röntgenologischen Befund fehlen, oder in unterschiedlichem Ausmaß in Erscheinung treten. Erst Training und reitsportliche Nutzung bewirken in der Regel eine deutlichere Manifestation der Symptome aus klinischer und radiologischer Sicht (HOFMANN und SCHÖNBAUER, 1982). ZELLER et al. (1978) vermuten, dass ca. 30\% der betroffenen Pferde an einer sogenannten „schlummernder Osteochondrose“ leiden, die zufällig anhand von Röntgenbildern oder durch intensives Training mit möglicher daraus folgender Lahmheit entdeckt wird.

\subsection{Diagnose der OC}

\subsubsection{Diagnose der OC anhand von Röntgenbildern}

Beim derzeitigen Stand der Diagnostik von Gelenkserkrankungen erfolgt die Diagnose der OC anhand einer röntgenologischen Untersuchung des betroffenen Gelenks.

Röntgenuntersuchungen stellen neben der klinischen Untersuchung ein wesentliches Hilfsmittel bei der Diagnose von Gliedmaßenerkrankungen dar (WINTER, 1995). Aufgrund der positiven Beziehung zwischen röntgenologischer Diagnose und der klinischen Bewertung eignen sich Röntgenbilder zur Einschätzung einer möglichen späteren Leistungsbeeinträchtigung (DIK et al., 1978). Anhand seiner Studie stellte HOPPE (1983) fest, dass die anhand der Röntgenbilder geschätzte Größe von freien Fragmenten durch die bei Operationen oder Autopsien gefundenen Chips bestätigt wurde, so dass anhand von Röntgenbildern getroffene Diagnosen als verlässlich angesehen werden können. 
Um die im Rahmen einer röntgenologischen Untersuchung erhobenen Röntgenbefunde eindeutig zuordnen und klassifizieren zu können, wurde 1993 ein Vierstufenschema von der Europäischen Röntgenkommission entwickelt (Tabelle 2).

Tabelle 2: Einteilungsschema der erhobenen Röntgenbefunde nach der europäischen Röntgenkommission (1993)
Kategorie I Röntgenologisch ohne besonderen Befund und Befunde, die als unbedeutend eingestuft werden.

Kategorie II Röntgenologische Befunde, die gering von der Norm abweichen, deren klinische Bedeutung unklar, unsicher oder unbekannt ist.

Kategorie III Röntgenologische Befunde, die erheblich von der Norm abweichen, bei denen eine negative Leistungsbeeinflussung jedoch ungewiss ist.

Kategorie IV Röntgenologische Befunde, die mit sehr großer Wahrscheinlichkeit zu einer negativen Leistungsbeeinflussung führen.

Das Spektrum dieses Bewertungsschemas reicht von „unbedeutend“ (Kategorie I) bis hin zur „deutlichen“ Abweichung (Kategorie IV) vom Normalzustand mit vorausgesetzter negativer Leistungsbeeinflussung.

Weitere Methoden zur Feststellung von Knochenqualität und Gliedmaßenveränderungen sind die Absorptionsphotometrie und die Ultraschall Messung (JEFFCOTT et al., 1986). Die Absorptionsphotometrie bietet die Möglichkeit einer Knochenmineralgehaltsmessung. Mit Hilfe des Ultraschalls können Knochenfestigkeit und -Elastizität gemessen werden. Beide Methoden geben genaue Auskünfte über den Reifegrad des Skeletts und können Krankheits- und Lahmheitseffekte auf die Knochen dokumentieren. 
Weiterhin bieten diese beiden Methoden die Erforschung der Effekte verschiedener Einflussfaktoren wie Immobilisierung, Bewegung und Fütterung auf die Knochenqualität beim lebenden Pferd.

Die Möglichkeit der OC-Diagnose anhand biologischer Parameter wie Knochenmarker wird unterschiedlich bewertet. OEXMANN (2004) stellte diese Methode als weitgehend abgesichert dar, während BILLINGHURST et al. (2005) noch weiteren Forschungsbedarf sehen, bis die Diagnose der OC anhand dieser Methode zur Verfügung stehen wird. Über molekulargenetische Methoden zur Diagnose von Osteochondrose wurde bisher nur beim Schwein berichtet (ANDERSSON-EKLUND, 2000), hier wurde ein genetischer Marker für diese Erkrankung entdeckt. Auch beim Pferd würde die Entdeckung genetischer Marker für die Osteochondrose möglicherweise zukünftig die Einbeziehung genetischer Informationen für die Selektion gegen OC möglich machen (RICARD, 2004).

\subsubsection{Diagnose der OC durch molekulargenetische Analysen}

Die Ergebnisse molekulargenetischer Studien an Nutztieren haben in den letzten Jahren einen Einblick in das Genom der Tiere ermöglicht und somit neue Perspektiven in der Tierzucht eröffnet. Genorte, die mit der Leistungsausprägung verschiedener Merkmale in Verbindung gebracht werden, sogenannte Quantitative Trait Loci (QTL) konnten identifiziert werden und tragen $\mathrm{zu}$ einem erhöhten Zuchtfortschritt bei (GUERIN, 2002).

Um die molekulargenetische Aufklärung genetisch beeinflusster Krankheiten beim Pferd voranzutreiben, ist das Vorhandensein einer „Genkarte“ unerlässlich. Beim Pferd wurde im Vergleich zum Rind oder zum Schwein erst vor relativ kurzer Zeit mit der Kartierung von Genen begonnen. Aus diesem Grund sind die Genkarten beim Pferd erst in einer frühen Entwicklungsstudie. In diesem Zusammenhang sind Studien von CAETANO et al. (1999) sowie SWINBURNE et al. (2002) zu nennen.

In der Pferdezucht vorhandene Gendefekte, für die bereits ein direkter Gentest entwickelt werden konnte, sind die Severe Combined Immunodeficiency (SCID), und die Hyperkalaemic Periodic Paralysis (HYPP) (COLLINDER und RASMUSSON, 2000). 
SCID ist eine autosomal rezessiv vererbte Immunschwäche. Man schätzt, dass etwa $10 \%$ der arabischen und part-bred arabischen Pferde in den USA Träger dieser Erkrankung sind (COLLINDER und RASMUSSON, 2000). HYPP wird im kodominant autosomalen Erbgang übertragen (COLLINDER und RASMUSSON, 2000) und wurde vermehrt in Quarter Horses beobachtet. Die Erkrankung äußert sich durch muskelphysiologische Störungen.

Die Beteiligung einer genetischen Komponente an der Entstehung der Osteochondrose wird allgemein angenommen (PHILLIPSSON et al., 1993; WINTER, 1996; SCHOBER, 2003). Die Gene, die eine Rolle bei der Entstehung der OC beim Pferd spielen, sind jedoch noch nicht charakterisiert (LÖHRING, 2003). Ist kein direkter Gentest vorhanden, können Marker verwendet werden, um Wahrscheinlichkeitsaussagen über das Vorhandensein bestimmter Allele zu treffen (REINSCH, 2002). Marker können als Markierung oder Hinweis auf phänotypischer oder genotypischer Ebene angesehen werden, die auf das Vorhandensein eines Gens hindeuten können. LÖHRING (2003) beschäftigte sich mit der Entwicklung eines Markersets, das bei der Identifizierung eines QTL für die Osteochondrose helfen soll. Derzeit wird an der Entwicklung weiterer ergänzender Marker gearbeitet, die im Rahmen der QTL-Suche nach Osteochondrosis beeinflussenden Genorten eingesetzt werden sollen (BÖNECKER, 2004).

\subsection{Einflussfaktoren auf die Entstehung der OC /OCD}

Die Osteochondrose wird als Erkrankung des jungen, wachsenden Fohlens angesehen. Der Knorpel ist zu diesem Zeitpunkt in einer sehr wachstums- und stoffwechselintensiven Phase und somit empfindlich gegenüber endogenen Einflüssen. Die folgende Abbildung 1 soll die möglichen Einflüsse dieser Faktoren auf den wachsenden Knorpel verdeutlichen. 


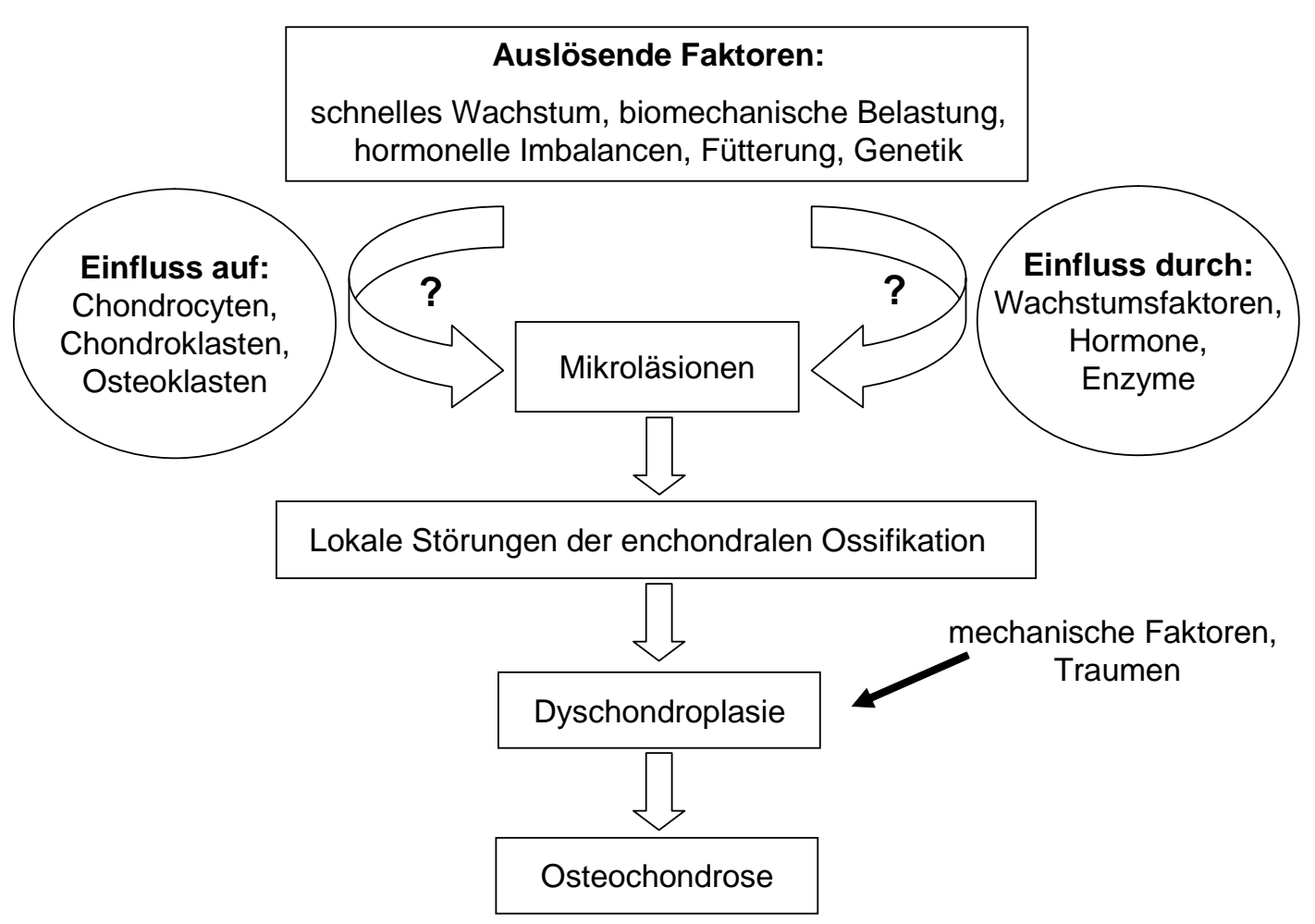

Abbildung 1: Einflussfaktoren auf die Entstehung der Osteochondrose (modifiziert nach JEFFCOTT, 2004)

Als weiterer auslösender Faktor für die Entstehung der Erkrankung wird eine mangelnde Durchblutung des wachsenden Knorpels gesehen, dieser Ansatz wird in der Literatur unterschiedlich bewertet. EKMAN und CARLSON (1998) sehen Durchblutungsstörungen im enchondralen Knorpelgewebe als ursächlichen Grund für die Entstehung der Osteochondrose an. Aufgrund der Durchblutungsstörungen komme es zu veränderten und hochempfindlichen Knorpelgewebszonen, die sich bei mechanischer Belastung vom Knochen abspalten und $\mathrm{zu}$ freien Gelenkskörpern werden können. Ihrer Meinung nach sollte die Erforschung der Ursache für das Auftreten dieser Durchblutungsstörungen weitere Studien nach sich ziehen. SAMY (1977) hingegen sieht aufgrund der Ergebnisse seiner arteriographischen Untersuchungen keinen Zusammenhang zwischen der Entstehung der Osteochondrose aufgrund von mangelhaft durchblutetem Knorpelgewebe. Auch HERTSCH (1981) konnte den Einfluss der Gefäßversorgung auf die Entstehung von osteochondralen Veränderungen nicht nachweisen. 
Die initialen Läsionen der Osteochondrose werden als Folge einer gestörten enchondralen Ossifikation angesehen, die wirkliche Ursache für die Entstehung der Erkrankung ist jedoch noch nicht bekannt. Es wird ein multifaktorielles Geschehen angenommen, in dem Vererbung, Wachstum, Fütterung, mineralische Imbalancen, endokrine Dysfunktionen und biomechanische Traumen eine Rolle spielen (JEFFCOTT, 1991). Diese aufgeführten Einflussfaktoren und ihre mögliche Beteiligung an der Entstehung der Osteochondrose waren das Thema verschiedener Studien, über die im Folgenden ein Überblick gegeben werden soll.

\subsubsection{Fütterung}

Der Ätiologie der Gelenkerkrankung Osteochondrosis dissecans wird in der Literatur ein multifaktorieller Hintergrund zugeschrieben. Eine besonders große Bedeutung hat in diesem Zusammenhang der Bereich der Pferdeernährung. Begünstigende Faktoren für die Entstehung der Erkrankung scheinen vor allem eine über dem Bedarf liegende Energie- und Proteinversorgung beim jungen wachsenden Pferd mit möglichen metabolischen und endokrinen Entgleisungen sowie Imbalancen bei der Mineralstoffversorgung zu sein (JEFFCOTT, 1997; WINKELSETT, 2003).

Wie in verschiedenen wissenschaftlichen Versuchen nachgewiesen werden konnte, scheint eine über dem Bedarf liegende Versorgung mit Energie beim jungen, wachsenden Pferd maßgeblich zur Entstehung von OCD beizutragen. GLADE und BELLING (1984) konnten durch Fütterung von 130\% der NRC Empfehlung für Energie Dysplasien und Osteochondrose ähnliche Läsionen hervorrufen.

In einer Reihe von kontrollierten Experimenten zeigte SAVAGE (1991), dass hohe Energiegaben (128\% über NRC Empfehlung) osteochondrotische Veränderungen bei Fohlen erzeugten. Fohlen, die in diesem Versuch mit 100\% der NRC Empfehlung für Energie gefüttert wurden, zeigten hingegen keine Veränderungen. Auch LEWIS (1998) ist der Ansicht, dass eine übermäßige Energiezufuhr eine der wichtigsten Ursachen für eine gestörte enchondrale Ossifikation ist. Er weist aber auch daraufhin, dass eine einem Energieüberschuss zugeschriebene gestörte enchondrale Ossifikation auch auf eine der damit erhöhten Wachstumsrate $\mathrm{zu}$ geringe Versorgung mit Phosphor, Kalzium oder anderen Mineralstoffen zurückzuführen sein kann. 
Zur Verhütung von Schäden am Skelett müsse die Ration der tatsächlichen Wachstumsrate angepasst werden. BORCHERS (2002) konnte in ihren Untersuchungen jedoch keinen Zusammenhang zwischen der Rohprotein- und Energieversorgung der Stute und dem Vorkommen der Osteochondrose beim Fohlen im Hinblick auf die Körpergewichts- und Körpergrößenentwicklung des Fohlens feststellen. Allerdings konnte hier die tatsächlich aufgenommene Futtermenge der Fohlen nicht genau kalkuliert werden, dieser Unsicherheitsfaktor hat sich möglicherweise auf die statistische Auswertung ausgewirkt. Weiterhin wird ein möglicher Zusammenhang zwischen energiebetonter Fütterung und der Entstehung von Hyperglykämie bzw. Hyperinsulinämie in der Literatur diskutiert. Hohe Insulinkonzentrationen, die bis zu vier Stunden nach der Fütterung von vor allem kohlenhydratreichem Futter (hohe Maisanteile) gemessen werden konnten (RALSTON, 2004), werden sowohl durch ihre direkten als auch ihre indirekten endokrinologischen Auswirkungen mit Veränderungen am Knorpel in Verbindung gebracht (JEFFCOTT und HENSON, 1998)

Der Einfluss von übermäßiger Proteinversorgung auf eine gestörte enchondrale Ossifikation wird in der Literatur unterschiedlich bewertet. SAVAGE et al. (1993) verglichen den Einfluss von übermäßiger Energie- und übermäßiger Proteinfütterung auf die Entstehung von osteochondralen Veränderungen. Hier zeigte sich bei Tieren, die mit 129\% der NRC Empfehlung für Energie gefüttert wurden, signifikant häufiger klinische und radiographische Anzeichen von Osteochondrose, als bei der Kontrollgruppe. Das Auftreten von Veränderungen bei den Fohlen mit übermäßig hoher Proteinversorgung (126\% der NRC Empfehlungen für Protein) war hingegen nicht signifikant höher als bei der entsprechenden Kontrollgruppe. Auch RALSTON (1994) sieht keinen Zusammenhang zwischen hohen Gaben von Protein und der Entstehung von orthopädischen Entwicklungsstörungen (DOD). In einer Untersuchung verglichen SCHRYVER et al. (1987) den Effekt unterschiedlich hoher Proteinfütterung auf die Wachstumsrate von 24 Fohlen. Es zeigte sich, dass eine niedrige (9\%) Proteingabe einen wachstumsdepressiven Einfluss hatte. Das Wachstum der Fohlen, die mit einer mittleren (14\%, NRC Empfehlung) und einer hohen (20\%) Proteingabe versorgt wurden, zeigte hingegen keinen Unterschied. THOMPSON et al. (1988) fanden hingegen Knochenveränderungen bei einer übermäßigen Fütterung von Protein (275\%) und Energie (150\%). 
Einen möglichen Erklärungsansatz hierfür liefern LEWIS (1998) sowie JEFFCOTT und SAVAGE (1996) mit der Aussage, dass eine übermäßige Proteinaufnahme die renale Ausscheidung von Kalzium erhöhen kann, dadurch ein sekundärer Kalziummangel entsteht, und die enchondrale Ossifikation beeinträchtigt wird. AHTILA und SAASTAMOINEN (2004) untersuchten den Einfluss der Ernährung auf die Wachstumsrate bei Absetzern. Sie stellten fest, dass eine erhöhte Fütterung von Energie zu einem erhöhten Wachstum führt. Eine übermäßige Fütterung von Protein führte in diesem Versuch hingegen zu einem Rückgang im Wachstum. Die Autoren führen diesen Umstand auf den möglichen erhöhten Kalorienverbrauch zurück, der beim Abbau von Aminosäuren benötigt wird. Als Folge einer Unterversorgung mit Energie stellten die Autoren ein kompensatorisches Wachstum der Fohlen fest.

Auch Störungen im Mineralhaushalt scheinen bei der Pathogenese von Osteochondrose eine Rolle zu spielen (JEFFCOTT, 1991). Eine ausreichende Versorgung mit den Mengenelementen Kalzium und Phosphor ist notwendig für die enchondrale Ossifikation (LEWIS, 1998). Ein Kalzium- bzw. Phosphormangel äußert sich durch eine reduzierte Kalzifizierung des Knochens, Lahmheiten oder Knochendeformationen. THOMPSON et al. (1988) wiesen in ihren Untersuchungen bei mangelernährten Fohlen Knochenschäden und geringe Knochenmineralgehalte nach. SAVAGE (1991) fand kein vermehrtes Auftreten von Osteochondrose bei erhöhter Gabe von Kalzium. Bei erhöhter Kalzium Gabe in Kombination mit hohen Energiegaben wurde hingegen ein vermehrtes Auftreten von Osteochondrose festgestellt. Auch bei hohen Phosphor- und Energiegaben konnten in diesem Fütterungsversuch makroskopisch und histologisch nachweisbare osteochondrale Läsionen nachgewiesen werden. Im Überschuss aufgenommener Phosphor und Oxalate binden Kalzium im Darm und reduzieren so die Absorptionsrate. Trotz ausreichender Kalziumversorgung kann es dann durch die verminderte Absorption zu einem Kalziummangel kommen. Im umgekehrten Fall hat ein erhöhter Kalziumgehalt wenig Einfluss auf die Absorption des Phosphors, da Kalzium hauptsächlich im Dünndarm, Phosphor aber sowohl im Dünn- als auch im Dickdarm absorbiert wird (LEWIS, 1998). Durch eine Überversorgung mit Kalzium kann allerdings die Absorption einiger Spurenelemente im Darm beeinträchtigt werden (LEWIS, 1998). Dies gilt vor allem für Zink, Mangan und Eisen. 
Liegen die in der Ration enthaltenen Mengen dieser Spurenelemente in der unteren Grenze der Bedarfsnorm, kann hier durch eine unkontrollierte Gabe von kalziumhaltigen Mineralfuttermitteln eine Mangelsituation entstehen.

Weiterhin haben die Spurenelemente Zink, Kupfer sowie eventuell auch Mangan möglicherweise ebenfalls eine Bedeutung bei der Entstehung von Osteochondrose (LEWIS, 1998). Kupfer wird für die Elastinsynthese und die Stabilisierung der kollagenen Fasern im Knochen benötigt. Kupfer ist außerdem ein Bestandteil des Enzyms Lysil Oxidase, welches für die Verknüpfung von Kollagenfasern benötigt wird (VAN WEEREN, 2003). BRIDGES und HARRIS (1988) berichten von kupfermangelversorgten Fohlen, in deren Blut extrem hohe Mengen des Enzyms Collagenase $\mathrm{zu}$ finden war. Dieses Enzym verringert die Verknüpfung von Kollagenfasern und schafft damit die Prädisposition für biomechanische Traumen und weitere Schäden. HURTIG et al. (1990) verglichen Fohlen mit erhöhter und verminderter Kupfer Gabe, wobei ein stark vermehrtes Auftreten von osteochondralen Läsionen bei den Fohlen mit geringem Kupfergehalt im Futter verzeichnet wurde. Die im Rahmen ihrer Studie untersuchten Fohlen bezeichnete GRANEL (2003) als kupferunterversorgt. Dies lässt sich auf den niedrigen Kupfergehalt der Stutenmilch zurückführen, aber eine mögliche Verbindung zu entwicklungsbedingten Störungen wurde hier nicht ausgeschlossen. VAN WEEREN (2004) weist darauf hin, dass Kupfer möglicherweise nur einen geringen Einfluss auf die Pathogenese von Osteochondrose hat, aber bei möglichen natürlichen Reparaturprozessen der Läsionen eine Rolle spielt. Eine übermäßige Zinkaufnahme beeinträchtigt die Absorption von Kalzium und Kupfer, sodass Störungen der osteochondralen Ossifikation in diesem Zusammenhang als Folge eines sekundären Kalzium- oder Kupfermangels auftreten können. Bei übermäßiger Zinkaufnahme wurden bei Pferden außerdem verschiedene Erkrankungen der Knochen und Gelenke wie Epiphysitis und chronische Gelenkschwellungen festgestellt (LEWIS, 1998). Zink spielt während des Skelettwachstums eine wichtige Rolle, wobei die Effekte einer Zinkmangelversorgung noch nicht ausreichend dokumentiert sind (RALSTON, 1997; LEWIS, 1998). 


\subsubsection{Wachstum}

Wachstum wird definiert als ein Überwiegen von anabolem (Stoffansatz) gegenüber katabolem Stoffumsatz Prozessen (KÜNZI und STRANZIGER, 1993). Wachstum ist gekennzeichnet durch eine Größenänderung, im Allgemeinen eine Zunahme (MICHEL, 1983). Auf der Zellebene bedeutet Wachstum eine Zellvermehrung, Zellvergrößerung und Zelldifferenzierung. Auf der Ebene des Tieres bedeutet Wachstum eine Veränderung der Größen- und Gewichtszunahme, der Körperproportionen und der Körperzusammensetzung. Wachstum hat einen allometrischen Verlauf, das bedeutet das Wachstum der Körperorgane verläuft unterschiedlich im Vergleich zur Wachstumsgeschwindigkeit des gesamten Organismus. Die Reihenfolge der sich entwickelnden Körperteile ist immer gleich, das Zentralnervensystem entwickelt sich am frühesten, es folgen die Knochen, die Muskeln und am Schluss das Körperfett (KÜNZI und STRANZIGER, 1993). Man unterteilt das Wachstum üblicherweise in das pränatale und das postnatale Wachstum. Während des pränatalen Wachstums kommt es zur Zelldifferenzierung und zur Organentwicklung. Das postnatale Wachstum untergliedert sich in drei Phasen: das Jugendstadium, das Reifestadium und das Stadium des Alterns. Im Jugendstadium erfolgt das Höhen- und Längenwachstum, die Organausbildung, das Muskelwachstum und in diesem Zusammenhang besonders wichtig, die Ossifikation. Im Reifezustand werden die Geschlechtsreife und die maximale Größe erlangt. In diesem Stadium liegt ein Gleichgewicht zwischen katabolen und anabolen Prozessen vor. Das Altern ist schließlich gekennzeichnet durch eine verringerte Proteinsyntheseaktivität (MICHEL, 1983).

Das Wachstum allgemein und im speziellen beim Fohlen wird durch verschiedene Faktoren beeinflusst, als wichtigste Faktoren nennen HERTSCH und KROLL (1999):

1) Genügende Zufuhr eines qualitativ wertvollen Aufbaumaterials

2) Geregelter Ablauf aller Stoffwechselvorgänge

3) Altersentsprechende Regulation durch verschiedene Hormone

4) Altersentsprechende Wachstumspotenz und Ansprechbarkeit der Zielgewebe durch die Hormone

5) Integration durch genetische Faktoren 
Die hormonelle Regelung des Wachstums erfolgt über das endokrine System, von besonderer Bedeutung sind in diesem Zusammenhang die „Insulin Like Growth Factors (IGFs) sowie die Thyroid Hormone (Thyroxin, T4, und Tri-Jodothyronin, T3) (WINKELSETT, 2003).

Der Wachstumsverlauf von Fohlen und vor allem die Parameter Körpergröße und Körpergewicht waren bereits Gegenstand intensiver Studien. Anhand dessen hat man festgestellt, dass die meisten Pferderassen bereits bei der Geburt 61-65\% der späteren Endgröße erreicht haben. Im weiteren Wachstumsverlauf werden mit sechs Monaten 83\%, mit 12 Monaten 90\% und mit 18 Monaten etwa 95\% der genetisch festgelegten Endgröße erreicht (JACKSON et al., 1993). Die intensivste Wachstumsphase findet dabei innerhalb der ersten drei Monate statt. Nach dem Absetzen setzt sich die Wachstumsgeschwindigkeit weiter fort und endet mit dem ersten Lebensjahr. Eine dritte Phase intensiven Wachstums kann man zum Teil auch während der Pubertät feststellen (JELAN et al., 1996). Im Vergleich zur Körpergröße unterliegt das Körpergewicht einem weniger konstanten Verlauf (LEWIS, 1995), da es stärker durch äußere Umweltfaktoren wie z.B. Fütterung in seiner Ausprägung und Entwicklung beeinflussbar ist. Zum Zeitpunkt seiner Geburt hat das Fohlen ca. $10 \%$ seines Endgewichtes erreicht (MEYER, 1996). Während des weiteren Wachstums werden im sechsten Lebensmonat etwa 45-50\%, im 12.Lebensmonat 65 bis $70 \%$ und im 18. Lebensmonat ungefähr 85\% des Endgewichts erlangt (LEWIS, 1995). Die Obergrenze der Wachstumskapazität des Fohlens ist durch genetische Faktoren festgelegt. Die untere Grenze des Wachstumsvorgangs wird hingegen durch umweltbedingte Faktoren beeinflusst. Niedrige Umgebungstemperaturen haben hier keinen Einfluss, solange eine ausreichende und regelmäßige Fütterung erfolgt (CYMBALEK und CHRISTISON, 1989). Wachstumsstörungen konnten hingegen festgestellt werden durch äußere Einflüsse wie z. B. eine zu geringe Futterzufuhr, Parasitenbefall und chronische Verdauungsstörungen (BRAMLAGE, 1986).

WILLARD et al. (2004) untersuchten den Einfluss des Alters der Mutterstute sowie den Umstand, dass Fohlen von Ammen und nicht von ihren Müttern aufgezogen wurden, auf die Wachstumsrate von Vollblutfohlen bis zum Altern von neuen Monaten. 
Die Ergebnisse zeigten, dass ältere Zuchtstuten (> 20 Jahre) im Durchschnitt um 4kg leichtere Fohlen $(\mathrm{p}<0,1)$ erzeugten als jüngere Stuten (19 Jahre oder jünger). Die Fohlen der älteren Stuten zeigten im Gegensatz zu den anderen Fohlen aber eine signifikant höhere Wachstumsrate $(0,07 \mathrm{~kg} / \mathrm{Tag})$. Fohlen, die von Ammen aufgezogen wurden zeigten ein signifikant schnelleres Wachstum $(0,036 \mathrm{~kg}$ tz/Tag) als Fohlen, die von ihrer Mutter aufgezogen wurden. Die Ammenstuten, (reinrassige Tenesse Walking Horses oder Kreuzungsprodukte dieser Rasse mit anderen Rassen, die ein ähnliches Gewicht aufwiesen wie die Vollblutstuten) wurden aber im Gegensatz zu den Vollblutstuten nicht aufgrund von Rennbahnleistung selektiert, sondern aufgrund ihrer Milchproduktion und ihrer mütterlichen Verhaltensweisen.

Hengstfohlen haben bei der Geburt ein deutlich höheres Gewicht und einen höheren Widerrist als Stutfohlen. Inwieweit sich dieser Geschlechtsdimorphismus auf die Ausprägung von Osteochondrose niederschlägt, wird in der Literatur kontrovers diskutiert. SAMY et al. (1977) konnten in ihren Untersuchungen einen deutlichen Effekt des Geschlechts auf das Auftreten von Osteochondrose nachweisen. DÄMMRICH (1985) kommt in seinen Ausführungen zum gleichen Ergebnis. HOPPE (1987) konnte hingegen in seiner Langzeitbeobachtung über einen Zeitraum von sechs Jahren keine signifikanten statistischen Unterschiede zwischen den Geschlechtern feststellen. Auch in einer holländischen Langzeitstudie konnte kein Zusammenhang zwischen dem Geschlecht der Fohlen und dem Auftreten von OC festgestellt werden (VAN WEEREN et al., 1999).

Mehrere Exterieurmerkmale gelten als prädisponierende Faktoren für das Auftreten der Osteochondrosis, so wird in der Literatur vielfach ein Zusammenhang gesehen zwischen der Ausprägung von osteochondrotischen Läsionen und schnellem Wachstum bei vor allem großrahmigen Pferden (STROMBERG, 1979; SANDGREN et al., 1993; RALSTON, 1997; LILLICH, 1997). DÄMMRICH (1985) sieht den Grund für diese Problematik vor allem in einer energie- und proteinreichen Fütterung, die ein schnelles Wachstum hervorrufe, durch die Skelettwachstum und Muskelentwicklung zwar beschleunigt würden, aber die dadurch größer dimensionierten, jedoch nicht belastungsfähigeren Knochen würden einer Überbelastung durch die verstärkt einwirkenden Muskelkräfte ausgesetzt. 
Auch HERTSCH und KROLL (1999) weisen auf bestehende Wechselwirkungen zwischen der stützenden Bedeutung des Skeletts einerseits und seiner begrenzten Belastbarkeit durch das wachsende Körpergewicht andererseits hin. Die Ergebnisse mehrer Studien unterstreichen die möglichen gesundheitlichen Probleme, die aus einem schnellen Wachstum entstehen können. SANDGREN (1993) untersuchte in seiner Studie den Einfluss des Wachstums auf Osteochondrose anhand von 77 Traberfohlen. Er stellte fest, dass die Fohlen mit Befunden im Sprunggelenk (10\%) einen größeren Röhrbeinumfang hatten, als die nicht betroffenen und außerdem einen höheren Widerrist hatten. Fohlen, bei denen osteochondrale Fragmente im Fesselgelenk festgestellt wurden (14\%) waren hingegen kleiner, leichter und hatten einen geringeren Röhrbeinumfang. Diese Erkenntnisse decken sich mit den Ergebnissen, zu denen WILKE (2003) anhand ihrer Untersuchungen in der Hannoverschen Warmblutpopulation gelangte. Auch PAGAN und JACKSON (1996) berichteten anhand ihrer Studien, dass Fohlen mit osteochondrotischen Veränderungen im Sprunggelenk schwerer waren als der Durchschnitt. VAN WEEREN et al. (1999) stellten im Hinblick auf das Körpergewicht signifikante Zusammenhänge mit der Entstehung von Osteochondrose im Kniegelenk, nicht aber im Sprunggelenk fest. JELAN et al. (1996) dagegen konnten dagegen innerhalb ihrer Untersuchungen hinsichtlich entwicklungsbedingter Wachstumsstörungen keine signifikanten Gewichts- und Größenunterschiede zwischen normalen und betroffenen Fohlen finden.

VAN WEEREN (2004) verdeutlichte in seinem Artikel, dass die Osteochondrose beim jungen Fohlen einen dynamischen Prozess darstellt. Im Verlauf dieses Prozesses können röntgenologisch sichtbare Defekte entstehen, die aber beim Erreichen eines bestimmten Alters auch wieder völlig verschwunden sein können (CARLSTEN et al., 1993; DIK et al., 1999). Die nach der Geburt rasch absinkende Knorpelmetabolismusrate macht es bei zunehmendem Alter immer unwahrscheinlicher, dass Defekte noch repariert werden können. Das bedeutet, dass Läsionen, die spät entstehen oder sehr groß sind mit einer größeren Wahrscheinlichkeit endgültig klinisch manifest sind (VAN WEEREN, 2004). 


\subsubsection{Bewegung / Mechanische Belastung}

Bewegung fördert die Entstehung eines gesunden und widerstandsfähigen Knorpels, ein Mangel an Bewegung führt zu Beeinträchtigungen der normalen Entwicklung des Gelenks. Ein bestimmtes Maß an Bewegung scheint eine essentielle Bedeutung für eine optimale funktionelle Adaption des Knorpels zu haben, die vor allem in den ersten fünf Lebensmonaten stattfindet. Ein Fehlen dieser Bewegung kann möglicherweise zum Ausbleiben der entsprechenden Adaption führen (BRAMA et al., 2002). Somit ist es dringend zu empfehlen, Fohlen so früh wie möglich und so lange wie möglich Freilauf auf der Koppel oder auf dem Paddock zu ermöglichen (RALSTON, 1996).

Der Einfluss von Bewegung auf die spätere Knochenentwicklung wurde bereits in mehreren Studien untersucht (JEFFCOTT et al., 1988). In ihrer Feldstudie kam WILKE (2003) zu dem Ergebnis, dass früh (vor dem 1. April) geborene Fohlen als Folge der mangelnden Bewegung aufgrund des frühen Geburtszeitpunktes deutlich häufiger einen OC Befall aufwiesen als die später geborenen Fohlen. Dieser Effekt wurde für das Fesselgelenk deutlicher nachgewiesen als für das Sprunggelenk. Somit konnte festgestellt werden, dass durch häufige und regelmäßige Bewegung der Fohlen die Frequenz von OC insgesamt deutlich gesenkt werden konnte. Eine Langzeitstudie in den Niederlanden verglich verschiedene Haltungs- und Bewegungsformen und deren Einfluss auf die Ausprägung von Osteochondrose miteinander. $\mathrm{Zu}$ diesem Zweck wurden 43 für Osteochondrose im Sprunggelenk genetisch prädisponierte Fohlen in verschiedene Haltungsgruppen aufgeteilt und über einen Zeitraum von fünf Monaten beobachtet Die Haltungsform der ersten Gruppe bestand aus reiner Boxenhaltung, die zweite Gruppe wurde auch in der Box gehalten, erhielt zusätzlich ein dosiertes, aber intensives Galopptraining. Die dritte Gruppe schließlich wurde permanent auf der Weide gehalten. Nach dem Absetzen wurden acht Fohlen aus jeder Gruppe zum Zweck der Knochenuntersuchung euthanasiert. Die verbleibenden Tiere wurden alle der gleichen Haltung sowie dem gleichen leichten Bewegungsregime unterzogen, bis sie dann im Alter von elf Monaten zu gleichen Zweck ebenfalls euthanasiert wurden. 
Die Ergebnisse der Knochenuntersuchungen zeigten, dass die Bewegung keinen signifikanten Einfluss auf die Zahl der Läsionen hatte, in der Gruppe der fünf Monate alten Fohlen zeigten die Tiere aus reiner Boxenhaltung jedoch deutlich ausgeprägtere Läsionen (VAN WEEREN et al., 1999). BARNEVELD et al. (1999a) stellten anhand des gleichen Datenmaterials fest, dass die Fohlen aus reiner Stallhaltung eine signifikant geringere Knochendichte aufwiesen, als die Fohlen mit Bewegungsregime und die Fohlen aus Weidehaltung. In einer weitergehenden Untersuchung am gleichen Datenmaterial untersuchten FIRTH et al. (1999) die Einflüsse von Alter, Bewegung und Wachstumsrate auf den Knochenmineralgehalt. Auch hier wurde ein positiver Einfluss der Bewegung auf die Entwicklung des Bewegungsapparates und der Skelettmuskulatur festgestellt. Die Fohlen mit einem erhöhten Grad an Bewegung wiesen eine höhere Anzahl der für die Durchbauung des Knochens wichtigen Knochenbälkchen auf. HOEKSTRA et al. (1999) verglichen in diesem Zusammenhang über einen Zeitraum von 140 Tagen verschiedene Knochenmineralgehaltparameter zwischen Pferden aus Boxenhaltung und Pferden, die auf der Weide gehalten wurden. Die Ergebnisse zeigten, dass eine Boxenhaltung bei Pferden, die noch in der Entwicklung stehen, zu einem geringeren Knochenmineralgehalt im Vergleich zu Pferden, die auf der Weide gehalten werden, führt.

HERTSCH (1992) weist auf die Bedeutung einer regelmäßigen und ruhigen Bewegung hin. Eine unangepasste Bewegung sei durch die unregelmäßige und kurzfristige Hochbelastung der Gliedmaßen zu vermeiden. Der Knorpel benötige 1015 Minuten, um seine volle Druckelastizität und Scherfähigkeit durch Wasserspeicherung zu erreichen. Solange müsse ein Pferd locker und ohne Belastung mindestens bewegt werden, bevor Leistungen mit höherer Belastung verlangt werden könnten. Oftmals toben Pferde aber genau in diesen ersten 10 Minuten des Freilaufens ihre aufgestaute Bewegungsenergie aus. Aus diesem Grund seien bereits bei jungen Pferden arthrothische Erscheinungen ein besonders häufiger röntgenologischer Befund. 


\subsubsection{Endokrinologie des Knochenstoffwechsels}

Hormonelle Regelfunktionen sowie verschiedene Parameter des Knochenstoffwechsels spielen unter Umständen eine entscheidende Rolle bei der Entstehung von Osteochondrose. Die Untersuchungen der letzten Jahre haben gezeigt, dass biochemische Knochenmarker ein geeignetes Instrument zur Überwachung des Knochenstoffwechselverlaufs darstellen (WINKELSETT, 2003; FIRTH, 1999; LENSING, 1998; PRICE et al, 1995).

Als Parameter des Knochenaufbaus können das Osteocalcin und das Typ-I Kollagen PICP angesehen werden. Osteocalcin ist ein für Knochengewebe spezifisches, nichtkollagenes Protein, dessen Synthetisierung in der Phase der Mineralisation erfolgt (CAMARDA et al., 1987). Es gilt daher als Marker der Osteoblastenfunktion, seine genaue Funktion ist jedoch noch ungeklärt, möglicherweise spielt es bei der Regulierung von Kalzifikationsprozessen eine Rolle (SEIBEL et al., 1993). Das Carboxyterminale Propeptid des Typ I Kollagens PICP ist eines der Propeptide, die 90\% der Knochensubstanz ausmachen. Es soll einen vorzeitigen Zusammenschluss $\mathrm{zu}$ Kollagenfibrillen verhindern und wird bei deren Bildung durch Endopeptdidasen abgespalten und in den Blutkreislauf abgegeben (LIESEGANG, 2000). Diese Propeptide können als quantitatives Maß der Kollagenneubildung angesehen werden, ein Teil der gemessenen Konzentrationen kann jedoch auch aus anderen Geweben stammen, sie sollten daher in ihrer Interpretation nicht überbewertet werden (LE PAGE et al., 2001).

Resorptive Vorgänge am Knochen können anhand der Teleopeptide des Typ I Kollagens, insbesondere des ICTP (Carboxyterminales Teleopeptid des Typ I Kollagen) untersucht werden. Es wird in der Phase der Knochenresorption durch Osteoklasten (knochenabbauende Zellen) in die Blutbahn abgegeben (LE PAGE et al., 2001).

Äußere Einflüsse wie das Körpergewicht und jahreszeitliche Schwankungen konnten als signifikante Einflüsse auf die Konzentration von Knochenmarkern im Blutserum nachgewiesen werden (LE PAGE et al., 1990; Price, et al, 2001). Ein deutlicher Abfall der genannten Parameter im Blutserum konnte außerdem bei zunehmendem Alter festgestellt werden (LE PAGE et al., 1990; PRICE et al., 2001). 
Dieser Abfall der Knochenparameter mit zunehmendem Alter deckt sich mit den Erkenntnissen von BRAMA et al. (2000). In ihren Untersuchungen stellten sie fest, dass das Knochengewebe, um gegenüber Belastungen eine gewisse Flexibilität zu entwickeln, eine biochemische Heterogenität benötigt, die sich aufgrund der Adaption an verschiedene Belastungszustände in den ersten fünf Lebensmonaten entwickelt. Diese Entwicklung in den ersten fünf Lebensmonaten post partum sei besonders wichtig, da zu diesem Zeitpunkt die Collagen-Turnover-Rate noch sehr hoch ist und somit eine gegenüber Belastungen stabile Collagenvernetzung des Knochens entstehen kann. Die Collagen-Turn-Over-Rate sinkt im Laufe des Wachstums immer weiter ab.

Auch Training, und Haltung haben einen Einfluss auf die Knochenmarkerkonzentration. HOEKSTRA et al. (1999) verglichen die Knochenmineralgehalte von Pferden aus Boxenhaltung und Pferden, die auf der Weide gehalten wurden über einen Zeitraum von 140 Tagen. Anschließend absolvierten Pferde aus beiden Haltungsgruppen ein 56tägiges Training. Die im Stall gehaltenen Pferde wiesen durchweg schlechtere Werte des Knochenmineralgehalts auf, als die Pferde in Weidehaltung. Die Ergebnisse zeigten, dass eine Stallhaltung bei in der Entwicklung stehenden Pferden einen Verlust an Mineralstoffgehalten im Knochen nach sich ziehen kann.

Weiterhin wird in der Literatur insbesondere auf das Risiko erhöhter Insulinkonzentrationen im Blut nach der Gabe stärkereicher Rationen hingewiesen (RALSTON, 1996). Auch JEFFCOTT und HENSON (1998) sehen hier ein erhöhtes Risiko für die Entstehung von Osteochondrose, da ein hoher Insulinspiegel im Blut, resultierend aus einer energiereichen Fütterung, die Chondrozyten Reifung beeinflussen und $\mathrm{zu}$ einem gestörten Matrix Metabolismus führen kann, dieser wiederum führt $\mathrm{zu}$ einer fehlerhaften Knochenmineralisierung und $\mathrm{zu}$ Knorpelläsionen, die als erste Anzeichen von Osteochondrose angesehen werden. GLADE et al. (1984) konnten in diesem Zusammenhang die vorübergehende Abnahme von T4 (Thyroxin) nach der Verfütterung einer energiereichen Ration parallel zu hohen Insulinkonzentrationen feststellen. 
Neben den Parametern des Knochenstoffwechsels spielen auch hormonelle Regelkreise eine Rolle bei der Knochenentwicklung. Eine besondere Bedeutung bei der Proliferation und Differenzierung der Knorpelzellen scheinen das Wachstumshormon (GH) und der Insulin-like growth factor I (IGF-I) zu haben. Das Wachstumshormon ist außerdem für die Kontrolle des Wachstums zuständig, indem es Zellwachstum und- vermehrung fördert. SLOET VAN OLDRUITENBORGHOSTERBAAN et al. (1999) untersuchten den möglichen Zusammenhang zwischen IGF-I, IGF-II, Parathormon, Vitamin D, Cortisol sowie verschiedenen Mengenelementen und dem Auftreten von Osteochondrose. Die Ergebnisse zeigten, dass die OC positive Fohlen eine signifikant niedrigere IGF-I Aktivität gegenüber OC negativen Fohlen aufwiesen. Auch im Bereich des Parathormons, des Vitamin Ds, sowie bei Kupfer, Zink und Calcium zeigten sich signifikante Unterschiede zwischen Osteochondrose negativen und positiven Fohlen. Die Schilddrüsenhormone spielen eine wichtige Rolle bei der Reifung des Knorpels. Bei einer Schilddrüsenunterfunktion können Osteochondrose ähnliche Veränderungen am Skelettsystem beobachtet werden (IRVINE, 1984). Das Parathormon (PTH) fungiert im Blut als wichtigster Regulator des Blut-Calcium-Spiegels. Der antagonistische Gegenspieler des Parathormons ist das Kalzitonin. Ein Anstieg der Kalziumkonzentrationen führt zu einer Hemmung der PTH Freisetzung. Sinkt die Kalzium Konzentration im Blut, mobilisiert das PTH Calcium aus dem Knochen, bis wieder physiologische Blutkonzentrationen erreicht sind.

\subsubsection{Genetische Einflussfaktoren}

PHILIPSSON et al. (1998) sehen die Osteochondrose als eine der häufigsten SkelettErkrankung beim wachsenden Pferd an, zu deren Entstehung auch genetische Faktoren beitragen können. Auch HULTGREN et al. (1988), zählen die Osteochondrose $\mathrm{zu}$ den Erkrankungen mit multifaktorieller Ätiologie unter Integration einer möglichen genetischen Beteiligung. Diese Annahme einer möglichen Beteiligung genetischer Faktoren an der Entstehung der Osteochondrose führte zu zahlreichen populationsgenetischen Studien mit dem Ziel, den genetischen Hintergrund der Osteochondrose zu erforschen. 
Die verschiedenen Heritabilitätsschätzungen lieferten zum Teil stark voneinander abweichende Ergebnisse, die in der folgenden Tabelle 3 dargestellt sind.

Tabelle 3: Überblick über bisherige populationsgenetische Studien zur Osteochondrose

\begin{tabular}{|l|l|l|}
\hline \multicolumn{1}{|c|}{ Autor } & Population und Anzahl Tiere & Heritabilität \\
\hline $\begin{array}{l}\text { SCHOUGAARD et al. } \\
(1987)\end{array}$ & $\begin{array}{l}\text { Dänische Traber } \\
(\mathrm{n}=325)\end{array}$ & 0,26 \\
\hline $\begin{array}{l}\text { GRONDAHL und DOLVIK } \\
(1990)\end{array}$ & $\begin{array}{l}\text { Norwegische Traber } \\
(\mathrm{n}=644)\end{array}$ & 0,32 \\
\hline $\begin{array}{l}\text { PHILIPSSON et al. } \\
(1993)\end{array}$ & $\begin{array}{l}\text { Schwedische Traber } \\
(\mathrm{n}=793)\end{array}$ & $0,09-0,24$ \\
\hline $\begin{array}{l}\text { KWPN } \\
(1994)\end{array}$ & $\begin{array}{l}\text { Holländisches Warmblut } \\
(\mathrm{n}=590)\end{array}$ & $0,01-0,14$ \\
\hline $\begin{array}{l}\text { WINTER } \\
(1995)\end{array}$ & $\begin{array}{l}\text { Deutsche Reitpferderassen } \\
(\mathrm{n}=3566)\end{array}$ & $0,06-0,07$ \\
\hline $\begin{array}{l}\text { WILLMS } \\
(1998)\end{array}$ & $\begin{array}{l}\text { Holsteiner Stuten } \\
(\mathrm{n}=472)\end{array}$ & 0,34 \\
\cline { 2 - 4 } & $\begin{array}{l}\text { Holsteiner Fohlen } \\
(\mathrm{n}=144)\end{array}$ & $0,00-0,21$ \\
\hline $\begin{array}{l}\text { RICARD et al. } \\
(2002)\end{array}$ & $\begin{array}{l}\text { Französisches Warmblut } \\
(\mathrm{n}=733)\end{array}$ & $0,09-0,14$ \\
\hline $\begin{array}{l}\text { PIERAMATI et al. } \\
(2003)\end{array}$ & $\begin{array}{l}\text { Italienisches Warmblut } \\
(\mathrm{n}=350)\end{array}$ & $0,005-0,235$ \\
\hline $\begin{array}{l}\text { SCHOBER } \\
(2003)\end{array}$ & Deutsche Reitpferderassen \\
\hline $\begin{array}{l}\text { STOCK } \\
(2004)\end{array}$ & Hannoversche Warmblutpferde & $0,12-0,33$ \\
\hline
\end{tabular}

HOPPE und PHILIPPSSON (1985) vermuteten anhand ihrer an einer Gruppe Traber und Warmblüter durchgeführten Studie eine erbliche Disposition für Osteochondrose, da signifikante Unterschiede zwischen verschiedenen Hengstnachkommenschaften bestanden. Die gewonnenen Ergebnisse sind jedoch aufgrund geringer Nachkommenzahl pro Vater statistisch nicht abgesichert. SCHOUGAARD et al. (1987) schätzten in einer Untersuchung an 325 dänischen Traberjährlingen, die von neun Vätern abstammten, Heritabilitäten für OC von 0,26. Durch den hohen Standardfehler der Heritabilität von 0,14 kann dieses Ergebnis aber nur bedingt als aussagefähig angesehen werden. 
GRONDAHL und DOLVIK (1990) führten eine genetische Analyse an 644 norwegischen Traberjährlingen durch, und schätzten mittels einer Halbgeschwisteranalyse Heritabilitäten von $0,13 \pm 0,08$ für das Fesselgelenk und $0,12 \pm 0,32$ für das Sprunggelenk bei einem Standardfehler von 0,12. SANDGREN et al. (1993) konnten in ihrer Studie an 793 anderthalbjährigen Trabern Zusammenhänge zwischen röntgenologischen Veränderungen im Sinne einer Osteochondrose am Sprunggelenk, dem Geburtsmonat sowie der Größe der Pferde nachweisen. Darauf aufbauend schätzten PHILIPPSON et al. (1993) am gleichen Datenmaterial Heritabilitäten für osteochondrotische Veränderungen am Sprunggelenk und für plantare/palmare Fragmente im Fesselgelenk unter Anwendung zweier verschiedener Schätzmethoden. Bei Verwendung eines Schwellenwertmodells lagen die Schätzwerte für beide Merkmale in einem Bereich von 0,08-0,09 $\pm 0,07$. Unter Annahme einer normalverteilten Merkmalsausprägung kam man für das Sprunggelenk auf Schätzwerte im Bereich von 0,24 $\pm 0,19$ und für das Fesselgelenk auf Werte von 0,19 \pm 0,13. Aufgrund dieser Ergebnisse kamen PHILIPSSON et al. (1993) zu dem Schluss, dass Befunde für die unterschiedlichen Lokalisationen möglicherweise unabhängig voneinander vererbt werden. Der niederländische Warmblutzuchtverband (KWPN) schätzte 1994 an drei- und vierjährigen Nachkommen von 30 niederländischen Warmbluthengsten Schätzwerte der Heritabilitäten im Bereich von 0,01 unter Anwendung des Halbgeschwistermodells und von 0,04 bei Verwendung des Tiermodells. WINTER (1995) kam anhand seiner Untersuchungen an 3566 deutschen Auktionspferden zu Heritabilitäten für OC im Sprunggelenk im Bereich 0,06 $\pm 0,04$ bis $0,07 \pm 0,03$ bei Anwendung verschiedener Modelle. Aus der Tatsache, dass die Schätzwerte im niedrigen Bereich liegen, schloss WINTER (1995), dass die Erkrankung vor allem durch Umwelteinflüsse verursacht wird. WILLMS (1998) analysierte in seinen Untersuchungen röntgenologisch erhobene Gliedmaßenbefunde von 427 dreijährigen Holsteiner Stuten und 144 zufällig ausgewählten Holsteiner Fohlen, die im Alter von sechs Monaten, einem Jahr sowie zweijährig erneut untersucht wurden. In der Studie wurden sowohl ein Vater- als auch ein Tiermodell verwendet. Bei Anwendung des Vatermodells lagen die Schätzwerte bei den Stuten im Bereich von 0,45 $\pm 0,23$ und bei 0,34 $\pm 0,06$ für das Tiermodell. 
Die Ergebnisse der Fohlen lagen im Vergleich zu den Stuten etwas niedriger bei 0,58 \pm 0,15 unter Anwendung des Vatermodells und bei 0,19 $\pm 0,02$ für das Tiermodell. In einer Studie von RICARD et al. (2002) wurden die Ursachen von Knochen- und Gelenkserkrankungen im Hinblick auf ihre Erblichkeit und ihre Beziehung zu Leistungskriterien an 733 Pferden abstammend von 103 Vätern der Rasse Selle Français untersucht. Es konnten Heritabilitäten im Bereich von 0,0 bis 0,21 für unterschiedliche Lokalisationen in verschiedenen Gelenken geschätzt werden. PIERAMATI et al. (2003) kamen anhand ihrer Untersuchung an 350 Nachkommen von 75 unterschiedlichen Vätern der italienischen Warmblutpopulation (Maremmano) zu Schätzwerten im Bereich von 0,14 $\pm 0,22$ bei Verwendung eines gemischten Tiermodells und im Schwellenwertmodell zu Werten im Bereich von $0,09 \pm 0,24$. SCHOBER (2003) analysierte im Rahmen einer aktuellen großangelegten interdisziplinären Studie zur Osteochondrose röntgenologische Befunde von 629 Fohlen im Mindestalter von fünf Monaten sowie von 591 Müttern dieser Fohlen. 64,5 \% der Fohlen konnten im Alter von zwei Jahren für eine Folgeuntersuchung gewonnen werden. SCHOBER (2003) schätzte anhand dieses Datenmaterials unter Verwendung zweier Modelle Heritabilitäten zwischen 0,16 \pm 0,10 und 0,17 $\pm 0,08$ für das Auftreten von OCD im Fesselgelenk beim Fohlen. Aufgrund nicht erfassbarer potentieller Einflussfaktoren lieferten die Zweijährigen $(0,06 \pm 0,10)$ und die Stuten $(0,02 \pm 0,07)$ vergleichsweise niedrigere Schätzwerte für die gleiche Lokalisation im Fesselgelenk. Für das Auftreten von Osteochondrose am Sprunggelenk wurden unter Zugrundelegung eines Tiermodells Schätzwerte zwischen 0,08 $\pm 0,08$ und 0,09 $\pm 0,05$ für die Fohlen ermittelt. Die Ergebnisse bestätigten sich weitgehend in den Schätzwerten der Zweijährigen $(0,14 \pm 0,07)$ und Stuten $(0,07 \pm 0,08)$. Bei der Untersuchung der OC im Kniegelenk ergaben sich Heritabilitätsschätzwerte bei Zweijährigen und Stuten im Wert von nahezu Null. Diese Ergebnisse sind möglicherweise auf die sehr niedrige Frequenz der Osteochondrose in diesem Gelenk zurückzuführen. Zwischen dem OC-Status im Fohlenalter und im Alter von zwei Jahren ergaben sich für OC Fesselgelenk $\left(r_{g}=0,84\right)$ und im Sprunggelenk $\left(r_{g}=0,98\right)$ Korrelationen in einem sehr hohen Bereich. 
STOCK (2004) analysierte die Ergebnisse einer standardisierten röntgenologischen Gliedmaßenuntersuchung an 3.725 Hannoverschen Warmblutpferden, die in den Jahren 1991-1998 für Reitpferdeauktionen des Hannoverschen Verbandes ausgewählt worden waren. In die Untersuchung einbezogene röntgenologische Veränderungen waren isolierte röntgenologische Verschattungen in Fessel- und Sprunggelenk, deformierende Arthropatien der Sprunggelenke und röntgenologische Strahlbeinveränderungen. Für diese Röntgenbefunde wurde durchgängig mittlere Heritabilitäten in der Größenordnung von $\mathrm{h}^{2}=0,12-0,33$ geschätzt.

Die Ergebnisse der Studien ergeben unter Vorraussetzung verschiedener Definitionen der Erkrankung eine heterogene Datengrundlage, so dass eine eindeutige Zuordnung der Osteochondrose zu den Erbkrankheiten nicht möglich ist (NICHOLAS, 2000), sondern eher die Annahme einer multifaktoriellen Pathogenese angemessen erscheint (JEFFCOTT, 1991). Die Ergebnisse zeigen aber auch eine genetische Beteiligung an der Entstehung der Osteochondrose in einem mittleren Bereich von $\mathrm{h}^{2}=0,1-0,3$, ein Wert, der von verschiedenen Autoren als ausreichend erachtet wird, um eine erfolgreiche züchterische Bearbeitung der Osteochondrose $\mathrm{zu}$ gewährleisten (BRUNS, 2001).

\subsection{Auswirkungen der OC}

Um die Implementierung der Selektion gegen Osteochondrose in Zuchtprogramme im Hinblick auf die Nutzungsdauer von Reitpferden und in diesem Zusammenhang auf die ökonomischen Auswirkungen richtig bewerten $\mathrm{zu}$ können, sollten die Einflüsse der Osteochondrose auf diese Bereiche berücksichtigt werden. Besonders wichtig für die Selektionsarbeit und damit einhergehenden züchterischen Überlegungen in einem modernen Reitpferdezuchtprogramm sind Beziehungen zwischen funktionalen und Leistungs- sowie Exterieurmerkmalen.

\subsubsection{Beziehungen zwischen orthopädischen Defekten und}

\section{Leistungs- sowie Exterieurmerkmalen}

Der Zuchtausschluss von Pferden, die einen OC-Befall aufweisen, könnte dazu führen, dass wichtige und nutzvolle Eigenschaften dieser Pferde in Bezug auf Leistungsmerkmale wie Dressur und Springen für die Pferdezucht verloren gingen. 
Deshalb ist es in diesem Zusammenhang besonders wichtig festzustellen, ob Korrelationen zwischen orthopädischen Defekten und Leistungs- sowie Exterieurmerkmalen bestehen.

\subsubsection{Leistungsmerkmale}

Auf die besondere Bedeutung von Beziehungen zwischen Gliedmaßenveränderungen und Leistungsmerkmalen im Hinblick auf züchterische Fragestellungen weisen WINTER (1995) und WILLMS (1999) in ihren Ausführungen hin. Beide Autoren beschäftigten sich mit genetischen und phänotypischen Korrelationen zwischen Osteochondrose und Kriterien der Hengst (WINTER)- und Stutenleistungsprüfung (WILLMS). Die Ergebnisse dieser Studien werden in der folgenden Tabelle 4 dargestellt.

Tabelle 4: Überblick über die Ergebnisse der Studien von WILLMS (1995) und WINTER (1998)

\begin{tabular}{|c|c|c|c|}
\hline \multicolumn{2}{|c|}{} & WINTER (1995) & WILLMS (1998) \\
\hline \multirow{2}{*}{ Rittigkeit } & rg & 0,41 & 0,14 \\
\cline { 2 - 4 } & rp & 0,00 & - \\
\hline \multirow{2}{*}{ Springen } & rg & 0,53 & 0,13 \\
\cline { 2 - 4 } & rp & $-0,04$ & - \\
\hline
\end{tabular}

WINTER (1995) griff aufgrund nicht vorhandener Leistungsinformationen der in dieser Studie untersuchten Auktionspferde, auf Verwandtenleistung (Väter) zurück. Im Rahmen seiner Untersuchung an Merkmalen der Hengstleistungsprüfung stellte er aufgrund der festgestellten positiven genetischen Korrelationen zwischen den Gliedmaßenerkrankungen und den Leistungsmerkmalen eine ungünstige Beziehung fest. Die Verbesserung der Noten in der Rittigkeit und im Springen führe zu einer Verschlechterung der Gliedmaßenerkrankungen. WILLMS (1998) untersuchte genetische Korrelationen zwischen Merkmalen der Stutenleistungsprüfung und Gliedmaßenveränderungen. Er berechnete leicht positive genetische Beziehungen $(0,13-0,14)$ mit relativ hohen Standardfehlern, die durch geringe Tierzahlen zustande kamen. 
BREHM und STAECKER (1994, 2000) analysierten den Einfluss osteochondrotischer Veränderungen im Sprunggelenk auf die Rennleistung von zwei-, drei-, und vierjährigen Trabrennpferden. Der Vergleich der Rennleistung OC positiver und nicht befallener Pferde brachte weder hinsichtlich der Starts, der Anzahl der Platzierungen noch des Kilometerrekords signifikante Unterschiede hervor. SCHOBER (2003) stellte im Rahmen ihrer Untersuchungen an Exterieurund Leistungszuchtwerten aller Prüfungsformen von Hengsten und Stuten aus dem Jahre 2002 keine Hinweise auf eine Beeinträchtigung der Gesundheit bezüglich OC bei verstärkter Berücksichtigung der verschiedenen Leistungsparameter (Dressur und Springen) fest.

\subsubsection{Exterieurmerkmale}

Ein korrekter Körperbau wird im Allgemeinen als Vorraussetzung für eine optimale Leistungsentwicklung angesehen (NISSEN, 1986). Aus diesem Grund konnte sich eine systematische Exterieurbeurteilung im Laufe der Zeit in den Zuchtprogrammen der verschiedenen Pferdezuchtverbände fest etablieren und wird bei Körungen, Stuteneintragungen sowie bei Stuten- und Fohlenschauen durchgeführt (WILLMS, 1998). Dabei werden die verschiedenen Merkmalskomplexe des Exterieurs mit Hilfe eines Notenschemas bewertet. Beziehungen zwischen den Gliedmaßenerkrankungen und dem Exterieur sind für das frühzeitige Erkennen von Gliedmaßendefekten besonders wichtig. Die frühzeitige Datengewinnung der Exterierbewertung auf Fohlenschauen und Stutbucheintragungen könnte das teuere und aufwendige Röntgenverfahren ergänzen bzw. ersetzen (WINTER, 1995). WINTER (1995) stellte in seinen Untersuchungen eine negative genetische Korrelation $r_{g}$ von $-0,12$ für die Osteochondrose im Sprunggelenk und den Hintergliedmaßen sowie eine phänotypische Korrelation $r_{p}$ von 0,06 fest. WILLMS (1998) berechnete eine leicht negative Beziehung $(-0,15)$ zwischen den Merkmalen Breite und Tiefe des Körpers zur Osteochondrose. SCHOBER (2003) konnte keine relevanten Beziehungen zwischen der Qualität des Exterieurs und dem Auftreten von Osteochondrose feststellen und schließt daraus, dass das bestehende Beurteilungsschema die Erkrankung nicht negativ zu beeinflussen scheint. 


\subsubsection{Einfluss der OC auf die Nutzungsdauer von Sportpferden}

Die Behandlung OC- befallener Pferde mit klinischen Symptomen kann konservativ mit Hilfe entzündungshemmender Präparate, oder chirurgisch erfolgen, hier werden mittels einer Arthroskopie die freien Gelenkkörper entfernt. HERTSCH (2002) sieht die operative Behandlung als die sicherste Methode für einen langfristigen und dauerhaften Heilungserfolg an.

Ein besonders wichtiger und interessanter Aspekt ist die Nutzungsdauer OCbefallener Pferde. In diesem Zusammenhang durchgeführte Untersuchungen wurden bisher meist an Rennpferden durchgeführt, da deren Leistung einfacher zu objektivieren ist (Rennzeiten, Anzahl Starts und/oder Siege, Gewinnsummen) als die eines Reitpferdes (STOCK, 2004). LAWS et al. (1993) verglichen Pferde mit Osteochondrose Befund im Sprunggelenk mit OC- freien Pferden, die sich im Alter und Geschlecht entsprachen, hinsichtlich der Anzahl der Starts und der Gewinnsumme. Die Osteochondrose befallenen Tiere hatten signifikant weniger Starts als die gesunden Tiere und damit auch geringere Gewinnsummen. BEARD et al. (1994) untersuchten an 109 Voll- und Warmblütern mit osteochondrotischen Veränderungen im Sprunggelenk deren postoperative Rennleistung. Sowohl die Anzahl der Starts als auch die Höhe der Gewinnsumme der chirurgisch behandelten Pferde war geringer. Außerdem zeigte sich, dass Pferde mit multiplen Defekten weniger häufig an Rennen teilnahmen als Pferde mit nur einem Befund. GRONDAHL und ENGELAND (1995) analysierten den Einfluss radiologischer Veränderungen auf die Rennleistung von 753 Trabern. Die Ergebnisse zeigten, dass Pferde mit röntgenologisch sichtbaren Veränderungen weit weniger Rennen bestritten und dementsprechend auch weniger Gewinne erzielten. STOCK (2004) untersuchte genetische Beziehungen zwischen röntgenologischen Gliedmaßenbefunden und dem Einsatz der Pferde im Reitsport. Als Leistungsparameter wurden die Anzahl Nennungen pro Jahr und die Anzahl Platzierungen pro Jahr definiert, anhand derer Aussagen über die Kontinuität der Einsetzbarkeit und über die erbrachte Leistung getroffen werden konnten. Als röntgenologische Veränderungen wurden isolierte Verschattungen im Fessel- und Sprunggelenk, deformierende Arthropathien der Sprunggelenke und röntgenologische Strahlbeinveränderungen in die Untersuchung einbezogen. 
Die festgestellten additiv-genetischen Korrelationen deuten auf einen negativen Zusammenhang zwischen dem Auftreten röntgenologischer Veränderungen im Gliedmaßenbereich und der Leistung von Reitpferden hin.

\subsubsection{Wirtschaftliche Bedeutung der OC}

Mit der Einführung des neuen Pferdekaufrechts zum 1.1.2002 hat der gesundheitliche Status des Pferdes beim Verkauf eine noch größere Bedeutung erlangt, da der Verkäufer nun die Verpflichtung hat, dem Käufer eine mangelfreie „Sache“ zu verschaffen. Die Gliedmaßenerkrankung Osteochondrose und ihre besondere Form die Osteochondrosis dissecans stellten in den vergangenen Jahren eine häufige Diagnose bei Ankaufsuntersuchungen dar, da mit Hilfe der bildgebenden Diagnostik eine detaillierte Aussage über den röntgenologischen Status des Pferdes möglich ist. Die gewinnbringende Vermarktung des Pferdes wird durch das Auftreten röntgenologischer Abweichungen deutlich beeinträchtigt.

DOHMS (2003) kalkuliert die Produktionskosten für ein dreijähriges angerittenes Reitpferd mit ca. $6250 €$. Bei einem mit Osteochondrose befallenen Pferd fallen zusätzliche Kosten für Lahmheitsuntersuchung, Röntgenbilder, Operation und Nachsorge an, die im Folgenden genauer aufgeschlüsselt sind. Die Kosten wurden anhand der Gebührenordnung für Tierärzte (GTO) unter Annahme des einfachen Satzes berechnet.

\section{Lahmheitsuntersuchung:}

Große Röntgenuntersuchung:

(18 Aufnahmen)

\section{Gelenkorthopädische Operation:}

je nach Gelenk und Aufwand der Operation inkl. Narkose,

künstliche Beatmung, angewendete Arzneimittel,

Verbrauchsmaterialien

Nachsorge:

$266,9 €$

inkl. angewendete Arzneimittel, Verbrauchsmaterialien,

Stallkosten (berechnet für 10 Tage)

$16 \%$ Mehrwertsteuer

Gesamtkosten
$25,56 €$

$296,56 €$

(GTO)

(GTO)

$800 €$

(GTO) 
Verzögerungen bei der Ausbildung und daraus folgende Gewinnverluste bei Turnierstarts sowie Beeinträchtigungen durch mögliche Spätfolgen können hier nicht kalkuliert werden. Beim Verkauf des Pferdes hat der Züchter möglicherweise zusätzlich mit deutlichen Mindereinnahmen aufgrund des positiven OC Befundes des Pferdes zu rechnen, da der OC Befall zu einer Einteilung in die Röntgenklasse III-IV führen kann (vergleiche dazu Kapitel: Diagnose der Osteochondrose anhand von Röntgenbildern). In der Praxis zeigt sich, dass Pferde, die aufgrund ihres orthopädischen Status in die Röntgenklasse III-IV eingeteilt wurden, kaum noch oder nur sehr schwer zu verkaufen sind (GENN, 2005). BATHE (1994) sieht in Erkrankungen des Pferdes, die aufgrund von Verletzungen oder Entwicklungsstörungen entstehen, ein großes ökonomisches Problem, die in erhöhten Abgangsraten und wirtschaftlichen Verlusten resultieren. VAN HOEGMOOD (2003) weist darauf hin, dass die Verkaufsfähigkeit und der Verkaufswert eines Pferdes von seinem Röntgenstatus abhängig sind. Auch CHRISTMANN (2005) spricht röntgenologischen Befunden eine große wirtschaftliche Bedeutung $\mathrm{zu}$. GLAWATZ (2004) stellte im Rahmen ihrer Untersuchungen über den Einfluss des OC-Status auf die Preisgestaltung des hannoverschen Hengstmarktes eine nicht unwesentliche Beeinflussung des Marktes durch die Osteochondrose fest. Zudem wurde ein deutlich negativer Effekt von OC auf die Körgesamtnote festgestellt.

Somit zeigt sich, dass aufgrund der schwierigen Absatzlage auf dem Pferdemarkt eine besondere Selektion auf Gesundheitsmerkmale immer mehr an Bedeutung gewinnt und in die Selektionsentscheidungen des praktischen Pferdezüchters verstärkt mit einbezogen werden sollten.

\subsection{Zuchtplanung in der deutschen Reitpferdezucht}

Da das letztendliche Ziel dieser Arbeit sein soll, die Selektion gegen Osteochondrose in Zuchtprogramme $\mathrm{zu}$ implementieren, werden im Folgenden die Selektionsmethoden und Zuchtmaßnahmen in der deutschen Reitpferdezucht erläutert, um Ansatzpunkte für die Integration einer züchterischen Bearbeitung der Erkrankung aufzuzeigen. 
Das einheitliche Rahmenzuchtziel des deutschen Reitpferdes wird von allen deutschen Zuchtverbänden folgendermaßen formuliert: „Gezüchtet wird ein edles, großliniges und korrektes, gesundes und fruchtbares Reitpferd mit schwungvollen, raumgreifenden und elastischen Bewegungen, das aufgrund seines Temperamentes, seines Charakters und seiner Rittigkeit für Reitzwecke jeder Art geeignet ist“ (Zuchtverbandsordnung, ZVO, 2005). Insgesamt sind in Deutschland 72693 Warmblut Zuchtstuten und 3884 Warmblut Zuchthengste registriert, die von 16 Warmblutzuchtverbänden betreut werden (JAHRESBERICHT FN, 2004). Die Selektion der Stuten und Hengste erfolgt durch verschiedene Leistungsüberprüfungen, die im Folgenden beschrieben werden.

\subsubsection{Hengstselektion}

HARING (1980) stellte einen allgemeinen Zuchtplan für die deutsche Reitpferdezucht auf, dessen einzelne Selektionsstufen sich auf die Auswahl der Hengste beziehen:

1) Die erste Begutachtung und Beurteilung erfolgt im Fohlenalter bei Fuß der Mutter nach den Kriterien Abstammung und Merkmalen der äußeren Erscheinung einschließlich der Grundgangarten.

2) Nach den gleichen Merkmalen erfolgt die Beurteilung im Alter von 2 1/2 Jahren zur Körung.

3) An die Körung schließt sich die Eigenleistungsprüfung auf Station an. Diese Eigenleistungsprüfungen sind Leistungsprüfungen im Sinne des Tierzuchtgesetzes und können als Stationsprüfung, als Turniersportprüfung oder als Kombination aus Veranlagungsprüfung und Turniersportprüfung durchgeführt werden (ZVO, 2004).

4) Nachkommenprüfung im Turniersport. Gesetzlich vorgeschrieben sind die Selektionsstufen zwei und drei (HARING, 1980).

Da das in dieser Arbeit entwickelte Simulationsprogramm vor allem die Selektion der Hengste bis zur Durchführung der Körung im Alter von 2 1/2 Jahren simulieren soll, wird dieses Verfahren am Beispiel des hannoverschen Zuchtgebietes näher beschrieben. Pro Jahr werden im hannoverschen Zuchtgebiet ungefähr 5000 Hengstfohlen geboren. Aufgrund der Auswahl der Züchter hinsichtlich der Qualität der Junghengste werden ca. 750 Hengste pro Jahr zu den Auswahlterminen, 
die der Körung vorgeschaltet sind, vorgestellt. Bei diesen Auswahlterminen wird die Qualität der Hengste im Freispringen, ihre Grundgangarten und die Korrektheit ihres Exterieurs überprüft. Die Hengste müssen mindestens zwei Jahre alt sein und über eine vom Hannoveraner Verband anerkannte Abstammung verfügen. Zusätzlich müssen eine tierärztliche Untersuchung und eine Abstammungsüberprüfung der Hengste erfolgen. Aus den ca. 750 vorgestellten Hengsten werden ca. 100 Hengste aufgrund ihrer Qualität ausgewählt, die zur Hauptkörung in Verden vorgestellt werden. Die vorausgewählten 100 Hengste werden bei der Körung an zwei Tagen im Freispringen, auf der Dreiecksbahn und auf dem Pflaster bewertet. Für ein positives Körurteil muss mindestens eine Gesamtnote von 7,0 erreicht werden (VERBAND HANNOVERSCHER WARMBLUTZÜCHTER, 2005).

\subsubsection{Stutenselektion}

Auch bei den Stuten erfolgt die erste Bewertung im Fohlenalter bei Fuß der Mutter nach den Kriterien Abstammung und Merkmalen der äußeren Erscheinung einschließlich der Grundgangarten. Ab einem Alter von drei Jahren erfolgt die Stutbuchaufnahme, hier werden die Bewertungspunkte:

- Rasse und Geschlechtstyp

- Qualität des Körperbaus

- Korrektheit des Ganges

- Elastizität und Schwung (Trab)

- Schritt

- Gesamteindruck und Entwicklung

mit Noten von 1-10 bewertet, woraus sich dann die Gesamtbewertung errechnet.

Die Zuchtstutenprüfung wird für dreijährige und ältere Stuten durchgeführt. Hier werden die Grundgangarten, die Springanlage beim Freispringen sowie die Rittigkeit durch einen Fremdreiter im Rahmen einer Stations- oder Feldprüfung mit Noten von 1-10 bewertet. Seit 1992 besteht für hannoversche Stuten, die nach dem 30.10.1989 geboren sind, die Pflicht zur Teilnahme an der Zuchtstutenprüfung, um als Hengstmutter anerkannt zu werden (SCHADE, 1992). 
Abbildung 2 verdeutlicht die beschriebenen Selektionsabläufe auf der Stuten- und auf der Hengstseite am Beispiel des Zuchtprogramms des Hannoveraner Verbandes.

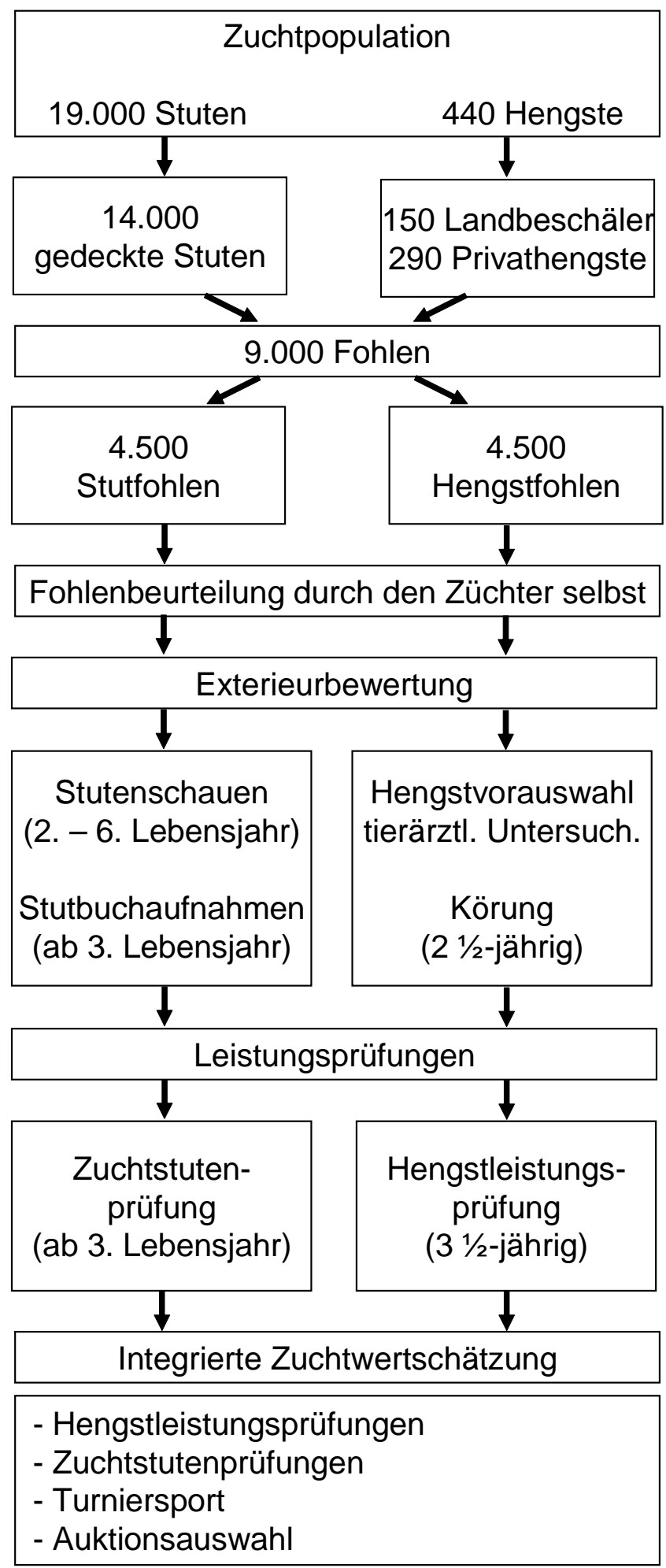

Abbildung 2: Aktuelles Hannoversches Zuchtprogramm

(Quelle: Der Hannoveraner, ein Leitfaden für den Züchter ) 
Die Selektion der Hengste und Stuten mündet in die Zuchtwertschätzung, um die bei den verschiedenen Leistungsprüfungen gewonnenen Informationen im Sinne des Zuchtfortschritts zu nutzen.

\subsubsection{Zuchtwertschätzung in der Reitpferdezucht}

Bei Selektionsentscheidungen im Rahmen der Zuchtplanung ist es von entscheidender Bedeutung, zum frühestmöglichen Zeitpunkt Informationen über die zu erwartenden Zuchtwerte potentieller Elterntiere zur Verfügung zu haben. Diese Informationen werden über die Zuchtwertschätzung gewonnen. Der Zuchtwert eines Pferdes ist seine genetisch begründete Leistungsabweichung gegenüber Vergleichstieren aus der Population. Um die Zuchtwerte von potentiellen Elterntieren in der Reitpferdezucht $\mathrm{zu}$ ermitteln, benötigt man Zuchtwertschätzverfahren, die es ermöglichen, die vererbbaren Leistungsunterschiede zwischen Pferden von den umweltbedingten Leistungsunterschieden $\mathrm{zu}$ trennen und $\mathrm{zu}$ quantifizieren. Da der Einfluss eines Hengstes auf die Pferdezucht im Hinblick auf seine Nachkommenzahl höher eingeschätzt wird als der Einfluss der Stutenseite, werden die Methoden der Zuchtwertschätzung vor allem genutzt, um Informationen über Vatertiere zu erhalten (VELSEN-ZERWECK, 1998).

GLODEK und BADE (1975) entwickelten die ersten Selektionskriterien und Zuchtwertschätzmethoden für eigenleistungsgeprüfte Hengste nach populationsgenetischen Gesichtspunkten auf der Basis der Untersuchungen von BADE (1974). Die meisten Verfahren der Varianzkomponenten- und Zuchtwertschätzung wurden für die Milchrinderzucht entwickelt und erst später auf andere Tierarten übertragen. Vorläufer des heute allgemein verwendeten BLUPVerfahrens ist der CC (Contemporary Comparison oder Zeitgefährtenvergleich), der ab 1976 in Frankreich als Zuchtwertschätzverfahren zur Anwendung kam. Der CCTest wurde 1986 durch die von HENDERSON (1973) entwickelte BLUP-Methode (Best Linear Unbiased Prediction) abgelöst. Die Voraussetzung für die Nutzung des BLUP-Verfahrens in der deutschen Pferdezucht wurde durch die Arbeiten von BRUNS (1987) und MEINARDUS (1988) geschaffen. 
MEINARDUS (1988) entwickelte ein Verfahren für die Zuchtwertschätzung von Hengsten anhand der Leistung ihrer Nachkommen im Turniersport. Darauf aufbauend entwickelte VELSEN-ZERWECK (1998) die „Integrierte Zuchtwertschätzung“ für deutsche Reitpferde, bei der Informationen aus verschiedenen Leistungsprüfungen zusammengeführt und für eine Zuchtwertschätzung nutzbar gemacht wurden. Weitere Untersuchungen auf dem Gebiet der Zuchtwertschätzung wurden von WEYMANN (1992) für das Exterieur und von UPHAUS (1993) für die Stutenleistungsprüfung durchgeführt.

Die BLUP-Methode ist nicht von der Annahme eines durchschnittlichen genetischen Niveaus der Vergleichstiere innerhalb einer Vergleichsgruppe abhängig und ermöglicht deshalb die gleichzeitige Schätzung der fixen Effekte und der Zuchtwerte, somit können ungleiche Verteilungen berücksichtigt werden. Durch diese Methode wird deshalb im Vergleich zu anderen Modellen die höchste Korrelation zwischen tatsächlichem und geschätztem Wert ermittelt (KRÄUSSLICH, 1994). KATONA et al. (1983) und ARNASON (1983) sehen die Anwendung der BLUP-Methode für Zuchtwertschätzungsverfahren in der Reitpferdezucht aufgrund der ungünstigen Zuchtstrukturen als sehr geeignet an. Vier BLUP-Varianten gelangten in der Tierzucht zum Einsatz (je nach Art des zufälligen Effektes): 1. das Vatermodell, 2. das Vater-Großvater Modell, 3. das Vater-Mutter Modell und 4. das Tiermodell. Für ARNASON (1983) bietet die Verwendung eines BLUP-Vatermodells bei Vorliegen der Leistungsinformation von mindestens 20 bis 40 Nachkommen pro Vater eine ausreichende Genauigkeit. Aufgrund der teilweise geringen Reproduktionsrate der Hengste zeigte sich jedoch, dass in der Verwendung eines Tiermodells Vorteile liegen. BRUNS (1987) konnte anhand seiner Untersuchungen die Vorteile des Tiermodells zur Ausschaltung der Verzerrungen in Folge assortativer Paarungen belegen. Die Vorteile des Tiermodells benennt WEYMANN (1992) folgendermaßen:

- Genauere Zuchtwerte als beim Vatermodell

- Für Tiere ohne Eigenleistung sind Zuchtwerte über verwandtschaftliche Beziehungen schätzbar

- Korrektur des Anpaarungsniveaus

- Ermittlung des genetischen Trends

- Zuchtwerte aller Tiere direkt vergleichbar 
Mit der Verwendung eines Mehrmerkmals-Tiermodells ist eine weitere Steigerung der Genauigkeit der Zuchtwertschätzung möglich. Bei dieser Modellvariante werden unter Berücksichtigung der Beziehungen zwischen den Merkmalen Zuchtwerte für mehrere Merkmale gleichzeitig geschätzt. Durch die Berücksichtigung der Kovarianzen im Mehrmerkmalsmodell vergrößert sich die Informationsbasis für einen Zuchtwert, zudem werden die Effekte im Modell genauer geschätzt.

Auf Basis der Forschungsergebnisse von VELSEN-ZERWECK (1998) wurde die Zuchtwertschätzung in der deutschen Reitpferdezucht neu entwickelt. Bisher wurde eine Zuchtwertschätzung für die Ergebnisse der Hengstleistungsprüfung, die Stutenleistungsprüfung und den Turniersport durchgeführt. Neu aufgenommen wurden die Daten aus den Aufbauprüfungen des Turniersports, den sogenannten Dressur- und Springpferdeprüfungen. Die neue integrierte Zuchtwertschätzung beinhaltet die Zusammenführung von Leistungsdaten aus den verschiedenen Prüfungsformen; es wird jetzt nicht mehr eine Zuchtwertschätzung für jede Prüfungsform durchgeführt, sondern nur noch eine Zuchtwertschätzung, die Informationen von allen Prüfungsformen gleichzeitig benutzt. Somit können schon für junge Pferde relativ genaue und durch Selektion unverfälschte Zuchtwerte hinsichtlich ihrer Dressur- und Springveranlagung geschätzt werden.

\subsubsection{Die Implementierung von Gesundheitsmerkmalen in Tierzuchtprogramme}

Die züchterische Verbesserung von Gesundheitsmerkmalen und damit der Langlebigkeit stellt ein wichtiges Ziel in der Tierzucht dar. Bei landwirtschaftlich genutzten Tierarten wurden erste Maßnahmen zur Integration von Gesundheitsmerkmalen in Zuchtprogramme ergriffen.

In der Rinderzucht wurde die Aufnahme von Fruchtbarkeit, Kalbeverlauf, Totgeburtenrate, somatischem Zellgehalt und Nutzungsdauer in einem Gesamtzuchtwert für Bullen verwirklicht. Generell ist jedoch in der Rinderzucht ein deutlicher Trend im Rückgang der Lebens- und Nutzungsdauer zu verzeichnen, der sich auch durch die Einführung der funktionalen Nutzungsdauer in den Gesamtzuchtwert bei Milchrinderbullen nicht aufhalten ließ (DISTL, 2003). 
Auch im Hinblick auf die steigende Milchleistung und auch aus ökonomischer Sicht sollte eine vermehrte Berücksichtigung von Gesundheitsmerkmalen stattfinden, da Langzeitselektionsexperimente in den USA zeigten, dass mit steigender Milchleistung die Krankheitskosten deutlich anstiegen (KELM et al., 2003).

In vielen Schweinezuchtprogrammen werden für Mutterrassen/-linien Fruchtbarkeitsmerkmale in Form von lebend geborenen und aufgezogenen Ferkeln berücksichtigt. Der Anomalienindex zur Verminderung von erblichen Defekten hat sich mittlerweile etabliert und zu einer signifikanten Reduktion angeborener Defekte bei Ferkeln beigetragen.

In der Pferdezucht fand bisher die Selektion gegen die Erkrankung „Kehlkopfpfeifen“ in Zuchtprogrammen Berücksichtigung. Kehlkopfpfeifen wird klinisch und pathologisch als eine durch Stimmbandlähmung und durch ein inspiratorisches Atemgeräusch gekennzeichnete Erkrankung des Larynx charakterisiert (RAUF, 1964). MIESNER (1996) beschäftigte sich mit genetischen und umweltbedingten Einflussfaktoren auf das Kehlkopfpfeifen sowie möglichen züchterischen Maßnahmen und stellte bei betroffenen Pferden deutliche Leistungseinbußen und Wertminderungen sowie einen hohen Erblichkeitsgrad der Erkrankung fest $\left(h^{2}=0,61\right)$. Aufgrund dieser Beeinträchtigungen forderte MIESNER (1996) eine verstärkte Berücksichtigung der Selektion gegen diese Erkrankung in der Pferdezucht, da bisher nur junge, neu in die Zucht einbezogene Hengste und Stuten auf diese Erkrankung untersucht und ggf. von der Zucht ausgeschlossen werden.

Die deutsche Pferdezucht konnte und kann große Erfolge in allen Sparten der Reiterei verbuchen, da in wichtigen Leistungseigenschaften wie Grundgangarten, Rittigkeit, Springanlage und Interieur große Zuchtfortschritte erzielt werden konnten. Um den zunehmenden Anforderungen und Belastungen der Pferde sowie der angespannten Lage auf dem Pferdemarkt auch im Hinblick auf das geänderte Kaufrecht Herr zu werden, sollte eine stärkere Berücksichtigung von funktionalen Kriterien und Gesundheitsmerkmalen bei züchterischen Entscheidungen erfolgen (GLODEK, 1979; WINTER, 1995; WILLMS, 1998; PHILIPSSON, 1999; CHRISTMANN, 2000; BRUNS, 2002; SCHOBER, 2003). 
Nach MARAHRENS (1993) hat ein Merkmal, das in Selektionsentscheidungen einbezogen werden soll, folgende Kriterien zu erfüllen:

1) objektive und genaue Feststellbarkeit

2) erbliche Prädisposition

3) wirtschaftliche Bedeutung

Hinzuzufügen $\mathrm{zu}$ den Kriterien sind noch genetisch-statistische Parameter eines Merkmals wie die Varianz und Korrelationen zu anderen Merkmalen. Wie die vorherigen Ausführungen gezeigt haben, „erfüllt“ die Osteochondrose diese Bedingungen, wurde jedoch bisher nur von einzelnen Zuchtverbänden in deren Zuchtprogramm berücksichtigt. Diese geringe Berücksichtigung von Gesundheitsmerkmalen in Zuchtprogramme sieht PHILIPSSON (1999) darin begründet, dass Leistungsmerkmale relativ leicht $\mathrm{zu}$ definieren sind, während Mängel in der Konstitution verschiedene Ursachen haben können, die es unter Berücksichtigung ihrer Häufigkeit und relativen Bedeutung zu quantifizieren gilt. Die Genauigkeit der Diagnose und des Erbgangs sind weitere wichtige Komponenten für die Entwicklung effektiver Selektionspläne. PHILIPSSON (1999) beschreibt weiterhin, dass in Populationen, in denen die Hauptselektion bei jungen Pferden stattfindet, bevor nennenswerte Leistungen erbracht wurden, die natürliche Selektion gegen die Erkrankung nicht greifen kann. Der einzige Weg zur Verbesserung des Gesundheitsstatus ist die direkte Berücksichtigung des Defekts in der Selektion.

\subsubsection{Die Implementierung der Selektion gegen OC in}

\section{Pferdezuchtprogramme}

Verschiedene Pferdezuchtverbände haben die tierzüchterische und finanzielle Relevanz der Osteochondrose erkannt, und die Selektion gegen diese Erkrankung in ihr Zuchtprogramm integriert.

Das Zuchtziel für Reitpferde im Königlichen Warmblutstutbuch der Niederlande (KWPN) zielt auf ein in allen Disziplinen des Reitsports begabtes Pferd ab, kombiniert mit einem funktionellen und ästhetischen Exterieur, geschmeidigen Bewegungen und einer guten Gesundheit. Als besonders wichtiges Gesundheitsmerkmal wird die Osteochondrose angesehen. 
Deshalb findet seit 1987 eine Selektion der Hengste bezüglich ihres radiologischen Status für OC statt. Pro Jahr werden im niederländischen Zuchtgebiet ca. 5000 Hengstfohlen geboren. Während der Aufzucht werden ungefähr 5\% aufgrund von Osteochondrose und weitere 10\% aus anderen Gründen für die Körung ausselektiert. Bei der Vorauswahl zur Körung werden ca. 600 2,5 jährige Hengste vorgestellt, 80\% der Hengste wurden von ihren Züchtern bereits auf OC getestet. Aus den 600 Hengsten werden 74 Hengste für die Körung ausselektiert, diese Hengste werden auf OC und weitere gesundheitliche Defekte (z.B. Samenqualität) getestet, so dass ungefähr 60 Hengste die zentrale Hengstleistungsprüfung absolvieren, die den letzten Schritt bei der niederländischen Hengstselektion darstellt. Ingesamt gesehen werden so ca. $25 \%$ eines Körjahrgangs auf der letzten Selektionsstufe aufgrund eines OC Befalls von weiteren Selektionsentscheidungen ausgeschlossen (KOENEN et al., 2000). Auch in der italienischen Reitpferdezucht wird eine Überprüfung des OC Status im Rahmen der Hengstleistungsprüfung durchgeführt, OC positive Hengste werden von der Selektion ausgeschlossen. Auch auf der Stutenseite wird während der 30tägigen Stutenleistungsprüfung eine Untersuchung auf Osteochondrose durchgeführt. OC befallene Stuten können keine Hengstmütter werden (PIERAMATI et al., 2003).

Das Szenario der Integration der Selektion gegen Osteochondrose in ein Zuchtprogramm wurde in verschiedenen Studien mittels Simulationsprogrammen simuliert. Um die Effizienz der derzeitig in den Niederlanden angewandten Selektionsmethode $\mathrm{zu}$ überprüfen, verglichen KOENEN et al. (2000) drei unterschiedliche Selektionsstrategien gegen Osteochondrose anhand einer simulierten Population, die aus 15.000 Stuten und 200 Hengsten bestand, die jährlich 10.600 Fohlen produzieren. Die Osteochondrose- Frequenz betrug 25\% in dieser Population. Die erste Selektionsstrategie sah keine Selektion gegen Osteochondrose vor. Bei der zweiten Alternative wurden auch die Stuten in die Selektion gegen Osteochondrose mit einbezogen. In der dritten Strategie fand eine Nachkommenprüfung der Hengste hinsichtlich eines Ostechondrose-Befalls statt. Die Ergebnisse zeigten, dass die Frequenz der Osteochondrose bei 21\% liegt, wenn keine Selektion gegen die Erkrankung durchgeführt wird. Die anderen beiden Selektionsmethoden führten zu einer Verringerung der Osteochondrose-Frequenz auf 17\%. Die Simulationsergebnisse wurden auf einen Zeitraum von 50 Jahren bezogen. 
Auch PIERAMATI et al. (2003) führten eine Simulationsstudie in der italienischen Maremmano Population durch, um verschiedene Selektionsmethoden gegen Osteochondrose hinsichtlich ihrer Effektivität $\mathrm{zu}$ überprüfen. Es wurden eine Ausgangsfrequenz von $16 \%$ und verschiedene Heritabilitäten (9\% und 14\%) für Osteochondrose angenommen. Die simulierten Selektionsszenarien sahen folgendermaßen aus:

1) Selektion von Hengsten und Stuten

2) Selektion OC positiver Hengste bei der Prüfung auf Station

3) Selektion OC positiver Hengste und Hengstmütter

Das erste Szenario, in das die Selektion beider Geschlechter einbezogen wurde, brachte eine Reduktion der Frequenz auf ca. 2\%. Bei den beiden anderen Selektionsmethoden wurde eine Verringerung der Häufigkeit von $16 \%$ auf ca. $6 \%$ in einem Zeitraum von fünf Generationen festgestellt.

Als Konsequenz aus dem OC-Forschungsprojekt verschiedener deutscher Universitäten hat der Verband der Züchter des Hannoveraner Pferdes im Jahre 2004 als erster Verband im deutschen Zuchtgebiet den Osteochondrose-Status eines Hengstes als Kriterium für die Auswahl zur Körung erklärt. Hengste mit großen osteochondralen Fragmenten im Sprung- und Fesselgelenk werden nicht zur Körung zugelassen. Das Kniegelenk muss zudem völlig befundfrei sein. Mit Ausnahme des Kniegelenks wird nicht die absolute Befundfreiheit verlangt, sondern die Befunde sollen keine Beeinträchtigung der Nutzungsfähigkeit erwarten lassen. Operationen zur Entfernung von osteochondralen Fragmenten müssen vom Besitzer des Hengstes angegeben werden. Nicht jeder Hengst, der einen Befund aufweist, wird von vorneherein ausselektiert, da vermieden werden soll, dass durch eine $\mathrm{zu}$ strenge tierärztliche Vorauswahl der Bewegungsspielraum hinsichtlich der Selektion auf Dressur und Springen zu sehr eingeschränkt wird (CHRISTMANN, 2004). Die Auswirkungen dieser Selektionsmaßnahmen hinsichtlich der Reduktion der OCFrequenz innerhalb müssen abgewartet werden. Da die Population im Rahmen dieser Simulationsstudie in Anlehnung an Strukturverhältnisse aus der Pferdezuchtpraxis erstellt wurde, können die hier erzielten Ergebnisse einen Ausblick auf möglicherweise in der Zukunft erzielte Selektionserfolge innerhalb der Hannoveraner Population geben. 


\section{Material und Methoden}

\subsection{Zielsetzung}

Die vorliegende Arbeit hat das Ziel, anhand eines Computersimulationsprogramms die Auswirkungen von Selektionsstrategien gegen die Gelenkserkrankung Osteochondrose auf eine simulierte Pferdepopulation nachzuvollziehen. $\mathrm{Zu}$ diesem Zweck wurde eine Population erstellt, die sich an realen Zuständen aus der Pferdezuchtpraxis orientiert. Es soll untersucht werden, mit welchen Selektionsstrategien die Frequenz der Osteochondrose gesenkt werden kann. Die Auswirkungen auf Leistungsmerkmale durch eine verstärkte Selektion gegen Osteochondrose sind ebenfalls Gegenstand dieser Untersuchung.

Mit Hilfe dieses Simulationsprogramms soll es letztendlich möglich sein, die Auswirkungen von Selektionsstrategien gegen Osteochondrose auf eine Pferdepopulation in kurzer Zeit nachzuvollziehen und damit Empfehlungen an Zuchtverbände geben zu können, wie und in welcher Form die Selektion gegen Osteochondrose in ein Zuchtprogramm integriert werde sollte, um möglichst rasch große Zuchterfolge auf diesem Gebiet erlangen zu können.

\subsection{Einsatzmöglichkeiten der Computersimulation}

Neben der Gewinnung von experimentellen Daten im Rahmen von realen Labor- bzw. Feldversuchen erlangte die Erzeugung sogenannter „synthetischer“ Daten durch die Computer-Simulation in der letzten Zeit immer größere Bedeutung. Die Gründe für die zunehmende Anwendung von Computer-Simulationen sind vielfältig. An erster Stelle ist hier das rasante Wachstum des Leistungsvermögens moderner Computer-Systeme $\mathrm{zu}$ nennen, das dabei Möglichkeiten eröffnet, die früher noch nicht vorstellbar waren. Weiterhin ist die Datengewinnung anhand der Computer-Simulation oft viel kostengünstiger und mit weniger Zeitaufwand verbunden, als die herkömmliche Gewinnung von experimentellen Daten im Rahmen von Labor- bzw. Feldversuchen. Zudem lassen sich Computer-Experimente unter gleich bleibenden Versuchsbedingungen beliebig oft wiederholen, was bei Laborbzw. Feldversuchen zum Teil größere Probleme bereitet. 
Die Computersimulation, auch als Monte-Carlo Methode bezeichnet, wurde von FRASER 1957 in die Genetik eingeführt und ist seitdem für viele populationsgenetische Untersuchungen angewendet worden. Anhand einer simulierten Population können die Auswirkungen verschiedener Selektionsmethoden auf unterschiedliche Parameter nachvollzogen werden. Die Untersuchungen dieser Studie könnten entweder theoretisch, per Simulation oder an realen Daten durchgeführt werden. Simulationsmethoden sind einfacher anzuwenden als theoretische Ableitungen, erfordern aber einen relativ hohen Computeraufwand. Vor allem bietet die Computersimulation den Vorteil, Änderungen der Populationsstruktur und der Parameter untersuchen zu können.

BRUNS und GLODEK (1976) benutzten die Methode der Monte-Carlo Computersimulation für den Vergleich verschiedener Selektionsmethoden. FAMULA und VON FLECK (1982) untersuchten mit dieser Methode den Einfluss verschiedener Selektionsmethoden auf unterschiedliche genetische Gruppierungen bei der Leistungsprüfung von Bullen. SWALVE und BRUNS (1986) überprüften den Effekt der Gruppierung von Bullen bei der Zuchtwertschätzung nach der BLUP Methode. Mit Hilfe der Monte-Carlo Simulation war es möglich, spezielle Situationen zu untersuchen, die bei der Zuchtwertschätzung in der Milchrinderzucht auftreten können. MEUWISSEN und VAN DER WERF (1993) beurteilten den Effekt der Beeinflussung heterogener Varianzen auf genetische Fortschritte und auf Regressionen wahrer Zuchtwerte auf geschätzte Zuchtwerte mittels einer Monte Carlo Simulation. DIAZ et al. (1999) verglichen mit dieser Methode den Einfluss verschiedener Selektionsstrategien, die auf die auf die Veränderung zweier, korrelierter Merkmale abzielten, auf eine simulierte Ziegen Population. KARSTEN und KRIETER (2000) simulierten in ihrer Studie die Verbreitung der Schweinepest in Deutschland. Die Virusausbreitung wurde ausgehend von einem Primärausbruch räumlich und zeitlich zwischen Betrieben in einer abgegrenzten Region simuliert. HAYES und GODDARD (2003) untersuchten die ökonomischen Effekte von DNA Markern, die auf QTLs hinwiesen, im Rahmen einer Markergestützten Selektion anhand einer computersimulierten Schweinepopulation. 
SCHWARZENBACHER et al. (2004) führten eine Simulationsstudie zur Markergestützten Selektion mit komplexem Zuchtziel beim Rind durch. Ziel der Studie war es, durch Simulation von Evolution, Domestikation und systematischer Selektion von vier korrelierten Merkmalen unterschiedlicher Heritabilität möglichst realistische Verteilungen von QTL Effekten und Allelfrequenzen zu erzielen. SULLIVAN et al. (2005) entwickelten genetische Bewertungsstrategien für mehre Merkmale in unterschiedlichen Ländern. Hierzu benutzten sie simuliertes Datenmaterial für drei Laktationsmerkmale aus unterschiedlichen Ländern, die alle typische Nachkommen-Test Programme durchführen.

\subsection{Beschreibung des Simulationsprogramms}

\subsubsection{Erstellung der simulierten Leistungsdaten}

Für die Untersuchung werden die Leistungsdaten von Hengsten und Stuten unter Anwendung der Monte-Carlo-Simulationstechnik erstellt. Die Generierung der Zuchtwerte eines Hengstes oder einer Stute in der Basispopulation kann in Anlehnung an ERIKSSON et al. (1981) und RÖNNINGEN (1970) für die drei gewählten Merkmale Dressur, Springen und Osteochondrose wie folgt abgeleitet werden (BRUNS, 1987):

$$
\left(\begin{array}{l}
a_{i j k l m 1} \\
a_{i j k l m 2} \\
a_{i j k l m 3}
\end{array}\right)=\left(\begin{array}{ccc}
P_{11} & 0 & 0 \\
0 & P_{22} & 0 \\
0 & 0 & P_{33}
\end{array}\right)^{1 / 2} \cdot\left(\begin{array}{ccc}
k_{1} & 0 & 0 \\
k_{2} & k_{3} & 0 \\
k_{4} & k_{5} & k 6
\end{array}\right) \cdot\left(\begin{array}{c}
v_{1} \\
v_{2} \\
v_{3}
\end{array}\right)
$$

$\mathrm{V}_{\mathrm{n}} \quad$ Pseudozufallszahlen aus NID $(0,1)$

$\mathrm{P}_{\mathrm{nn}} \quad$ phänotypische Varianz im n-ten Merkmal

$\mathrm{h}^{2} \quad$ Heritabilität im n-ten Merkmal

$r_{\text {gnm }}$ genetische Korrelationen zwischen dem n-und m-ten Merkmal 
$k_{1}=h_{1}$

$k_{2}=r_{g 1,2} \cdot h_{1} \cdot h_{2} / k_{1}$

$k_{3}=\sqrt{h_{2}^{2}-k_{2}^{2}}$

$k_{4}=r_{g 1,3} \cdot h_{1} \cdot h_{3} / k_{1}$

$k_{5}=\left(r_{g 2,3} \cdot h_{2} \cdot h_{3}-k_{2} \cdot k_{4}\right) / k_{3}$

$k_{6}=\sqrt{h_{3}^{2}-k_{4}^{2}-k_{5}^{2}}$

In den Folgegenerationen werden die Zuchtwerte der Tiere nicht völlig neu generiert, sondern aus einem Teil, der sich hälftig aus dem Vater- und Mutterzuchtwert ergibt, und aus einem zweiten Teil, der nach dem oben gezeigten Prinzip multipliziert mit der Quadratwurzel aus 1/2 erzeugt wird, um die zufällige Kombination der Gene zu simulieren. Die Erstellung der zufälligen Umwelteffekte $\left(\mathrm{e}_{\mathrm{ijk} k \mathrm{~m}}\right)$ erfolgt entsprechend:

$$
\left(\begin{array}{l}
a_{i j k l m 1} \\
a_{i j k l m 2} \\
a_{i j k l m 3}
\end{array}\right)=\left(\begin{array}{ccc}
P_{11} & 0 & 0 \\
0 & P_{22} & 0 \\
0 & 0 & P_{33}
\end{array}\right)^{1 / 2} \cdot\left(\begin{array}{lll}
l_{1} & 0 & 0 \\
l_{2} & l_{3} & 0 \\
l_{4} & l_{5} & l_{6}
\end{array}\right) \cdot\left(\begin{array}{c}
v_{1} \\
v_{2} \\
v_{3}
\end{array}\right)
$$

Hier sind $v_{n}^{a}$ sind Pseudozufallszahlen, unkorreliert mit $v_{n}$ und $l_{\mathrm{o}}$ sind Koeffizienten, die sich aufgrund der umweltbedingten Varianzen und Kovarianzen ergeben.

$l_{1}=e_{1}$

$l_{2}=r_{e 1,2} \cdot e_{1} \cdot e_{2} / l_{1}$

$l_{3}=\sqrt{e_{2}^{2}-l_{2}^{2}}$

$l_{4}=r_{e 1,3} \cdot e_{1} \cdot e_{3} / l_{1}$

$l_{5}=\left(r_{e 2,3} \cdot e_{2} \cdot e_{3}-l_{2} \cdot l_{4}\right) / l_{3}$

$l_{6}=\sqrt{e_{3}^{2}-l_{4}^{2}-l_{5}^{2}}$

$\mathrm{e}^{2}{ }_{\mathrm{n}}=$ relative umweltbedingte Varianz

$\mathrm{r}_{\mathrm{enm}}=$ umweltbedingte Korrelation zwischen dem n-und m-ten Merkmal 


\subsubsection{Erstellung der simulierten Population}

Um mit Hilfe von Simulationsstudien eine möglichst breite und allgemeingültige Aussage zu treffen, sollte die simulierte Population sich in der Struktur und Dynamik den realen Populationen, für die letztendlich die Aussagen gelten sollen, weitestgehend anpassen (BRUNS, 1987).

Die erste Generation (Basisgeneration) wird in Anlehnung an Verhältnisse aus der praktischen Pferdezucht erstellt und besteht aus 5000 Zuchtstuten und 100 Zuchthengsten. Mittels Zufallspaarung entsteht daraus die erste Generation mit dem Ziel, die gewünschte Familienstruktur zu schaffen. Ein Hengst wird an 200 zufällig ausgewählte Stuten angepaart. Eine Stute produziert innerhalb einer Generation vier Fohlen, zwei Stut- und zwei Hengstfohlen. Innerhalb einer Generation, die in Anlehnung an reale Verhältnisse aus der Pferdezuchtpraxis einem Zeitraum von sechs Jahren entspricht, werden somit von 5000 Stuten 20.000 Fohlen beiderlei Geschlechts hervorgebracht. Aus dieser Population werden die Elterntiere der nächsten Generation nach einem zu definierenden Selektionsindex selektiert. Es handelt sich hier also nicht um überlappende Generationen.

Um die Population in der angegebenen Größe konstant $\mathrm{zu}$ halten, kommen Gesamtremontierungsraten zum Einsatz. Die Selektionsschärfe bei Stuten und Hengsten der simulierten Population ergibt sich aus dem Verhältnis der Anzahl der Zuchthengste und Zuchtstuten zur Anzahl der Stut- und Hengstfohlen. Für die Zuchtstuten wird eine Gesamtremontierungsrate von 50\% und für die Zuchthengste von $1 \%$ angesetzt. Das bedeutet, dass die Hälfte der erzeugten Stutfohlen als Zuchtstuten eingesetzt wird. Von den 10.000 erzeugten Hengstfohlen werden jedoch nur 100 für die Körung ausgewählt und kommen als mögliche Vatertiere in Betracht. Die Selektion auf der Hengstseite ist somit um ein vielfaches schärfer als auf der Stutenseite, was der Selektion in der Pferdezuchtpraxis entspricht. Insgesamt werden bis zu fünf Generationen und beliebig viele Wiederholungen erzeugt. Die Ergebnisse wurden unter Einbeziehung von 50 Wiederholungen berechnet. 


\subsubsection{Parameter der simulierten Population}

Die für die Erstellung der simulierten Populationen benutzten Parameter sollten weitgehend den wahren Populationsparametern entsprechen, um zu möglichst aussagekräftigen und auf die Realität übertragbaren Ergebnissen zu gelangen. Diese Maßgabe wurde hinsichtlich der gewählten Parameter für die Größe, der Fruchtbarkeitssituation und der OC-Frequenz der betrachteten Population berücksichtigt. Die Population besteht aus 100 Zuchthengsten und 5000 Zuchtstuten. Diese Stuten erzeugen innerhalb einer Generation, die einem Zeitraum von sechs Jahren entspricht, insgesamt vier Fohlen. Das bedeutet, dass jede Stute in sechs aktiven Zuchtjahren vier effektive Zuchtjahre aufweisen kann. Diese Konstellation orientiert sich an der Fruchtbarkeitssituation in der Praxis, in der eine Trächtigkeitsrate von 60-65\% als eine gute Fortpflanzungsleistung angesehen wird (DOHMS, 2002). Auch UPHAUS (1989) fand für die Hannoveraner Population eine mittlere Abfohlrate von 57,7\%. Für Stuten eines ausgewählten Geburtsjahrganges (1977) stellte er eine mittlere Nutzungsdauer von 4,7 Jahren fest, in der die Stuten durchschnittlich 2,7 Fohlen zur Welt brachten.

Die Angabe der OC-Frequenz innerhalb der simulierten Population orientierte sich an den Ergebnissen diverser Studien, die sich mit der Schätzung der OC-Frequenz in verschiedenen Populationen beschäftigt haben. BARNEVELD und VAN WEEREN (1999a) ermittelten eine Gesamtfrequenz osteochondrotischer Veränderungen an der Lokalisation Sprung- und/oder Kniegelenk im Bereich von 20-26\% bei holländischen Warmblutpferden. Untersuchungen von BREHM und STAECKER (2000) an 1190 Trabrennpferden ergaben eine Befundhäufigkeit von 12,4\% im Sprunggelenk. In ihrer Untersuchung an 733 Pferden der Rasse Selle Français konnten RICARD et al. (2002) ein deutliches Auftreten osteochondrotischer Veränderungen an den verschiedenen Lokalisationen nachweisen. Frequenzen von 19\% im Fessel-, 24\% im Sprung- und 8\% im Kniegelenk wurden hier nachgewiesen. ARNAN (2003) ermittelte anhand der Analyse der Röntgenbilder von 630 hannoverschen Fohlen und deren Müttern; sowie einer weiteren röntgenologischen Untersuchung von 405 derselben Fohlen im Alter von zwei Jahren folgende Ergebnisse: (32,6\%) und 106 Zweijährige (26,2\%) waren positiv. 
Das Fesselgelenk war das am häufigsten betroffene Gelenk, 19,5\% der Fohlen und 16,6\% der Zweijährigen zeigten hier positive Befunde. Gefolgt vom Sprunggelenk, hier fanden sich eine Osteochondrose Frequenz von 11,1\% bei den Fohlen und 10,4\% bei den Zweijährigen. Das Knie erwies sich als das am wenigsten betroffene Gelenk mit 7,2\% der Fohlen und 2,2\% der Zweijährigen. Ergebnisse von WITTWER et al. (2004) an 118 süddeutschen Kaltblutfohlen zeigten eine Frequenz von röntgenologischen Veränderungen, die sich dem Osteochondrose Komplex zuordnen lassen, von 59\%. Von diesen 118 Fohlen zeigten 61,8\% (73 Fohlen) röntgenologische Verschattungen. Zusammenfassend lässt sich sagen, dass die Frequenz der Osteochondrose bei verschiedenen Rassen in einem Bereich von etwa 10-30\% liegt (BRUNS, 2001). Ausgehend von diesen Ergebnissen wurde für die simulierte Population eine Genotypfrequenz des Osteochondrose Befalls von 25\% angegeben.

In der Basispopulation wurde der OC-Befall zufällig mittels des Zufallszahlengenerators verteilt.

\subsection{Entwicklung der genetischen Modelle}

Um die mögliche Vererbung und das genetische Modell für Osteochondrose zu simulieren, wurden zwei genetische Modelle für OC entwickelt. Da die genaue Vererbung der Erkrankung noch nicht bekannt ist, können diese genetischen Modelle lediglich als eine Annahme über die Vererbung angesehen werden.

Im genetischen Modell I wird OC als ein Genlocus mit zwei Allelen angenommen, bei dem OC durch einen autosomal rezessiven Erbgang vererbt wird. Die Genotypen für $\mathrm{OC}$ sehen folgendermaßen aus: $\mathrm{aa}=\mathrm{OC}$ befallen, $\mathrm{Aa}$ und $\mathrm{AA}=\mathrm{OC}$ frei. Die homozygot befallenen Pferde mit dem Genotyp aa (und die OC freien Pferde mit dem Genotyp AA) werden durch die Diagnose anhand eines Röntgenbilds als eindeutig befallen (oder nicht befallen) angesehen. Die heterozygot befallenen Pferde mit dem Genotyp Aa werden auch den nicht befallenen Pferde zugeordnet und somit als OC frei eingestuft. Das heißt, obwohl die Pferde mit einem für OC heterozygotem Genotyp OC-befallen sein können, werden sie anhand ihres Phänotyps für OC (Röntgenbild) den nicht befallenen Pferden zugeordnet. In diesem Modell entspricht der Genotyp also dem Phänotyp. 
In beiden genetischen Modellen wird die Selektion auf Basis der Beurteilung von Röntgenbildern simuliert. Die Diagnose anhand von Röntgenbildern kann durch Diagnosefehler, mangelhafte Röntgenbilder etc., ungenau sein, dieses Szenario wird durch die Angabe einer Penetranz für das Merkmal OC simuliert. Die Penetranz eines Merkmals ist der Anteil der Individuen einer Population, die einen homozygoten Genotyp für das Merkmal aufweisen, bei denen der Befall aber nicht festgestellt werden kann. Die Penetranz wird mit $80 \%$ angegeben, das heißt, dass von 100 befallenen Pferden bei 20 Pferden der Osteochondrose-Befund nicht entdeckt wird.

Das Programm erlaubt die Generierung von drei verschiedenen Merkmalen: es wurden zwei Leistungsmerkmale (Dressur und Springen) und ein Krankheitsmerkmal (OC) gewählt, für die jeweils der Geno- und Phänotypwert erzeugt wird. Für die beiden Leistungsmerkmale wurde für den Geno- und Phänotyp jeweils eine Normalverteilung mit statistischen Kennzahlen gewählt.

In der Basispopulation sehen die Häufigkeitsverteilungen und Parameter wie folgt aus:

\section{Leistungsmerkmal 1 („Dressurveranlagung“):}

Genotyp gleich Zuchtwert, Mittel=0, Varianz= Heritabilität · phänotypische Varianz, Phänotyp, Mittel=0, Varianz=1, normalverteilt

\section{Leistungsmerkmal 2 ( „Springveranlagung“):}

Genotyp gleich Zuchtwert, Mittel=0, Varianz= Heritabilität $\cdot$ phänotypische Varianz, Phänotyp, Mittel=0, Varianz=1, normalverteilt

Es wurden zwei genetische Modelle für OC entwickelt, die sich folgendermaßen darstellen:

\section{Gesundheitsmerkmal (Osteochondrose) genetisches Modell I:}

Genlocus mit zwei Allelen, drei Genotypen ( $a=\mathrm{aC}$ befallen, Frequenz $=\mathrm{p}^{2}$, Aa und AA nicht befallen), Phänotyp eingeteilt in zwei Klassen (Phänotypwert=0, d.h. OC festgestellt, Phänotypwert=1, d.h. OC nicht festgestellt= alle Aa und AA-Genotypen zuzüglich die nicht als befallen identifizierten aa-Genotypen) 


\section{Gesundheitsmerkmal (Osteochondrose) genetisches Modell II:}

Genotyp gleich Zuchtwert, Mittel=0, Varianz= Heritabilität · phänotypische Varianz, Phänotyp, Mittel=0, Varianz $=1$, normalverteilt

Phänotypwert=0, d.h. OC festgestellt, Häufigkeit entsprechend der vergebenen

Frequenz unterhalb der Festgrenze

Phänotypwert=1, d.h. OC nicht festgestellt

Für alle Variablen müssen deren phänotypische und genetische Varianzen und Kovarianzen angegeben werden; die Mittel werden in der Basispopulation auf null gesetzt.

Im zweiten genetischen Modell ist Osteochondrose kein Genort, sondern ein phänotypisches Ja-oder-Nein Merkmal mit einer zugrunde gelegten genetischen Normalverteilung. Aufgrund der Frequenz von OC wird eine Einteilung in phänotypische Klassen vorgenommen. Die Grenzen für die Klassen werden durch eine Festgrenze definiert, die den Phänotyp für OC Befall in zwei Klassen teilt, OC positiv und OC negativ. Unterhalb dieser Grenze sind die Pferde OC positiv (Wert 0), oberhalb der Grenze sind die Pferde OC-negativ (Wert 1); diese Festgrenze bleibt auch für weitere Generationen fix. Auch in diesem Modell erfolgt die Angabe einer Penetranz für das Merkmal OC in Höhe von $80 \%$. Da hier die Annahme erfolgt, dass OC, wie Dressur und Springen ein phänotypisches, normalverteiltes Merkmal ist, könnten in diesem Modell Korrelationen und Umwelteffekten zwischen Osteochondrose und Leistungsmerkmalen angegeben werden. 


\subsubsection{Die Penetranz}

Die in den beiden genetischen Modellen verwendete Penetranz soll hier noch einmal näher erläutert werden. TIMOFEFF-RESSOVSKY (1979) bezeichnete die Penetranz als den Anteil eines Genotyps, der das betreffende Merkmal manifestiert. Unvollständige Penetranz (w), z.B. des homozygot Rezessiven, hat folgende Verteilung der Phänotypen zur Folge:

$$
\begin{array}{cccc}
\text { Genotyp } & \text { AA } & \text { Aa } & \text { aa } \\
\text { Frequenz } & \frac{p^{2}}{1-w q^{2}} & \frac{2 p q}{1-w q^{2}} & \frac{p q^{2}}{1-w q^{2}}
\end{array}
$$

Die Wahrscheinlichkeit, dass ein Tier ohne Träger des Merkmals zu sein, den Genotyp aa hat, beträgt:

$$
\frac{(1-w) q^{2}}{1-w q^{2}}
$$

Untersuchungen haben ergeben, dass das Syndrom der Arthrogrypose und Palatoschisis (SAP, Anzeichen der Krankheit sind Gaumenspalten und versteifte Gelenke) in der Rasse Charolais durch ein rezessives Gen mit einer Penetranz von weniger als $1 / 4$ verursacht wird, d.h., dass weniger als $1 / 4$ der rezessiv homozygoten den Fehler auch zeigen (PIRCHNER, 1979). Um in dem hier entwickelten Computerprogramm die Penetranz des OC- Befalls zu simulieren, wurde von den OC-befallenen Pferden der Anteil (1-w) als gesund angegeben. Die OCGenotypfrequenz beträgt in der Basispopulation 0,25. Durch die Anwendung der Penetranz ergab sich eine phänotypische OC-Frequenz der Basispopulation von 0,2, deren Veränderungen bei der Ergebnisfindung betrachtet wurden. 


\subsection{Entwicklung der Selektionsmethoden}

Um verschiedene Selektionsstrategien $\mathrm{zu}$ simulieren, wurden zwei Selektionsmethoden entwickelt: die einstufige und die zweistufige Selektion. Bei der einstufigen Selektion vollzieht sich die Selektion für alle drei Merkmale innerhalb einer Selektionsstufe. Bei dieser Methode wird die gesamte Population (Hengste und Stuten) auf einen möglichen Osteochondrose-Befall hin röntgenologisch untersucht. Bei der zweistufigen Selektionsmethode verläuft die Selektion auf zwei Stufen. Auf der ersten Selektionsstufe werden die Hengste aufgrund ihrer phänotypischen Leistungen in der Dressur und im Springen ausgewählt. Auf der zweiten Selektionsstufe wird nach einem Selektionsindex selektiert, in dem Dressur, Springen und Osteochondrose mit den in Tabelle 6 dargestellten Selektionsgewichten gewichtet wurden. Dieses Selektionsszenario soll das Auswahlverfahren für Hengste im Rahmen der Körung aus der Praxis simulieren (s. Kapitel Hengstselektion). Die erste Selektionsstufe stellt den Vorauswahltermin bei der Hengstkörung dar. Hier werden ca. 700 Hengste vorgestellt und auf ihre Leistungen in den Disziplinen Dressur und Springen überprüft. Hengste mit überragenden Leistungen werden zur Körung zugelassen, die bei der zweiten Selektionsstufe simuliert wird. Bei der Körung findet eine nochmalige Überprüfung der Hengste in den Leistungsmerkmalen Dressur und Springen sowie eine Überprüfung hinsichtlich des röntgenologischen Status des Hengstes statt. Dieses Auswahlverfahren wird in der Praxis nur bei den Hengsten einer Population angewendet. Um eine möglichst realistische Simulation und eine Übertragbarkeit der Ergebnisse auf die Realität zu gewährleisten, werden bei den verschiedenen zweistufigen Selektionsszenarien nur die Hengste der simulierten Population in die Selektion gegen Osteochondrose einbezogen. Die Stuten werden hier lediglich einer Selektion in den Merkmalen Dressur und Springen unterzogen. 


\subsection{Simulation verschiedener Selektionsszenarien gegen OC aus der Pferdezuchtpraxis}

Mit Hilfe der vorgestellten genetischen Modelle und Selektionsmethoden konnten mögliche Selektionsszenarien zur Reduzierung der Osteochondrose Frequenz simuliert werden, wie z.B. das Vorhandensein eines Gentests gegen die Osteochondrose oder die verstärkte Einbeziehung der Stuten in die Selektion gegen Osteochondrose. Außerdem war es durch eine Weiterentwicklung des zweistufigen genetischen Modells II mit zwei Klassen möglich, Selektionsszenarien zu simulieren, die bei der Hengstkörung Anwendung finden. Die Auswirkungen von Selektionsverfahren auf der Hengstseite einer Population wurden besonders intensiv untersucht, da sich züchterische Maßnahmen in traditionellen Zuchtprogrammen der Warmblutzucht vor allem auf die Hengste konzentrieren (BRUNS, 2005). Dies ist darauf zurückzuführen, dass Zuchthengste aufgrund der Anzahl ihrer Nachkommen, die von ihnen produziert werden können (vor allem seit dem vermehrten Einsatz der künstlichen Besamung in der Pferdezucht), einen größeren Einfluss auf Zuchtentwicklungen nehmen können, als die Stutenseite.

\subsubsection{Selektionsszenarien bei der Körung}

Beim genetischen Modell II mit zwei phänotypischen Klassen wird OC als ein Jaoder-Nein Merkmal mit einer zugrundegelegten Normalverteilung definiert, es liegt entweder ein OC-Befall vor oder das Pferd ist OC frei. Diese Einteilung wird aufgrund der Frequenz der OC innerhalb der Population vorgenommen. Anhand der Frequenz wird eine Festgrenze definiert, die den Phänotyp in zwei Klassen OCpositiv oder OC-negativ einteilt. Dieses genetische Modell II diente als Basismodell für die Entwicklung weiterer Selektionsmodelle, bei denen OC ebenfalls als ein normalverteiltes Merkmal angenommen wurde.

Das genetische Modell II mit vier phänotypischen Klassen stellt eine Weiterentwicklung des Modells mit zwei Klassen dar, hier wird der Phänotyp in vier Klassen eingeteilt, die einer Einteilung von Röntgenbefunden in das 1993 von der internationalen Röntgenkommission entwickelte Bewertungsschema mit vier Klassen nachempfunden sind. 
Bei diesem Modell erfolgt eine Indexselektion anhand der phänotypischen Leistung in Dressur, Springen und OC mit einer Gewichtung, die den Heritabilitäten der Merkmale entspricht; weiterhin erfolgt eine Zuchtwertschätzung für die drei Merkmale anhand der Eigenleistung eines Hengstes. Die vier Röntgenbewertungsklassen dienen als Maßstab für tierärztliche Diagnosen, um Röntgenbefunde nach ihrem Schweregrad abstufen und einteilen zu können. In die Röntgenklasse eins werden Pferde eingeteilt, die röntgenologisch keinen besonderen Befund aufweisen, die Röntgenklasse zwei beinhaltet Befunde, die geringgradig von der Norm abweichen. In die Röntgenklassen drei und vier werden röntgenologische Befunde eingeteilt, die deutlich von der Norm abweichen (Klasse drei) oder sehr wahrscheinlich zu Beeinträchtigungen führen (Klasse vier). Die folgende Abbildung 3 verdeutlicht die Einteilung des Phänotyps in vier Klassen:

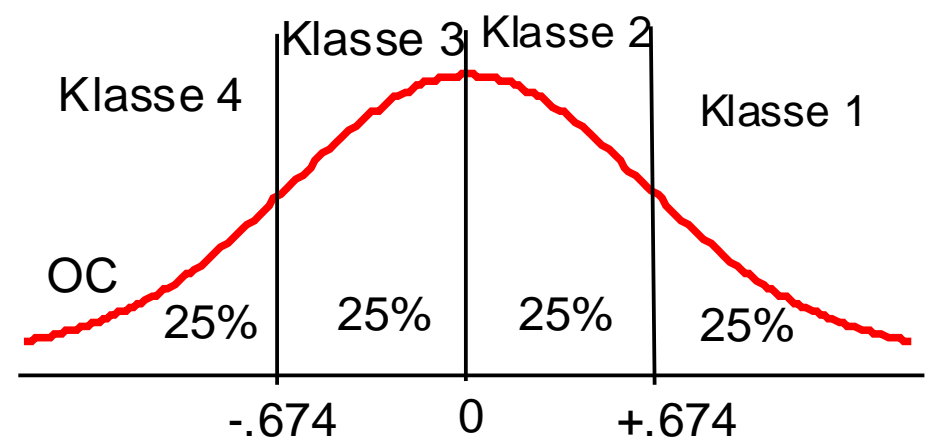

Abbildung 3: Einteilung des normalverteilten Phänotyps in vier Klassen bei genetischem Modell II mit vier Klassen

Die Einteilungsgrenzen der einzelnen vier Klassen werden aufgrund der Frequenz der Osteochondrose definiert. In der simulierten Population wird eine OCGenotypfrequenz Frequenz von 25\% angenommen. Die Frequenz 25\% entspricht bei der Normalverteilungstabelle einem Grenzwert von 0,674, der im positiven und negativen Bereich die Grenze für die Einteilung in die jeweiligen Klassen darstellt. Die zweistufige Selektion wird bei diesem Modell fortgeführt. Hier können die Hengste auf der zweiten Selektionsstufe, welche die Körung simuliert, durch eine überdurchschnittliche Leistung in Dressur und Springen eine Einteilung in die Röntgenklasse drei oder vier ausgleichen und trotzdem noch ein positives Körurteil erzielen. 
Dieses Modell mit vier Klassen diente wiederum als Basis für die Entwicklung weiterer Selektionsmodelle, die das Auswahlverfahren bei der Körung simulieren. Die so entwickelten Modelle weisen ebenfalls, wie dieses Ausgangsmodell, vier phänotypische Klassen auf. Zum Vergleich der Ergebnisse und der erzielten Selektionserfolge werden die Modelle im weiteren Verlauf dieser Arbeit mit den Bezeichnungen „Modell mit vier Klassen“, „Ausschlussmodell(e)“ und „Zuchtwertschätzmodell“ benannt.

Ein weiteres in der Praxis angewandtes Selektionsverfahren bei der Körung ist der Ausschluss von Hengsten, die aufgrund ihrer röntgenologischen Befunde in die Röntgenklasse drei und/oder vier eingeteilt worden sind. Mit der Maßgabe, dieses Selektionsszenario zu simulieren, wurden auf Basis des Selektionsmodells mit vier Klassen zwei weitere Ausschlussselektionsmodelle entwickelt. Bei diesen Modellen findet keine Zuchtwertschätzung statt, sondern ein Ausschluss von Hengsten aufgrund ihres röntgenologischen Status. Auf der ersten Selektionsstufe wird aufgrund der Leistungen der Hengste in Dressur und Springen selektiert. Auf der zweiten Selektionsstufe werden bei der nochmaligen Überprüfung der Dressur- und Springleistung sowie der zusätzlichen Überprüfung des OC-Status, alle Hengste, die bezüglich ihrer röntgenologischen Befunde im Hinblick auf OC eine Röntgenklasse vier aufweisen, von der Zucht ausgeschlossen. Bei dem zweiten Ausschlussselektionsmodell wird an diesem Punkt schärfer selektiert, hier werden alle Hengste mit einer Röntgenklasse drei und vier von der Zucht ausgeschlossen. Im Vergleich zum Modell mit vier Klassen können überdurchschnittliche Leistungen der Hengste in den Merkmalen Dressur und Springen bei den Ausschlussmodellen kein Ausgleich für eine Einteilung ihrer Röntgenbefunde in die Klassen vier/ drei und vier sein, da diese Hengste von weiteren Selektionsentscheidungen ausgeschlossen werden. 
Eine Zuchtwertschätzung für OC würde die Möglichkeit bieten, bereits zum Zeitpunkt der Körung eines Junghengstes Informationen über seinen OC-Status in Form eines Zuchtwertes vorliegen zu haben. Dies würde einen großen zusätzlichen Informationsgewinn für die Körkommission und natürlich auch für die Hengstbesitzer und Züchter, die den Junghengst möglicherweise in Zukunft züchterisch nutzen möchten, darstellen; vor allem, wenn der OC-Status von verwandten Pferden, z.B. Halbgeschwistern zusätzlich vorliegen würde. Um dieses Szenario zu simulieren, wurde auf der Basis des genetischen Modells II mit vier phänotypischen Klassen eine Zuchtwertschätzung mit einem zugrundegelegten Tiermodell für das Merkmal Osteochondrose in das Simulationsprogramm eingebaut. Die Selektion erfolgt nach geschätzten Zuchtwerten, die sich aus der Eigenleistung in Dressur, Springen und OC sowie aus der Halbgeschwisterleistung in den entsprechenden Merkmalen ergeben. Die Berechnung erfolgte hier nicht durch ein vollständiges Tiermodell, sondern durch ein Tiermodell mit alleiniger Berücksichtigung der väterlichen Verwandtschaft. Die mütterliche Verwandtschaft wird eher als unbedeutend eingestuft, da mehr Nachkommen eines Hengstes als einer einzelnen Stute existieren. Dieses Modell beruht also nicht wie alle anderen Selektionsmodelle ausschließlich auf der Eigenleistung des Probanden, sondern hier werden die Leistungen der väterlichen Halbgeschwister einbezogen. Die zweistufige Selektion bleibt bestehen wie oben dargestellt. Die Anzahl Halbgeschwister variiert von Pferd $\mathrm{zu}$ Pferd, sie liegt zwischen eins und der maximalen Anzahl Halbgeschwister. 
Tabelle 5 gibt einen Überblick über die entwickelten genetischen Modelle und Selektionsmethoden:

Tabelle 5: Überblick über entwickelte genetische Modelle und Selektionsmethoden

\begin{tabular}{|l|l|}
\hline Selektionsmethode & \multicolumn{1}{|c|}{ Genetisches Modell } \\
\hline Einstufige Selektion & Modell I: OC als Genort \\
\cline { 2 - 3 } & $\begin{array}{l}\text { Modell II: OC als normalverteiltes Merkmal mit zwei } \\
\text { phänotypischen Klassen }\end{array}$ \\
\hline Zweistufige Selektion & Model I: OC als Genort \\
\cline { 2 - 3 } & $\begin{array}{l}\text { Modell II: OC als normalverteiltes Merkmal mit zwei } \\
\text { phänotypischen Klassen }\end{array}$ \\
\hline Zweistufige Selektion & $\begin{array}{l}\text { Modell II: OC als normalverteiltes Merkmal mit vier } \\
\text { phänotypischen Klassen }\end{array}$ \\
\cline { 2 - 3 } & $\begin{array}{l}\text { Modell II: OC als normalverteiltes Merkmal mit vier } \\
\text { phänotypischen Klassen, Röntgenklasse vier gemerzt }\end{array}$ \\
\cline { 2 - 3 } & $\begin{array}{l}\text { Modell II: OC als normalverteiltes Merkmal mit vier } \\
\text { phänotypischen Klassen, Röntgenklassen drei+vier gemerzt }\end{array}$ \\
\cline { 2 - 3 } & $\begin{array}{l}\text { Modell II: OC als normalverteiltes Merkmal mit vier } \\
\text { phänotypischen Klassen, Zuchtwertschätzung für OC }\end{array}$ \\
\hline
\end{tabular}

\subsubsection{Weitere simulierte Selektionsszenarien}

Die in der Zukunft eventuelle mögliche Diagnose der OC anhand eines Gentests für OC stellt ein weiteres mögliches Selektionsszenario dar. Dies konnte innerhalb der zweistufigen Selektion durch die Gewichtung des Genotyps für OC simuliert werden.

In den oben vorgestellten Auswahlszenarien bei der Körung findet keine Einbeziehung der Stuten in die Selektion statt. Verschiedene Studien haben jedoch herausgefunden, dass durch die zusätzliche Einbeziehung der Stutenseite in die Selektion deutliche Erfolge bei der Reduktion der OC-Frequenz erzielt werden können. Aus diesem Grund wurde dieses Szenario durch den Vergleich zwischen der einstufigen Selektion nur der Hengste (hier wurde das Selektionsgewicht für OC auf der Stutenseite mit Null angegeben) und der ursprünglichen einstufigen Selektion der Hengste und der Stuten simuliert. 
Diese beiden Selektionsszenarien konnten durch Veränderungen bei den Gewichtungen des OC Merkmals simuliert werden. Es mussten keine Veränderungen des Ausgangssimulationsprogramms vorgenommen werden, so dass sie nicht als eigene genetische Modelle gewertet und deshalb nicht in die obige Tabelle aufgenommen wurden.

\subsection{Erstellung des Selektionsindexes}

Bei der gleichzeitigen Selektion auf mehrere Merkmale gibt es verschiedene Selektionsvarianten, um eine gleichzeitige Leistungssteigerung in allen Merkmalen zu erreichen (KÜNZI und STRANZINGER, 1992):

1) Tandemselektion- In jeder Generation wird auf ein anderes Merkmal selektiert. Dies wird zyklisch wiederholt, bis alle Merkmale an der Reihe waren.

2) Selektion nach unabhängigen Selektionsgrenzen- Für jedes Merkmal wird eine untere Selektionsgrenze festgelegt. Je mehr Merkmale berücksichtigt werden, desto tiefer muss die Selektionsgrenze im einzelnen Merkmal festgelegt werden.

3) Selektion nach abhängigen Selektionsgrenzen oder IndexselektionHAZEL und LUSH (1942) haben gezeigt, dass dieses Selektionsverfahren allen anderen Selektionsverfahren überlegen ist, vor allem bei ungünstigen genetischen Beziehungen zwischen den Merkmalen.

Unter einer Indexkonstruktion verstehen SCHÜLER et al. (2001) die Berechnung der Gewichtungsfaktoren im Index für eine gegebene Kombination von Informationsquellen. Das Ziel der Indexkonstruktion ist die Maximierung der Korrelation zwischen dem wahren und dem geschätzten Zuchtwert. 
Der zur Verbesserung des Gesamtzuchtwertes konstruierte Index lautet (FALCONER, 1984):

$\mathrm{I}=\mathrm{b}_{1} \mathrm{P}_{1}+\mathrm{b}_{2} \mathrm{P}_{2}+\ldots \ldots \mathrm{b}_{\mathrm{m}} \mathrm{P}_{\mathrm{M}}$

$\mathrm{P}_{1}$ bis $\mathrm{P}_{\mathrm{m}}$ stellen die phänotypischen Messungen an den $\mathrm{m}$ Selektionsmerkmalen und $\mathrm{b}_{1}$ bis $\mathrm{b}_{\mathrm{m}}$ die dazugehörigen Gewichtungsfaktoren dar.

Der Gesamtzuchtwert ist definiert als:

$\mathrm{T}=\mathrm{a}_{1} \mathrm{~T}_{1}+\mathrm{a}_{2} \mathrm{~T}_{2}+\ldots . \mathrm{a}_{\mathrm{n}} \mathrm{T}_{\mathrm{n}}$

und ist eine lineare Kombination der mit den relativen ökonomischen Gewichten $\left(\mathrm{a}_{\mathrm{i}}\right)$ multiplizierten Zuchtwerten in den einzelnen Merkmalen $\left(\mathrm{T}_{\mathrm{i}}\right)$.

Die Berechnung der Indexgewichte $b_{i}$ erfolgt unter Maximierung der Beziehung zwischen Index und Gesamtzuchtwert, so dass in Matrixschreibweise folgendes Gleichungssystem aufgestellt werden kann:

$P \cdot b=G \cdot a$

$\mathrm{P}=$ Matrix der phänotypischen Varianzen und Kovarianzen der n Indexmerkmale $\mathrm{b}=$ Spaltenvektor der Indexgewichte

$\mathrm{G}=$ Matrix der genetischen Kovarianzen zwischen den $\mathrm{n}$ Index- und den $\mathrm{m}$

Zuchtwertmerkmalen

$\mathrm{a}=$ Spaltenvektor der relativen ökonomischen Gewichte

Die Lösung für die Indexgewichte ergibt sich nach:

$\mathrm{b}=\mathrm{P}^{-1} \cdot \mathrm{G} \cdot \mathrm{a}$

wobei $\mathrm{P}^{-1}$ die Inverse der Matrix von $\mathrm{P}$ ist.

Der Selektionsindex, der innerhalb dieses Simulationsprogramms eingesetzt wird, kombiniert die phänotypischen und/oder die additiv genetischen Effekte der Merkmale. Der Index wird gebildet, in dem die Phänotyp- und/oder Zuchtwerte mit ihren Gewichtungen multipliziert werden. Die Selektion kann für Stuten und Hengste sowohl in den Selektionskriterien als auch in der Selektionsschärfe unterschiedlich sein. 
Die Indexselektion, bei der eine Berücksichtigung mehrer Merkmale möglich ist, wird zur Verbesserung des ökonomischen Wertes von Tieren oder Pflanzen angewendet, da der ökonomische Gesamtwert von mehr als einem Merkmal abhängt (FALCONER, 1984). Es gibt in der Tierzucht jedoch auch Merkmale, für die ein ökonomischer Gesamtwert, oder ein ökonomisches Gewicht nicht bestimmt werden kann, wie z.B. die Fleischqualität in der Schweinezucht: von wenigen Ausnahmen abgesehen, wird Fleischqualität in Deutschland nicht bezahlt, es gibt daher keinen Grenznutzen für eine verbesserte Fleischqualität (SCHÜLER et al., 2001). Auch für fast alle Merkmale in der Reitpferdezucht lassen sich keine ökonomischen Gewichte ermitteln. In Ermangelung der Kenntnis der ökonomischen Gewichte wurde deshalb bei der Ableitung der optimalen Indexgewichte von einer gleichen ökonomischen Gewichtung der Leistungsmerkmale (Dressur und Springen) und des Merkmals Osteochondrose ausgegangen.

\subsubsection{Die Selektionsgewichte}

Korrelationen zwischen orthopädischen Befunden und den gängigen Selektionskriterien sind für züchterische Fragen relevant, da sie mitunter die tatsächliche Bedeutung der OC für ein Zuchtprogramm umreißen und Auskunft darüber geben, ob eine verstärkte Selektion auf bestimmte Parameter negative Auswirkungen auf die Gliedmaßengesundheit in der Population hat. Es wurden zahlreiche Studien durchgeführt, um die Beziehung zwischen Gliedmaßenerkrankungen und den Kriterien der Leistung beim Pferd zu analysieren, diese Studien haben ergeben, dass keine genetischen Korrelationen zwischen OC und den Leistungsmerkmalen bestehen.

Bei unkorrelierten, unabhängigen Merkmalen, die in einen Selektionsindex einbezogen werden, sollte die Gewichtung der Merkmale anhand der Heritabilitäten der Merkmale erfolgen. Um eine korrekte Gewichtung der ausgewählten Merkmale innerhalb des Selektionsindexes zu gewährleisten, wurden die Ergebnisse verschiedener Heritabilitätsschätzungen herangezogen. Abweichend davon sind die Selektionsgewichte bei dem Selektionsszenario mit Zuchtwertschätzung variabel und abhängig von den vorhandenen Verwandtenleistungen. 
Da in dieser Studie vor allem die Auswirkungen der Selektion auf der Hengstseite einer Population untersucht werden sollen, wurden Schätzwerte einbezogen, die anhand der Daten der stationären Hengstleistungsprüfung berechnet wurden.

Die Publikation von THOREN et al. (2005) gibt einen Überblick über aktuelle Heritabilitätsschätzungen. SCHADE (1996) nutzte Daten von Hengstleistungsprüfungen hannoverscher Hengste. Er schätzte für die Merkmale Grundgangarten, Springen und Rittigkeit Heritabilitäten von 0,38, 0,46 und 0,29. VELSEN-ZERWECK (1998) und BROCKMANN (1998) nutzten Daten der acht Hengstleitungsprüfungsstationen aus den Jahren 186 bis 1995 im Rahmen einer multivariaten Analyse. Die von VELSEN-ZERWECK (1998) geschätzten Erblichkeiten waren geringfügig höher als die Ergebnisse von SCHADE (1996) und betrugen 0,47, 0,47 und 0,52 für die Merkmale Grundgangarten, Springen und Rittigkeit. BROCKMANN (1998) kam zu Ergebnissen im gleichen Bereich: 0,41, 0,49 und 0,43 für die gleichen Merkmale. LÜHRS-BEHNCKE et al. (2002) analysierten die Ergebnisse aller in Deutschland getesteten Hengste in den Jahren 1986 und 2001 durch ein Mehrmerkmals-Tiermodell. Es wurden Heritabilitäten von 0,42 für die Grundgangarten, 0,41 für die Rittigkeit und 0,39 für das Springen geschätzt. In zwei schwedischen Studien schätzten GERBER OLSSON et al. (2000) und GELINDER et al. (2001) Heritabilitäten für die Hengstleistungsprüfung von vier-fünfjährigen Hengsten, die sich über einen Zeitraum von acht Tagen erstreckt. Die erste Studie beinhaltete Ergebnisse aus den Jahren 1979-1993, die zweite Studie bezog Ergebnisse aus den Jahren 1979-1999 ein. Die geschätzten Heritabilitäten lagen für das Merkmal Grundgangarten in einem Bereich von 0,28-0,46, für das Merkmal Springen im Bereich von 0,32 bis 0,55. Um eine einheitliche Gewichtung der Leistungsmerkmale zu gewährleisten, wurden in dieser Studie die Merkmale Dressur und Springen mit einem Wert von 0,4 gewichtet.

Die Schätzungen der Heritabilität der Osteochondrose schwanken je nach der angewandten Methodik und der untersuchten Population sowie der Lokalisation zwischen 0,0 und 0,3 (BRUNS, 2001). Diese Einschätzung konnte anhand der Ergebnisse verschiedener Untersuchungen, die sich mit Heritabilitätsschätzungen für OC an unterschiedlichen Lokalisationen befassten, bestätigt werden. 
GROHNDAHL et al. (1993) ermittelten Heritabilitäten für osteochondrotische Veränderungen am Fesselgelenk anhand unterschiedlicher Schätzmodelle. Bei der Anwendung der REML-Methode wurde ein Ergebnis von 0,13 ermittelt, die Zugrundelegung des Schwellenwertmodells führte $\mathrm{zu}$ einem Wert von 0,21. Zu ähnlichen Ergebnissen kamen PHILIPSSON et al. (1993), sie ermittelten einen Heritabilitätsschätzwert von 0,09 mit Hilfe des Schwellenwertmodells, ein Wert von 0,17 konnte anhand eines linearen Modells ermittelt werden. RICARD et al. (2002) schätzten Heritabilitäten für osteochondrotische Veränderungen am Fesselgelenk vorne von 0,21 und am Fesselgelenk hinten von 0,12. Die Ergebnisse von SCHOBER (2003) für osteochondrotische Veränderungen an dieser Lokalisation siedeln sich mit Heritabilitäten von 0,160 bis 0,177 in einem ähnlichen Bereich an. Bezüglich der OC im Sprunggelenk schätzten SCHOUGAARD et al. (1987), PHILIPSSON et al. (1992), GROHNDAHL et al. (1993) und WILLMS (1998) anhand linearer Modelle an verschiedenen Populationen Heritabilitäten im Bereich von 0,19 bis 0,34. Ausgerichtet an der Schwellenwerttheorie ergaben parallel gelagerte Analysen nach Angabe von GROHNDAHL et al. (1993) und WILLMS (1998) Werte zwischen 0,52 und 0,64. Untersuchungen von BREHM und STAECKER (1994), WINTER (1995) und RICARD et al. (2002) ergaben hingegen deutlich niedrigere Heritabilitäten für Veränderungen im Sprunggelenk, die Ergebnisse lagen hier zwischen 0,000 und 0,007. Die Untersuchungen von SCHOBER (2003) ergaben Ergebnisse in einem ähnlichen Bereich zwischen 0,082 und 0,149 .

Bei der vorliegenden Untersuchung findet keine Unterscheidung zwischen den unterschiedlichen Lokalisationen der Osteochondrose statt, es wird lediglich in OCbefallen oder OC-frei unterschieden. Aufgrund der beschriebenen Ergebnisse diverser Heritabilitätsschätzungen für diese Erkrankung wird die Osteochondrose mit einem Wert von 0,2 gewichtet.

Da die Selektion in dieser Untersuchung auf der Diagnose anhand von Röntgenbildern basiert, die eine Bewertung des Phänotyps darstellen, erfolgt die Selektion anhand der Phänotypwerte der Merkmale mit den entsprechenden Gewichten. 
Die Selektionsgewichte der Zuchtstuten und Zuchthengste werden für jedes Geschlecht einzeln angegeben, es können dieselben oder unterschiedliche Selektionsgewichte verwendet werden.

Um möglichst viele verschiedene Selektionsstärken gegen Osteochondrose zu simulieren, fand eine unterschiedliche Gewichtung der OC statt. Die Gewichtung der Leistungsmerkmale bleibt konstant.

Tabelle 6: Verwendete Selektionsgewichte in dieser Untersuchung

\begin{tabular}{|c|c|c|l|}
\hline \multicolumn{3}{|c|}{ Gewichte für: } & \multicolumn{1}{|c|}{ Beschreibung der Selektion } \\
Dressur & Springen & OC & \\
\hline 0,4 & 0,4 & 0,0 & Selektion ohne Berücksichtigung von OC \\
\hline 0,4 & 0,4 & 0,2 & $\begin{array}{l}\text { Selektionsgewichte entsprechen den } \\
\text { Heritabilitäten der Merkmalen }\end{array}$ \\
\hline 0,4 & 0,4 & 0,4 & Etwas erhöhtes Selektionsgewicht auf OC \\
\hline 0,4 & 0,4 & 0,8 & Höheres Selektionsgewicht auf OC \\
\hline
\end{tabular}

\subsubsection{Die Remontierungsraten}

Der zentrale Ansatzpunkt für die Erhöhung der Selektionsintensität ist die Remontierungsrate. Je schärfer selektiert wird, oder je weniger Individuen einer Population die Eltern der nächsten Generation bilden, desto größer wird der Selektionserfolg ausfallen. Aus diesem Grund sollte jeder Züchter bestrebt sein, so scharf wie möglich zu selektieren und nur eine geringe Remontierungsrate bei seinen Selektionsentscheidungen anzusetzen (SCHÜLER et al., 2001).

Bei der einstufigen Selektionsmethode ist die Remontierungsrate auf 0,5 bei den Stuten und 0,01 bei den Hengsten festgesetzt. Die Regulierung der Selektionsschärfe bei den Leistungsmerkmalen und dem Merkmal OC erfolgt bei der zweistufigen Selektionsmethode über die Angabe unterschiedlicher Remontierungsraten auf der ersten Selektionsstufe. Die Remontierungsrate der zweiten Selektionsstufe wird programmintern berechnet, da die Gesamtremontierungsrate für Stuten auf 0,5 und für die Hengste auf 0,01 bei der simulierten Population festgesetzt ist. Auf der ersten Selektionsstufe gibt die Remontierungsrate an, wie scharf in den Merkmalen Dressur und Springen selektiert wird. 
Die Remontierungsrate der zweiten Stufe regelt die Selektionsschärfe für die gleichzeitige Selektion auf Dressur- und Springeignung und auf den OC-Status. Um verschiedene Selektionsschärfen auf den beiden Selektionsstufen zu simulieren, wurden folgende Remontierungsraten eingesetzt:

Tabelle 7: Verwendete Remontierungsraten für die Selektion der Hengste innerhalb der zweistufigen Selektionsmethode

\begin{tabular}{|c|c|}
\hline \multicolumn{2}{|c|}{ Remontierungsrate } \\
Erste Stufe & Zweite Stufe \\
\hline 0,01 & 1 \\
\hline 0,02 & 0,5 \\
\hline 0,05 & 0,2 \\
\hline 0,1 & 0,1 \\
\hline 0,2 & 0,05 \\
\hline 1 & 0,01 \\
\hline
\end{tabular}

Tabelle 7 verdeutlicht die Selektionsschärfe der angewandten Remontierungsraten. Die niedrige Remontierungsrate 0,01 auf der ersten Stufe bedeutet eine scharfe Selektion für die Merkmale Dressur und Springen, die Remontierungsrate 1 auf der zweiten Stufe beinhaltet keine Selektion gegen OC. Diese Konstellation dreht sich bei den nächsten verwendeten Remontierungsraten um, die Remontierungsrate auf der ersten Stufe erhöht sich, was eine weniger starke Selektion für Dressur und Springen mit sich bringt, während die Remontierungsrate auf der zweiten Stufe niedriger wird, so dass hier ein erhöhter Selektionsdruck für das Merkmal OC entsteht.

\subsection{Methoden der statistischen Auswertung}

Das in der Programmiersprache Fortran 77 erstellte Simulationsprogramm gibt für jede der fünf Generationen die OC-Genotypfrequenz, die OC-Genfrequenz und die Phänotypfrequenz sowie die Zuchtwerte für Dressur und Springen als Durchschnitt über die simulierten 50 Wiederholungen aus. Bei der Ergebnisfindung werden die Parameter aus der fünften Generation betrachtet. 
Für diese Parameter wird die Varianz aus den Werten der Wiederholungen berechnet, so dass ein Schätzwert für den Standardfehler der genannten Durchschnittswerte angegeben werden kann.

Eine Bereinigung der Daten erfolgt durch die Eingabe in das Datenprogramm Visual dBASE, Version 4 (BORLAND INTERNATIONAL INC., 1995). Die Aufbereitung des Datenmaterials sowie die deskriptiven statistischen Analysen erfolgte mit Hilfe des Programmpaketes Statistic Analysis System Version 8.1 (SAS INSTITUTE INC., 2000) mit dem die Mittelwerte und Standardabweichungen berechnet wurden. 


\section{Ergebnisse}

Anhand des im Rahmen dieser Arbeit entwickelten Simulationsprogramms war es möglich, Informationen über die Veränderungen genetischer Variablen der Merkmale Dressur, Springen und Osteochondrose bei Durchführung von Selektionsmaßnahmen mit dem Ziel der Reduzierung der Osteochondrose-Frequenz zu gewinnen. Im Folgenden soll ein Überblick gegeben werden über die Ergebnisse bei Anwendung der verschiedenen genetischen Modelle, Selektionsmethoden und intensitäten. Die phänotypische Ausgangsfrequenz der Osteochondrose beträgt bei allen Modellen und Selektionsmethoden 0,2 in der Basispopulation. Um die Unterschiede zwischen den erzielten Ergebnissen besser bewerten und einordnen zu können, werden die mittleren Standardfehler der genetischen Modelle bei Anwendung der jeweiligen Selektionsgewichte- und Methoden angegeben.

\subsection{Reduktion der OC-Frequenz bei Anwendung der genetischen Modelle I und II innerhalb der einstufigen Selektion}

Die Ergebnisse bei Anwendung des genetischen Modells I (OC als Genort mit zwei Allelen angenommen) und des Modells II (OC ist ein normalverteiltes Merkmal) innerhalb der einstufigen Selektionsmethode sind in der folgenden Tabelle 8 gegenübergestellt.

Tabelle 8: OC-Frequenzen bei Anwendung der genetischen Modelle I und II innerhalb der einstufigen Selektionsmethode

\begin{tabular}{|c|c|c|c|c|}
\hline \multicolumn{3}{|c|}{ Selektionsgewichte auf } & \multicolumn{2}{|c|}{ OC-Frequenz $^{1)}$} \\
\hline Dressur & Springen & OC & Modell I ${ }^{2)}$ & Modell II ${ }^{3)}$ \\
\hline 0,4 & 0,4 & 0,0 & 0,184 & 0,205 \\
\hline 0,4 & 0,4 & 0,2 & 0,087 & 0,171 \\
\hline 0,4 & 0,4 & 0,4 & 0,056 & 0,148 \\
\hline 0,4 & 0,4 & 0,8 & 0,040 & 0,139 \\
\hline
\end{tabular}


Die Anwendung des genetischen Modells I führt bei einer Gewichtung von 0,4/0,4/0,2 der Merkmale zu einer OC-Frequenz von 0,087 in der fünften Generation. Das genetische Modell II führt zu einer OC-Frequenz von 0,171 in der fünften Generation bei Anwendung der gleichen Selektionsgewichte. Beim Vergleich der OC Reduktion durch die genetischen Modelle I und II innerhalb der einstufigen Selektionsmethode, bei der die gesamte Population (Hengste und Stuten) einer röntgenologischen Untersuchung unterzogen wird, zeigt sich somit, dass das genetische Modell I zu einer deutlicheren Reduktion der OC-Frequenz führt.

Hinsichtlich der Anwendung unterschiedlicher Gewichtungen der Merkmale sinkt die OC-Frequenz erwartungsgemäß am meisten bei der verstärkten Gewichtung der Osteochondrose $(0,4 / 0,4 / 0,8)$. Die Gewichtung 0,4/0,4/0,2 wird jedoch als die optimalste Gewichtung angesehen, die auch am realistischsten die Gewichtung der Merkmale in der Praxis widerspiegelt. Aus diesem Grund werden auch die Ergebnisse bei Anwendung dieser Gewichte für den Vergleich der beiden Modelle herangezogen.

Die folgende Abbildung 4 stellt den unterschiedlichen Selektionserfolg der beiden Modelle bei Anwendung dieser Gewichtung grafisch dar.

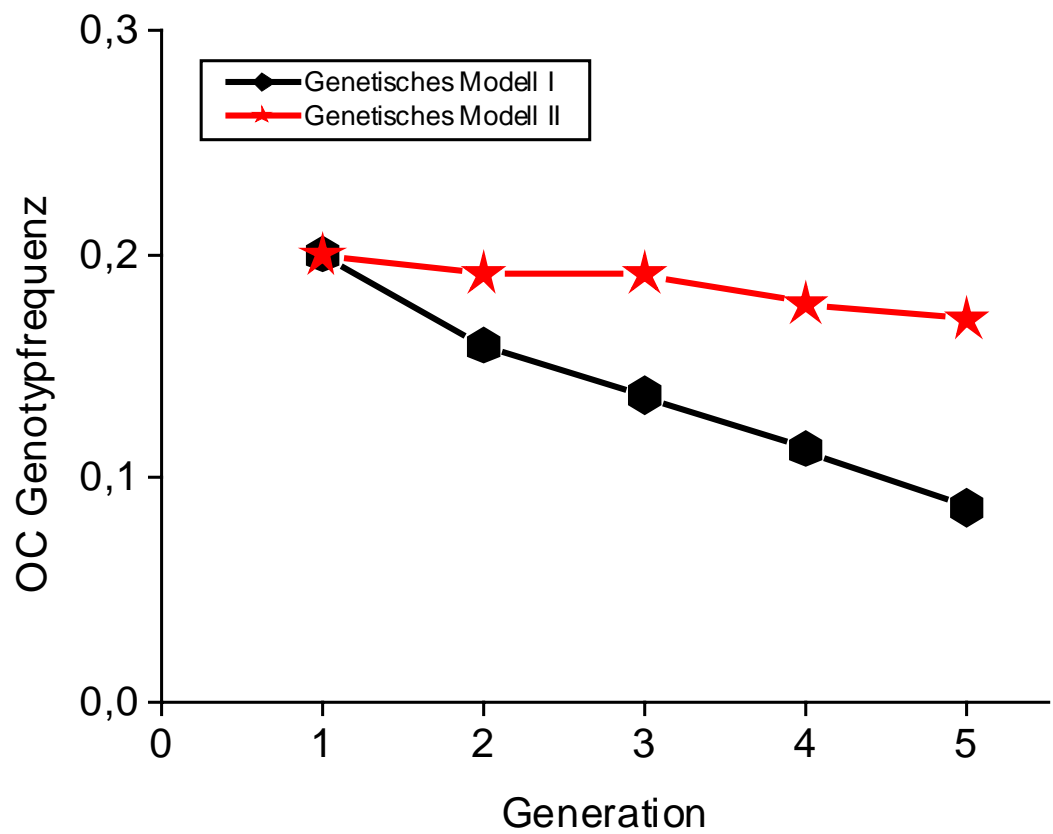

Abbildung 4: Veränderung der OC-Frequenzen über fünf Generationen (Selektionsgewichte 0,4/0,4/0,2) 


\subsection{Reduktion der OC-Frequenz bei Anwendung der genetischen Modelle I und II innerhalb der zweistufigen Selektion}

Im Hinblick darauf, die reale Situation in der Pferdezucht simulieren zu wollen, wurde das einstufige Selektionsmodell zum zweistufigen Modell weiterentwickelt, das dem Selektionsschema bei der Hengstkörung nachempfunden ist. Die Anwendung der beiden genetischen Modelle führte zu folgenden Ergebnissen, die in Tabelle 9 dargestellt sind.

Tabelle 9: OC-Frequenzen bei Anwendung der genetischen Modelle I und II innerhalb der zweistufigen Selektionsmethode

\begin{tabular}{|ccc|cc|}
\hline \multicolumn{2}{|c|}{ Selektionsgewichte auf ${ }^{\mathbf{1}}$} & \multicolumn{2}{c|}{ OC-Frequenz $^{2)}$} \\
Dressur & Springen & OC & Modell I $^{\text {3) }}$ & Modell II $^{\text {4) }}$ \\
\hline 0,4 & 0,4 & 0,0 & 0,205 & 0,205 \\
\hline 0,4 & 0,4 & 0,2 & 0,120 & 0,183 \\
\hline 0,4 & 0,4 & 0,4 & 0,077 & 0,155 \\
\hline 0,4 & 0,4 & 0,8 & 0,059 & 0,145 \\
\hline
\end{tabular}

Auch innerhalb der zweistufigen Selektionsmethode zeigen die Ergebnisse mit 0,120 des genetischen Modells I in der fünften Generation im Gegensatz zu 0,183 des genetischen Modells II bei Anwendung der Gewichtungen, die den Heritabilitäten der Merkmale entsprechen, dass das genetische Modell I im Gegensatz zum Modell II zu einer deutlicheren Reduktion der OC-Frequenz führt.

\subsection{Reduktion der OC-Frequenz bei Anwendung der ein- oder zweistufigen Selektionsmethode}

Die Selektion einer Population gegen Osteochondrose im Rahmen einer ein- oder zweistufigen Selektion bedarf unterschiedlicher organisatorischer und finanzieller Aufwendungen. 
In diesem Zusammenhang ist besonders interessant, welche Fortschritte bei der Reduzierung der OC-Frequenz mit den unterschiedlichen Selektionsmethoden erzielt werden können.

Beim Vergleich der ein- und zweistufigen Selektionsmethode wird deutlich, dass die einstufige Selektion bei Anwendung der beiden genetischen Modelle und verschiedener Selektionsszenarien zu einer deutlich stärkeren Reduktion der OCFrequenz führt. Dies ist darauf zurückzuführen, dass bei der einstufigen Selektionsmethode eine röntgenologische Überprüfung der gesamten Population (Hengste+ Stuten) durchgeführt wird. Die zweistufige Selektion bezieht hingegen lediglich die Hengste mit einer überragenden Leistung in Dressur und Springen in röntgenologische Untersuchungen ein. Aufgrund der erheblichen finanziellen Aufwendungen, die für die Durchführung der einstufigen Selektion aufgebracht werden müssten, um eine röntgenologische Untersuchung der gesamten Population durchzuführen, wird die praktische Umsetzung dieses Selektionsmodells als schwierig erachtet. Beim zweistufigen Selektionsmodell findet eine bessere Orientierung an Praxisbedingungen aus der Pferdezucht statt, so dass dieses Modell als Basismodell für weitere Entwicklungen genutzt wurde.

\subsection{Veränderungen der Zuchtwerte der Leistungsmerkmale bei unterschiedlicher Gewichtung von OC}

Von besonderer Bedeutung bei der Reduzierung der OC-Frequenz ist die Frage, ob sich durch Anwendung verschiedener Gewichtungen gegen Osteochondrose auch die Zuchtwerte für Dressur und Springen verändern.

$\mathrm{Zu}$ diesem Zweck wurden die Zuchtwerte bei Anwendung verschiedener Selektionsgewichte gegen OC bei der einstufigen Selektion unter Anwendung des genetischen Modells I in Tabelle 10 dargestellt. 
Tabelle 10: Veränderungen der Zuchtwerte für Dressur und Springen bei Anwendung unterschiedlicher Selektionsgewichte gegen OC (einstufige Selektion, genetisches Modell I)

\begin{tabular}{|lcc|cc|}
\hline \multicolumn{2}{|c|}{ Selektionsgewichte auf } & \multicolumn{2}{c|}{ Mittelwerte der Zuchtwerte } \\
Dressur & Springen & OC & Dressur ${ }^{\text {1) }}$ & Springen 2) \\
\hline 0,4 & 0,4 & 0,0 & 1,738 & 1,780 \\
\hline 0,4 & 0,4 & 0,2 & 1,709 & 1,748 \\
\hline 0,4 & 0,4 & 0,4 & 1,698 & 1,701 \\
\hline 0,4 & 0,4 & 0,8 & 1,642 & 1,678 \\
\hline 1) mittlerer Standardfehler $=0,003$ & 2) mittlerer Standardfehler $=0,003$ & \\
\hline
\end{tabular}

Analog zu den veränderten Selektionsgewichten auf $\mathrm{OC}$ verändern sich die Zuchtwerte für die Merkmale Dressur und Springen. Je höher das Selektionsgewicht für OC gewählt wird, desto höher ist der Fortschritt bei der Reduzierung der OCFrequenz; umso geringer sind aber auch die Fortschritte bei den Zuchtwerten der Leistungsmerkmale Dressur und Springen. Abbildung 5 verdeutlicht diese Zusammenhänge:

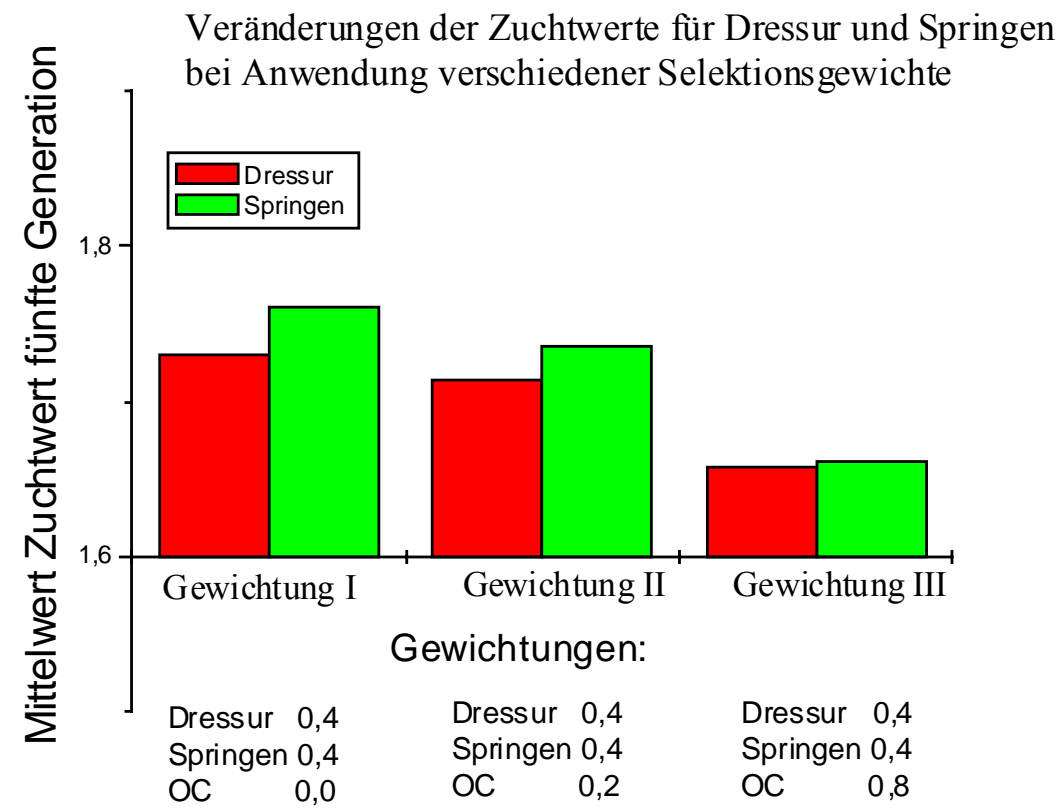

Abbildung 5: Veränderungen der Zuchtwerte für Dressur und Springen bei Anwendung verschiedener Selektionsgewichte 
Die Bewertung der bei der einstufigen Selektion erzielten Ergebnisse führte zu der Erkenntnis, dass mit der Gewichtung 0,4/0,4/0,2 für die einzelnen Merkmale eine optimale Gewichtung erreicht wurde, die auch der in der Praxis durchgeführten Gewichtung entspricht.

\subsection{Einfluss der Remontierungsraten auf die Reduktion der OC-Frequenz und die Zuchtwerte der Leistungsmerkmale}

Das zweistufige Selektionsmodell ermöglicht es, Remontierungsraten auf der ersten Stufe anzugeben, die maßgebend dafür sind, wie streng auf dieser Stufe in Dressur und Springen selektiert wird. Aufgrund der Angabe der Remontierungsrate auf der ersten Stufe und der fixen Gesamtremontierungsrate berechnet sich dann innerhalb des Programms die Remontierungsrate auf der zweiten Stufe, bei der ebenfalls eine Selektion auf Dressur und Springen erfolgt, zusätzlich wird der OC Status in der Selektion berücksichtigt.

Die Anwendung verschiedener Remontierungsraten bei dem genetischen Modell II zeigt in Tabelle 11 folgende Ergebnisse:

Tabelle 11: Mittelwerte der OC-Frequenzen und der Zuchtwerte der Leistungsmerkmale bei Anwendung der zweistufigen Selektion mit unterschiedlichen Remontierungsraten (genetisches Modell II mit zwei Klassen)

\begin{tabular}{|c|c|c|c|c|}
\hline \multicolumn{2}{|c|}{ Remontierungsrate $^{1)}$} & \\
\hline Erste Stufe & Zweite Stufe & Frequenz $^{\text {2) 3) }}$ & Dressur ${ }^{4)}$ & Springen ${ }^{5)}$ \\
\hline 0,01 & 1 & 0,205 & 1,763 & 1,770 \\
\hline 0,02 & 0,5 & 0,194 & 1,721 & 1,766 \\
\hline 0,05 & 0,2 & 0,183 & 1,662 & 1,745 \\
\hline 0,1 & 0,1 & 0,183 & 1,662 & 1,745 \\
\hline
\end{tabular}

2) Durchschnittsfrequenz für OC in der ersten Generation=0,2 3) Mittlerer Standardfehler $=0,001$

4) Mittlerer Standardfehler $=0,004$

5) Mittlerer Standardfehler $=0,004$

Anhand der Ergebnisse zeigt sich, dass eine Remontierungsrate von 0,05 auf der ersten Selektionsstufe und von 0,2 auf der zweiten Stufe unter Annahme gleicher ökonomischer Gewichtung der Selektionsmerkmale (vergleiche dazu Tabelle 34) zu der stärksten Reduktion der OC-Frequenz führt. 
Eine Erhöhung der Remontierungsrate auf der ersten und eine niedrigere Remontierungsrate auf der zweiten Stufe führen $\mathrm{zu}$ keinem weiteren Selektionserfolg. Die Ergebnisse, die bei Anwendung der Remontierungsraten 0,05 auf der ersten und 0,2 auf der zweiten Selektionsstufe berechnet wurden, werden deshalb auch zum Vergleich der Selektionserfolge der verschiedenen Modelle verwendet.

Ebenso wie eine unterschiedliche Gewichtung der Merkmale bei der Selektion gegen OC, können auch verschiedene Remontierungsraten Einfluss auf die Zuchtwerte der Leistungsmerkmale Dressur und Springen nehmen. Die größten Fortschritte werden bei den Remontierungsraten 0,01 auf der ersten und 1 auf der zweiten Selektionsstufe erreicht, da hier die schärfste Selektion für Dressur und Springen angewandt wird, und keine Selektion für OC erfolgt. Diese Fortschritte werden mit abnehmender Selektionsschärfe für Dressur und Springen und mit zunehmender Selektionsschärfe für OC innerhalb der nächsten angewandten Remontierungsraten geringer, bis bei der Anwendung der Remontierungsraten 0,05 auf der ersten und 0,2 auf der zweiten Selektionsstufe die geringsten Fortschritte in den Zuchtwerten für Dressur und Springen erreicht werden.

\subsection{Simulation verschiedener Selektionsszenarien gegen OC aus der Pferdezuchtpraxis/ Hengstkörung}

Auf der Basis des zweistufigen genetischen Modells II wurden verschiedene Selektionsmethoden entwickelt, die in der Praxis angewandte Auswahlverfahren bei der Hengstkörung simulieren sollen. Diese Selektionsszenarien verlaufen wie das genetische Modell mit zwei Klassen auf zwei Selektionsstufen, auf der zweiten Stufe findet die Selektion gegen OC Berücksichtigung.

\section{A) Phänotypische Darstellung der OC in vier Klassen}

Beim Modell mit vier phänotypischen Klassen erfolgt eine Einteilung des Phänotyps in vier Röntgenklassen, die eine Einordnung des röntgenologischen Status eines Hengstes ermöglichen. Bei der Körung kann dieser Status durch überdurchschnittliche Leistungen in Dressur und Springen ausgeglichen werden. 


\section{B) Ausschlussverfahren}

Die Weiterentwicklung des Modells mit vier Klassen simuliert einen Ausschluss der Hengste bei der Körung, die aufgrund ihrer röntgenologischen Befunde in die Röntgenklassen drei und/oder vier eingeteilt wurden. Diese beiden Selektionsszenarien mit und ohne Ausschluss werden in der Praxis bei Hengstkörungen angewendet und können hier direkt verglichen werden.

\section{C) Selektion der Hengste nach Zuchtwertschätzergebnissen in OC und Dressur/Springen}

Es wurde eine Zuchtwertschätzung für die Osteochondrose entwickelt, die ein frühes Vorliegen von Informationen anhand von Eigen- und Halbgeschwisterleistung ermöglichen würde.

Im Folgenden werden die Auswirkungen dieser verschiedenen Selektionsmethoden auf die OC-Frequenz und die Zuchtwerte der Leistungsmerkmale dargestellt und die Selektionsvarianten hinsichtlich ihrer Effizienz verglichen.

\subsection{1.a. Modell mit vier Klassen}

Um den Fortschritt durch die Weiterentwicklung des genetischen Modells II mit zwei Klassen zum Modell mit vier Klassen besser beurteilen zu können, werden die Ergebnisse hinsichtlich der Reduktion der OC-Frequenz bei Anwendung verschiedener Remontierungsraten in der folgenden Tabelle 12 gegenübergestellt:

Tabelle 12: OC-Frequenzen bei Darstellung der OC in zwei bzw. vier phänotypischen Klassen

\begin{tabular}{|cc|cc|}
\hline \multicolumn{2}{|c|}{ Remontierungsrate $^{\mathbf{1 )}}$} & \multicolumn{2}{c|}{ OC-Frequenz $^{\text {2) }}$} \\
Erste Stufe & Zweite Stufe $^{\text {4-Klassen }}{ }^{\text {3) }}$ & 2-Klassen $^{\text {4) }}$ \\
\hline 0,01 & 1 & 0,205 & 0,205 \\
\hline 0,02 & 0,5 & 0,145 & 0,194 \\
\hline 0,05 & 0,2 & 0,138 & 0,183 \\
\hline 0,1 & 0,1 & 0,138 & 0,183 \\
\hline
\end{tabular}

3) Mittlerer Standardfehler $=0,001$ 4) Mittlerer Standardfehler $=0,001$ 
Der Vergleich der Ergebnisse zwischen den beiden Modellen zeigt, dass durch die Einteilung des Phänotyps in vier Klassen eine stärkere Reduktion der OC-Frequenz in der fünften Generation erreicht werden konnte ( 0,138 bei genetischem Modell II mit vier Klassen vs. 0,183 bei genetischem Modell II mit zwei Klassen).

\subsection{1.b Ausschlussselektion}

Das erste Ausschlussmodell sieht einen Ausschluss aller Hengste, die aufgrund ihres röntgenologischen Status in die Röntgenklasse vier eingeteilt wurden, von weiteren Selektionsentscheidungen vor. Bei der zweiten Ausschlussmethode wird strenger selektiert, alle Hengste mit der Röntgenklasse drei und vier werden auf der zweiten Stufe von der weiteren Selektion ausgeschlossen. Um die Auswirkungen und Erfolge bei der Reduktion der Osteochondrose Frequenz durch die beiden Modelle zu vergleichen, sind die Ergebnisse der beiden Ausschlussselektionsszenarien in Tabelle 13 gegenübergestellt.

Tabelle 13: OC-Frequenzen bei Anwendung der Ausschlussselektionsmodelle

\begin{tabular}{|c|c|c|c|}
\hline \multicolumn{2}{|c|}{ Remontierungsrate $^{1)}$} & \multicolumn{2}{|c|}{ OC-Frequenz $^{2)}$} \\
\hline \multirow[b]{3}{*}{ Erste Stufe } & \multirow[b]{3}{*}{ Zweite Stufe } & Ausschluss & Ausschluss \\
\hline & & Röntgenklasse & Röntgenklasse \\
\hline & & $\mathbf{I I I}+\mathbf{I V}^{3)}$ & IV $^{4)}$ \\
\hline 0,01 & 1 & 0,205 & 0,205 \\
\hline 0,02 & 0,5 & 0,160 & 0,174 \\
\hline 0,05 & 0,2 & 0,142 & 0,160 \\
\hline 0,1 & 0,1 & 0,142 & 0,160 \\
\hline
\end{tabular}

2) Durchschnittsfrequenz für OC in der ersten Generation $=0,2$ 3) Mittlerer Standardfehler $=0,001$

4) Mittlerer Standardfehler $=0,002$

Das Selektionsmodell mit Ausschluss der Hengste, welche die Röntgenklassen drei oder vier aufweisen, führt $\mathrm{zu}$ einer OC-Frequenz von 0,142 in der fünften Generation. Im Vergleich dazu ist bei der Anwendung des Modells, bei dem nur die Hengste mit Röntgenklasse vier ausgeschlossen werden, unter Anwendung der gleichen Selektionsbedingungen eine OC- Frequenz von 0,160 in der fünften Generation zu verzeichnen. 
Erwartungsgemäß führt also das Modell mit Ausschluss der Hengste mit Röntgenklasse drei und vier zu einem verstärkten Selektionserfolg hinsichtlich der Reduzierung der OC-Frequenz.

Der Ausschluss eines Hengstes aus der Zucht aufgrund seines röntgenologischen Status kann bedeuten, dass wertvolle Eigenschaften eines potentiellen Vatertieres in Bezug auf die Leistungsmerkmale Dressur und Springen verloren gehen. Von besonders großem züchterischem Interesse ist deshalb in diesem Zusammenhang die Beobachtung der Veränderungen der Zuchtwerte der Leistungsmerkmale bei Anwendung der verschiedenen Ausschluss-Selektionsmethoden. Tabelle 14 stellt diese Veränderungen bei Anwendung unterschiedlicher Remontierungsraten dar:

Tabelle 14: Mittlere Zuchtwerte der Leistungsmerkmale bei Anwendung der Ausschlussselektionsmodelle

\begin{tabular}{|c|c|c|c|c|c|}
\hline Rem & gsrate $^{1)}$ & Mit & telwerte des & Zuchtwert & e bei: \\
\hline & & $\begin{array}{l}\text { Ausschlus } \\
\text { Röntgenk } \\
\text { III+IV }\end{array}$ & lasse & \begin{tabular}{|r} 
Auss \\
Röntgen
\end{tabular} & $\begin{array}{l}\text { chluss } \\
\text { klasse } \\
\text { IV }\end{array}$ \\
\hline Erste Stufe & Zweite Stufe & Dressur ${ }^{2)}$ & Springen $^{3)}$ & Dressur ${ }^{4)}$ & Springen ${ }^{5)}$ \\
\hline 0,01 & 1 & 1,658 & 1,706 & 1,682 & 1,715 \\
\hline 0,02 & 0,5 & 1,641 & 1,692 & 1,663 & 1,700 \\
\hline 0,05 & 0,2 & 1,632 & 1,671 & 1,649 & 1,689 \\
\hline 0,1 & 0,1 & 1,632 & 1,671 & 1,649 & 1,689 \\
\hline $\begin{array}{l}\text { 1) angewendete } \mathrm{Se} \\
\text { 3) mittlerer Standat }\end{array}$ & $\begin{array}{l}\text { chte: Dressur 0,4, Spr } \\
04\end{array}$ & $0,4, \mathrm{OC} 0,2$ & 2) mittlerer Sta & Iddardfehler $=0,0$ & \\
\hline 3) mittlerer Standal & & & 4) mittlerer Sta & & \\
\hline
\end{tabular}

Bei der Beobachtung der Veränderungen der Zuchtwerte für Dressur und Springen dreht sich die vorherige Rangfolge um, denn hier macht das Modell, bei dem nur ein Ausschluss der Röntgenklasse vier erfolgt, im Vergleich zu dem Ausschlussmodell, bei dem strenger selektiert wird, bessere Fortschritte in den Zuchtwerten der Leistungsmerkmale Dressur und Springen. 
4.6.1.1. Vergleich des Modells mit vier Klassen und der Ausschlussselektion

Die Selektionsmethode mit vier phänotypischen Klassen, bei der durch eine gute Leistung in Dressur und Springen ein röntgenologischer Status der Klasse drei oder vier ausgeglichen werden kann; und die Weiterentwicklung dieses Modells, bei der die Röntgenklassen drei und vier oder nur vier von der Zucht ausgeschlossen werden, stellen angewandte Selektionsmethoden in der Praxis bei der Hengstkörung dar. Um die unterschiedlichen Fortschritte bei der Reduzierung der OC-Frequenz mit Hilfe des Modells ohne Ausschluss, und des Modells mit Ausschluss der röntgenologisch schlecht eingestuften Hengste $\mathrm{zu}$ verdeutlichen, werden die Ergebnisse in den folgenden Tabellen 15/16 gegenübergestellt.

Tabelle 15: OC-Frequenzen bei Anwendung des Modells mit vier Klassen ohne Ausschluss und der Anwendung der Ausschlussselektionen mit Ausschluss der Röntgenklasse IV/Ausschluss der Röntgenklassen III und IV

\begin{tabular}{|c|c|c|c|c|}
\hline \multicolumn{2}{|c|}{ Remontierungsrate $^{1)}$} & \multicolumn{3}{|c|}{ OC-Frequenz $^{2)}$} \\
\hline Erste Stufe & Zweite Stufe & $\begin{array}{l}\text { 4-Klassen } \\
\text { Modell }^{3)}\end{array}$ & $\begin{array}{c}\text { Ausschluss } \\
\text { Röntgenkl. }^{4)} \\
\text { IV }\end{array}$ & $\begin{array}{c}\text { Ausschluss } \\
\text { Röntgenkl. }^{5)} \\
\text { III+IV }\end{array}$ \\
\hline 0,01 & 1 & 0,205 & 0,205 & 0,205 \\
\hline 0,02 & 0,5 & 0,145 & 0,174 & 0,160 \\
\hline 0,05 & 0,2 & 0,138 & 0,160 & 0,142 \\
\hline 0,1 & 0,1 & 0,138 & 0,160 & 0,142 \\
\hline
\end{tabular}

1) angewendete Selektionsgewichte: Dressur 0,4, Springen 0,4, OC 0,2
2) Durchschnittsfrequenz für OC in der ersten Generation $=0,2$
3) mittlerer Standardfehler $=0,001$
4) mittlerer Standardfehler $=0,001$
5) mittlerer Standardfehler $=0,002$

Der Vergleich der Selektionsmethoden mit Ausschluss der Röntgenklasse vier und ohne Ausschluss zeigt, dass die Selektion ohne Ausschluss von Hengsten bei der Körung zu einer deutlich geringeren OC-Frequenz in der Population führt (0,138 vs. 0,160 in der fünften Generation). 
Beim Vergleich der Selektionsmethoden mit Ausschluss der Röntgenklasse drei und vier und der Methode ohne Ausschluss zeigt sich hingegen kein deutlicher Unterschied in der Reduzierung der OC-Frequenz: das Modell ohne Ausschluss führt $\mathrm{zu}$ einer OC-Frequenz von 0,138 in der fünften Generation, das Modell mit Ausschluss reduziert die OC-Frequenz auf einen Wert von 0,142 bei Anwendung der Remontierungsraten 0,05 auf der ersten Selektionsstufe und 0,2 auf der zweiten Selektionsstufe in beiden Modellen. Bei dem Vergleich der beiden Selektionsmethoden mit und ohne Ausschluss ist besonders im Hinblick auf den Zuchtfortschritt in den Leistungsmerkmalen interessant, wie sich der genetische Trend bei der Anwendung der beiden Selektionsmethoden in den Merkmalen Dressur und Springen verändert.

Tabelle 16: Mittlere Zuchtwerte der Leistungsmerkmale bei Anwendung des Modells mit vier Klassen ohne Ausschluss und der Anwendung der Ausschlussselektionen mit Ausschluss der Röntgenklasse IV/Ausschluss der Röntgenklassen III und IV

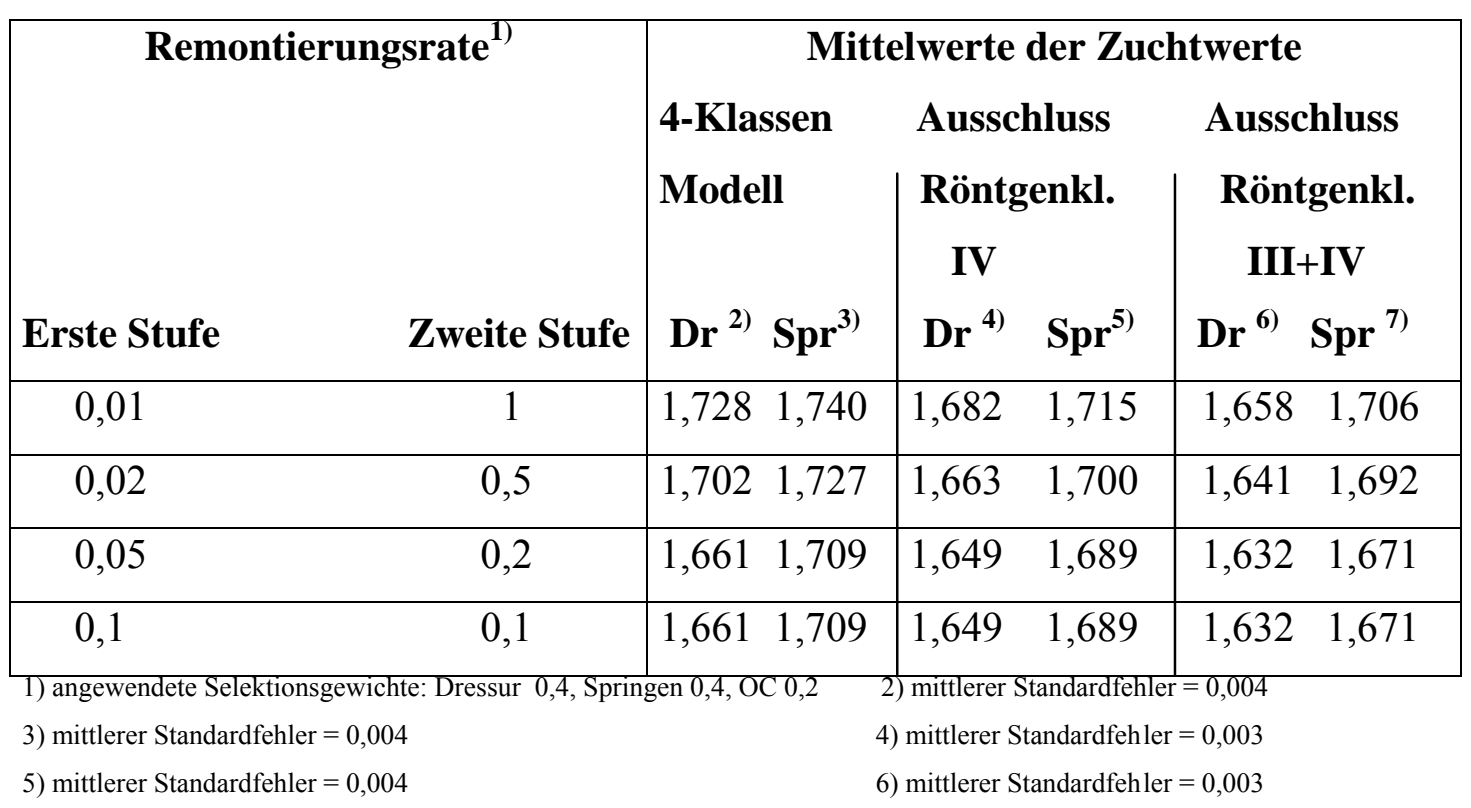

Der Vergleich der Fortschritte in den Zuchtwerten der Leistungsmerkmale zwischen den Selektionsmodellen mit und ohne Ausschluss macht deutlich, dass bei dem Modell ohne Ausschluss ein größerer Fortschritt in den Leistungsmerkmalen gemacht wird, als bei den Modellen, bei denen ein Ausschluss von Hengsten aufgrund ihrer Röntgenklasse bei der Körung erfolgt. 
Das Modell mit vier Klassen ohne Ausschluss von Hengsten bei der Körung führt im Vergleich zu den Ausschlussmodellen sowohl hinsichtlich der Reduzierung der OCFrequenz als auch bei den Fortschritten in den Zuchtwerten der Leistungsmerkmale zu besseren Ergebnissen.

\subsection{1.c. Zuchtwertschätzung für OC}

Ausgehend vom genetischen Modell II mit vier phänotypischen Klassen wurde eine Zuchtwertschätzung für die Erkrankung Osteochondrose in das Simulationsprogramm eingebaut. Die Zuchtwerte werden anhand der Eigen- und der Halbgeschwisterleistung in den Leistungsmerkmalen und OC ermittelt. Die Anzahl Halbgeschwister variiert von Pferd zu Pferd, sie liegt zwischen eins und der maximalen Anzahl Halbgeschwister. Bei den Berechnungen wurde die Anzahl von 5, 20 und 40 Halbgeschwistern (HG) angenommen, da von einer größeren Anzahl Halbgeschwister eines Hengstes innerhalb eines Körjahrganges unter Praxisbedingungen nicht ausgegangen werden kann. Die folgende Tabelle 17 zeigt die Ergebnisse der Selektion mit Zuchtwertschätzung bei Anwendung der verschiedenen Remontierungsraten und unterschiedlicher Halbgeschwisteranzahl.

Tabelle 17: OC-Frequenzen bei Selektion der Hengste nach geschätzten Zuchtwerten

\begin{tabular}{|c|c|c|c|c|}
\hline \multicolumn{2}{|c|}{ Remontierungsrate $^{1) 3)}$} & \multicolumn{3}{|c|}{ OC-Frequenz $^{2)}$} \\
\hline Erste Stufe & Zweite Stufe & $5 \mathbf{H G}^{4)}$ & $20 \mathrm{HG}^{5)}$ & $40 \mathrm{HG}^{6)}$ \\
\hline 0,01 & 1 & 0,205 & 0,205 & 0,205 \\
\hline 0,02 & 0,5 & 0,145 & 0,139 & 0,131 \\
\hline 0,05 & 0,2 & 0,130 & 0,124 & 0,121 \\
\hline 0,1 & 0,1 & 0,130 & 0,124 & 0,121 \\
\hline \multicolumn{5}{|c|}{ 1) angewendete Selektionsgewichte: Dressur 0,4 , Springen $0,4, \mathrm{OC} 0,2$} \\
\hline \multicolumn{5}{|c|}{ 2) Durchschnittsfrequenz für $\mathrm{OC}$ in der ersten Generation $=0,2$} \\
\hline \multicolumn{3}{|c|}{ 3) Zuchtwertschätzung anhand der Eigenleistung und n Halbgeschwistern (HG) } & \multicolumn{2}{|c|}{ 4) mittlerer Standardfehler $=0,001$} \\
\hline \multicolumn{3}{|c|}{ 5) mittlerer Standardfehler $=0,002$} & \multicolumn{2}{|c|}{ 6) mittlerer Standardfehler $=0,002$} \\
\hline
\end{tabular}

Die Ergebnisse zeigen, dass mit einer steigenden Anzahl Halbgeschwister eine höhere Reduzierung der OC-Frequenz erreicht werden kann: 0,130 bei 5 HG vs. 0,124 bei $20 \mathrm{HG}$ vs. 0,121 bei $40 \mathrm{HG}$ unter Annahme einer Remontierungsrate von 0,05 auf der ersten und 0,2 auf der zweiten Selektionsstufe. 
Bei der Anwendung dieses Zuchtwertschätzmodells sollen ebenfalls die Veränderungen in den Zuchtwerten der Leistungsmerkmale beobachtet werden. Die folgende Tabelle 18 zeigt die Veränderungen bei Anwendung verschiedener Remontierungsraten und Angabe unterschiedlicher Halbgeschwisteranzahl auf.

Tabelle 18: Mittlere Zuchtwerte der Leistungsmerkmale bei Selektion der Hengste nach geschätzten Zuchtwerten

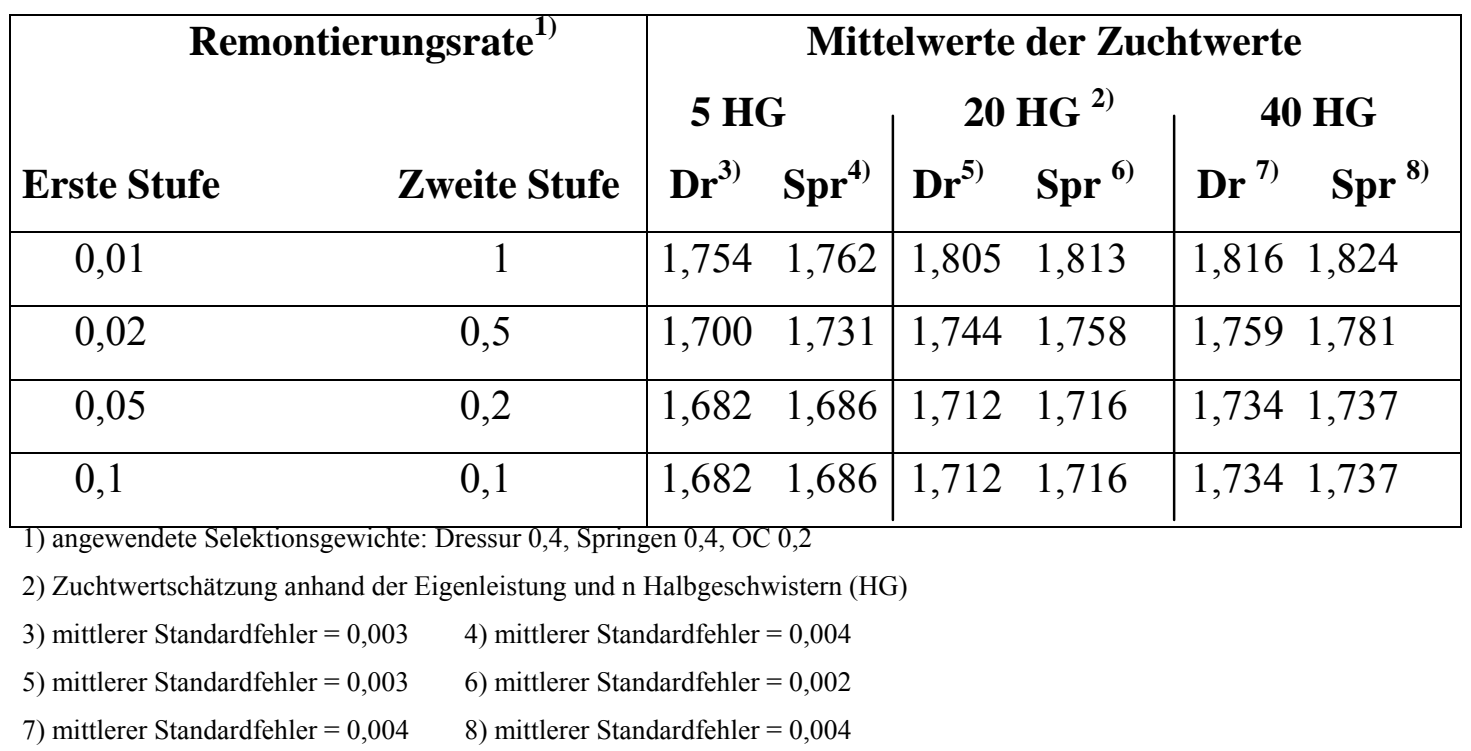

Auch bei den Zuchtwerten für Dressur und Springen werden größere Fortschritte bei einer steigenden Halbgeschwisteranzahl beobachtet.

\subsubsection{Vergleich der Selektionsszenarien /Hengstkörung}

Im Folgenden werden die Modelle, welche die Selektion bei der Körung simulieren, hinsichtlich ihrer Effizienz bei der Reduktion der OC-Frequenz und bezüglich ihrer Auswirkungen auf den genetischen Trend der Leistungsmerkmale in den Tabellen 19/20 verglichen. Bei der Ausschlussselektion wurde das Modell mit Ausschluss der Röntgenklassen drei und vier für den Vergleich gewählt, da dieses Modell auch in der Praxis Anwendung findet. Bei dem Modell mit Zuchtwertschätzung werden die Ergebnisse der Berechnung mit 20 Halbgeschwistern gewählt, da eine Anzahl von 20 Halbgeschwistern realistisch innerhalb eines Körjahrgangs erscheint. 
Tabelle 19: OC-Frequenzen bei Anwendung verschiedener Selektionsverfahren für Hengste

\begin{tabular}{|c|c|c|c|c|}
\hline Rem & ngsrate $^{1)}$ & & C-Frequenz & \\
\hline Erste Stufe & Zweite Stufe & $\begin{array}{l}\text { 4- Klassen } \\
\text { Modell }^{5)}\end{array}$ & $\begin{array}{l}\text { Ausschluss } \\
\text { Modell }^{\text {3) 6) }}\end{array}$ & $\begin{array}{l}\text { ZWS } \\
\text { Modell }^{4)} \text { 7) }\end{array}$ \\
\hline 0,01 & 1 & 0,205 & 0,205 & 0,205 \\
\hline 0,02 & 0,5 & 0,145 & 0,160 & 0,139 \\
\hline 0,05 & 0,2 & 0,138 & 0,142 & 0,124 \\
\hline 0,1 & 0,1 & 0,138 & 0,142 & 0,124 \\
\hline
\end{tabular}

\footnotetext{
2) Durchschnittsfrequenz für OC in der ersten Generation=0,2 $\quad 3$ ) Röntgenklassen III und IV ausselektiert

4) ZWS anhand von $20 \mathrm{HG}$

5) mittlerer Standardfehler $=0,001$

6) mittlerer Standardfehler $=0,002$

7) mittlerer Standardfehler $=0,002$
}

Hinsichtlich der Effizienz bei der Reduzierung der Osteochondrose Frequenz schneidet das Zuchtwertschätzmodell mit einem Wert von 0,124 in der fünften Generation am besten ab. Bei gleichen Remontierungsraten führt das Modell mit vier phänotypischen Klassen zu einem Ergebnis von 0,138 in der fünften Generation. Das Ausschlussmodell führt mit einem Wert von 0,142 zur geringsten Reduzierung der OC-Frequenz bei Anwendung der gleichen Remontierungsraten.

Tabelle 20: Mittlere Zuchtwerte der Leistungsmerkmale bei Anwendung verschiedener Selektionsverfahren für Hengste

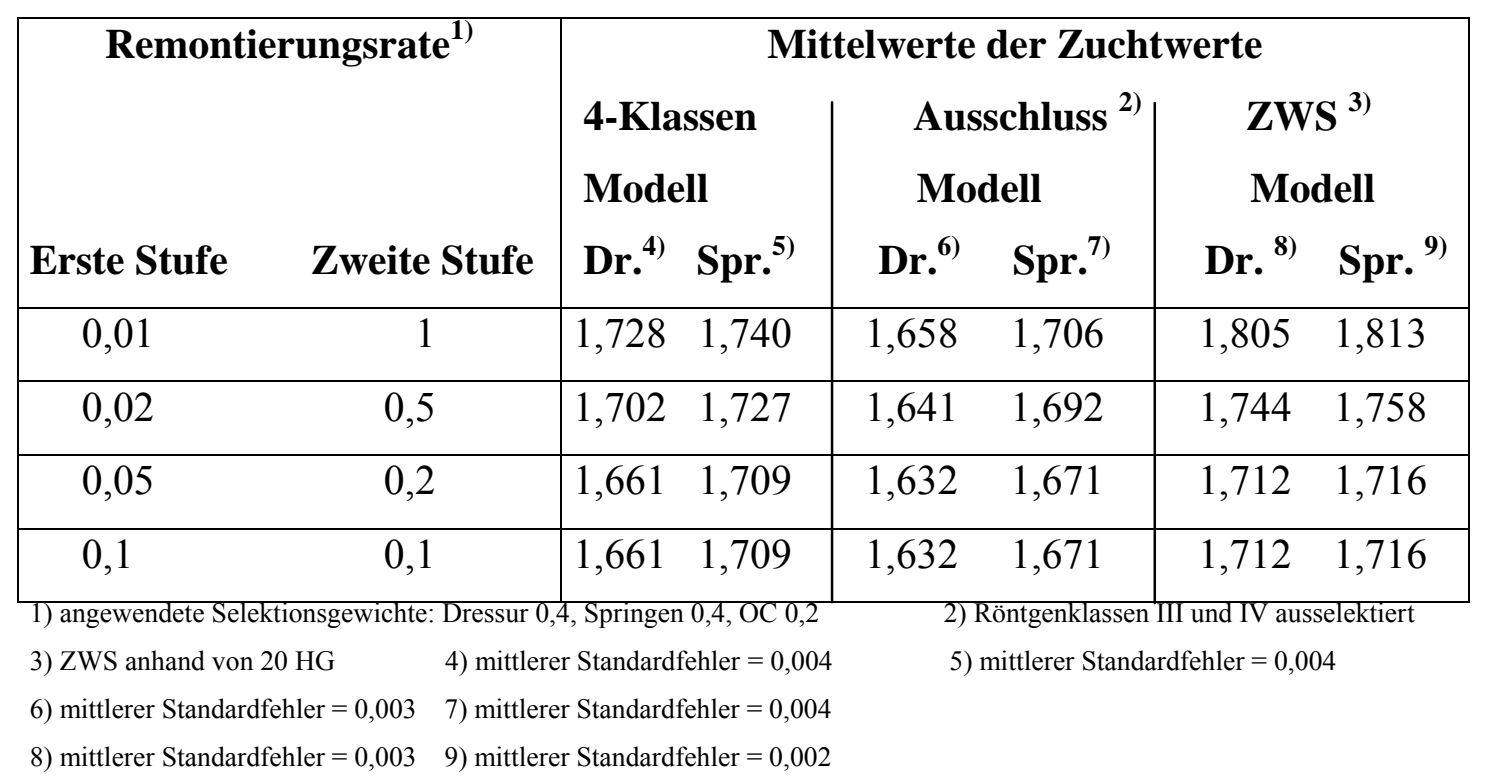


Auch bei der Bewertung der Veränderungen der Zuchtwerte für Dressur und Springen bleibt diese Rangfolge bestehen: das Modell mit Zuchtwertschätzung führt zu den größten Fortschritten bei den Leistungsmerkmalen, gefolgt vom Modell mit vier Klassen, das Modell mit Ausschluss von Röntgenklassen führt zu den geringsten Fortschritten. Zusammenfassend kann zu den Ergebnissen der Selektionsszenarien der Hengstkörung festgestellt werden, dass das Modell mit Zuchtwertschätzung sowohl bei der Reduzierung der OC-Frequenz als auch bei den Zuchtwerten der Leistungsmerkmale Dressur und Springen zu den größten Fortschritten führt.

\subsubsection{Simulation weiterer Selektionsszenarien gegen OC}

\subsubsection{Vorhandensein eines Gentests für $\mathrm{OC}$}

Das Vorhandensein eines Gentests würde in der Zukunft die Diagnose der Osteochondrose auch auf genotypischer Ebene ermöglichen. Dieses Szenario wurde simuliert, indem die Gewichtung des Merkmals OC auf der Genotypseite erfolgt. Um eine möglichst praxisorientierte Anwendung des OC-Gentests zu simulieren, wurde der Gentest innerhalb des zweistufigen Selektionsmodells angewendet, das heißt, dass nur die Pferde, die eine entsprechend gute Leistung in Dressur und Springen zeigten, auf der zweiten Selektionsstufe einem OC Gentest unterzogen würden. Um die unterschiedlichen Ergebnisse bei der Reduzierung der OC-Frequenz zu verdeutlichen, wurden die Ergebnisse der vielleicht in Zukunft möglichen Selektion mit Hilfe des OC- Gentests und der derzeit praktizierten Selektionsmethode mit Hilfe des Röntgenbilds in Tabelle 21 gegenübergestellt.

Tabelle 21: OC-Frequenzen bei Anwendung/Nichtanwendung eines OC-Gentests

\begin{tabular}{|cc|cc|}
\hline \multirow{2}{*}{ Erste Stufe } & Zweite Stufe & Modell $^{\text {3) }}$ & $\begin{array}{c}\text { OC-Frequenz } \\
\text { 4- Klassen Modell } \\
\text { mit OC-Gentest }\end{array}$ \\
\hline 0,01 & 1 & 0,205 & 0,205 \\
\hline 0,02 & 0,5 & 0,145 & 0,124 \\
\hline 0,05 & 0,2 & 0,138 & 0,106 \\
\hline 0,1 & 0,1 & 0,138 & 0,106 \\
\hline
\end{tabular}


Die Ergebnisse zeigen, dass durch die Anwendung eines Gentests für $\mathrm{OC}$ im Vergleich zur Diagnose anhand eines Röntgenbilds eine deutliche Senkung der OCFrequenz erreicht werden könnte.

\subsubsection{Zusätzliche Einbeziehung der Stuten in die Selektion gegen OC}

Bei den bisher vorgestellten zweistufigen Selektionsszenarien fand keine besondere Einbeziehung der Stuten in die Selektion gegen Osteochondrose statt. Um den zusätzlichen Gewinn in der Senkung der OC-Frequenz bei einer Selektion der Stuten und der Hengste zu verdeutlichen, werden die Ergebnisse einer einstufigen Selektion gegen Osteochondrose, in die Stuten und Hengste einbezogen sind, und Ergebnisse einer Selektion, bei der nur Hengste gegen Osteochondrose selektiert wurden; in Tabelle 22 verglichen.

Tabelle 22: OC-Frequenzen bei Selektion Hengste+Stuten vs. Selektion nur der Hengste (genetisches Model I, einstufige Selektion)

\begin{tabular}{|c|c|c|c|c|}
\hline \multicolumn{3}{|c|}{ Selektionsgewichte auf } & \multicolumn{2}{|c|}{ OC-Frequenz ${ }^{1)}$} \\
\hline Dressur & Springen & OC & Stuten+Hengste $^{2)}$ & nur Hengste $^{3 \text { ) }}$ \\
\hline 0,4 & 0,4 & 0,2 & 0,087 & 0,132 \\
\hline 0,4 & 0,4 & 0,4 & 0,056 & 0,078 \\
\hline 0,4 & 0,4 & 0,8 & 0,040 & 0,070 \\
\hline
\end{tabular}

Anhand der Ergebnisse wird deutlich, dass durch die Einbeziehung der Stuten in die Selektion gegen OC ein zusätzlicher Selektionserfolg zu verzeichnen ist. Über die Anwendung verschiedener Selektionsgewichte gegen OC können deutliche Unterschiede bei der Reduktion der OC-Frequenz zwischen der Selektion nur der Hengste und der Selektion der Hengste und Stuten festgestellt werden. 


\section{Diskussion}

Die veränderten Nutzungsansprüche an das Pferd und seine Wandlung vom Arbeitszum Sportpferd haben auch ihre Berücksichtigung in der Zuchtzieldefinition gefunden. Im Rahmen der deutschen Pferdezucht wird ein Zuchtziel verfolgt, welches vor allem eine stetige Verbesserung der leistungsbestimmenden Kriterien vorsieht. Die Züchterschaft bemüht sich erfolgreich, diese Vorgabe in konsequenter Form zu berücksichtigen, was sich auch an den bedeutenden Erfolgen der deutschen Pferdezucht im internationalen Sport ablesen lässt, die in den letzten Jahrzehnten erzielt wurden. Auf der Käuferseite besteht nicht nur ein großes Interesse an hochveranlagten und leistungsbereiten Pferden, aufgrund seiner konditionellen und konstitutionellen Verfassung muss ein Pferd auch den Belastungen des Hochleistungssports wie auch des Freizeitsports standhalten können.

Im Hinblick auf diese Anforderungen und auf mögliche entstehende finanzielle Verluste durch Erkrankungen, notwendige Operationen und die beim Handel des erkrankten Pferdes entstehende Wertminderung scheint eine Integration von funktionalen Merkmalen in züchterische Aktivitäten unumgänglich. Bislang ist jedoch die praktikable und erfolgsversprechende Umsetzung in Zuchtprogramme das primäre Problem.

Im Rahmen der vorliegenden Arbeit wurden verschiedene genetische Modelle und Selektionsmethoden mit Hilfe eines Computersimulationsprogramms entwickelt, um die Auswirkungen von Selektionsmaßnahmen gegen die Gelenkserkrankung Osteochondrose anhand einer simulierten Population nachzuvollziehen. Mit Hilfe der entwickelten genetischen Modelle und Selektionsmaßnahmen wurden verschiedene Selektionsszenarien simuliert, wie diverse Auswahlverfahren bei der Hengstkörung, zusätzliche Einbeziehung der Stuten in die Selektion gegen Osteochondrose sowie das Vorhandensein eines Gentests gegen OC.

Im Vergleich zu anderen Tierarten sind Pferdepopulationen durch ein langes Generationsintervall und eine geringe Nachkommenzahl pro Generation gekennzeichnet. Die Zeitspanne bis Auswirkungen von Selektionsmaßnahmen auf Populationen nachvollzogen werden können, wäre also sehr lang. 
Bei einer solchen Populations- und Vermehrungsstruktur ist der Einsatz von Computersimulationen ein probates Mittel in der Tierzucht. Um mit Hilfe von Simulationsstudien eine möglichst breite und allgemeingültige Aussage zu treffen, sollte die simulierte Population sich in der Struktur und Dynamik den realen Populationen, für die letztendlich die Aussagen gelten sollen, weitestgehend anpassen (BRUNS, 1987). Um dieser Forderung gerecht zu werden, wurde die im Rahmen dieser Untersuchung simulierte Population hinsichtlich Größe, Vermehrungsstruktur, Fruchtbarkeitsparameter und der Angabe der OCAusgangsgenotypfrequenz in Anlehnung an Praxisbedingungen erstellt.

Eine Simulation ist die Nachbildung eines dynamischen Prozesses in einem Modell, um $\mathrm{zu}$ Erkenntnissen zu gelangen, die auf die Wirklichkeit übertragbar sind. SWALVE (1984) bezeichnet die Vorteile einer Simulation folgendermaßen:

1) Die Simulation bietet die Möglichkeit, spezielle Situationen untersuchen zu können, in dem ganz bestimmte Parameter der Simulation variiert werden können

2) Die genetischen Effekte sind von den umweltbedingten Effekten trennbar

3) Das Datenmaterial kann gegenüber praktischen Verhältnissen relativ klein gehalten werden

4) Es muss nicht mit Auszügen aus Datenmaterial gearbeitet werden, die eventuell nicht repräsentativ sind

Die Nachteile der Computersimulation können darin liegen, dass im Gegensatz zu Analysen, die an realen Datensätzen durchgeführt werden, die Übertragbarkeit der Ergebnisse auf reale Populationen nicht garantiert werden kann. Zudem erfordern Computersimulationen einen hohen Aufwand an Rechenzeit und Speicherkapazität, die gelegentlich nicht tragbar sind. 


\subsection{Bewertung der genetischen Modelle}

Da die genaue Vererbung der Osteochondrose bisher noch nicht bestimmt werden konnte, wurden zwei Modelle entwickelt, die eine Annahme treffen über das mögliche genetische Modell der Osteochondrose.

Im genetischen Modell I wird OC als ein Genlocus mit zwei Allelen angenommen ( $a \mathrm{a}=\mathrm{OC}$ befallen, Aa und AA= OC frei). Die homozygot befallenen Pferde mit dem Genotyp aa und die OC freien Pferde mit dem Genotyp AA werden den dementsprechenden Klassen zugeteilt. Die heterozygot befallenen Pferde mit dem Genotyp Aa werden in der Klasse der nicht befallenen Pferde zugeordnet und somit als nicht befallen eingestuft. Der Phänotyp entspricht bei diesem Modell dem definierten Genotyp. Der Erbgang, mit dem sich OC in diesem Modell vererbt, ist autosomal rezessiv. Die Problematik möglicher Fehler bei der Diagnose der Osteochondrose (ungenaue Röntgenbilder, Fehler bei der Interpretation der Befunde) wurde durch die Angabe einer Penetranz des OC- Merkmals in Höhe von 0,8 berücksichtigt. Der Wert 0,8 bedeutet, dass bei der röntgenologischen Untersuchung von z.B.100 OC-befallenen Pferden, die Erkrankung bei 20 Pferden aufgrund der oben genannten Probleme nicht erkannt wird.

Im zweiten genetischen Modell ist die Osteochondrose ein phänotypisches Merkmal mit einer zugrundegelegten Normalverteilung. Anhand der Osteochondrose Frequenz wird eine Festgrenze definiert, die den Phänotyp in zwei Klassen einteilt: OC positiv und OC negativ. Ein Vorteil dieses Modells ist, dass hier Korrelationen zwischen OC und den Leistungsmerkmalen angenommen sowie Umwelteffekte eingebaut werden könnten. 
In der folgenden Tabelle 23 sind die Ergebnisse bei Anwendung der beiden genetischen Modelle innerhalb der einstufigen Selektionsmethode, bei der eine röntgenologische Überprüfung der gesamten Population (Hengste und Stuten) erfolgt, dargestellt.

Tabelle 23: OC-Frequenzen bei Anwendung der genetischen Modelle I und II innerhalb der einstufigen Selektionsmethode

\begin{tabular}{|c|c|c|c|c|}
\hline \multicolumn{3}{|c|}{ Selektionsgewichte auf } & \multicolumn{2}{|c|}{ OC-Frequenz $^{1)}$} \\
\hline Dressur & Springen & OC & Modell I ${ }^{2)}$ & Modell II $^{3)}$ \\
\hline 0,4 & 0,4 & 0,0 & 0,184 & 0,205 \\
\hline 0,4 & 0,4 & 0,2 & 0,087 & 0,171 \\
\hline 0,4 & 0,4 & 0,4 & 0,056 & 0,148 \\
\hline 0,4 & 0,4 & 0,8 & 0,040 & 0,139 \\
\hline
\end{tabular}

Die Ergebnisse zeigen, dass das genetische Modell I zu einer deutlicheren Senkung der Osteochondrose Frequenz führt als das genetische Modell II. Diese Überlegenheit des Modells I gegenüber dem Modell II ist darauf zurückzuführen, dass der Unterschied zwischen dem Genotyp und dem Phänotyp des Merkmals OC in Modell I nur durch die Penetranz definiert wird. Die Selektion gegen OC ist somit weniger beeinflusst durch Umwelteffekte und führt deshalb zu größeren Erfolgen bei der Senkung der OC- Frequenz.

Um eine vollständige Bewertung der beiden genetischen Modelle zu gewährleisten, sollte auch eine Betrachtung der Ergebnisse innerhalb der zweistufigen Selektion erfolgen (Tabelle 24). Hier werden nur die Hengste der simulierten Population in die Selektion gegen OC mit einbezogen, um das Auswahlverfahren bei der Hengstkörung zu simulieren. Die Selektion findet auf zwei Stufen statt: die erste Stufe beinhaltet die Selektion auf Dressur und Springen, auf der zweiten Stufe wird erneut in Dressur und Springen selektiert zusätzlich werden die Hengste, die in diesen Merkmalen überragende Leistungen gezeigt haben, einer röntgenologischen Überprüfung ihres OC-Status unterzogen. 
Tabelle 24: OC-Frequenzen bei Anwendung der genetischen Modell I und II innerhalb der zweistufigen Selektionsmethode

\begin{tabular}{|ccc|cc|}
\hline \multicolumn{3}{|c|}{ Selektionsgewichte auf } & \multicolumn{2}{c|}{ OC-Frequenz $^{\text {1) }}$} \\
Dressur & Springen & OC & Modell I $^{\text {3) }}$ & Modell II $^{\text {) }}$ \\
\hline 0,4 & 0,4 & 0,0 & 0,205 & 0,205 \\
\hline 0,4 & 0,4 & 0,2 & 0,120 & 0,183 \\
\hline 0,4 & 0,4 & 0,4 & 0,077 & 0,155 \\
\hline 0,4 & 0,4 & 0,8 & 0,059 & 0,145 \\
\hline
\end{tabular}

2) Durchschnittsfrequenz für OC in der ersten Generation $=0,2$ 3) mittlerer Standardfehler $=0,002$

4) mittlerer Standardfehler $=0,001$

Die bereits angedeuteten höheren Selektionserfolge bei Reduzierung der OCFrequenz durch das genetische Modell I setzen sich auch bei der Anwendung der zweistufigen Selektionsmethode fort. Das genetische Modell I führt auch hier zu einer stärkeren Reduzierung der OC-Frequenz als das genetische Modell II. Diese unterschiedlichen Fortschritte bei der Reduzierung der OC-Frequenz sind auch hier auf die oben bereits angeführten Gründe und die unterschiedliche Konstruktion der genetischen Modelle zurückzuführen. Das genetische Modell I geht von der Annahme aus, dass die Entstehung und die Ausprägung der Osteochondrose auf ein einziges Gen zurückzuführen ist, der multifaktorielle Hintergrund und die unterschiedliche Ausprägung der Befunde bei befallenen Pferden sprechen jedoch gegen diesen Ansatz. In der Tierzucht gibt es jedoch kaum Merkmale, die nur von einem Genort beeinflusst werden, meist liegt eine Beeinflussung durch mehre Genorte vor.

Anhand dieser Gründe kann davon ausgegangen werden, dass durch das genetische Modell II eine bessere Annährung an das wahre genetische Modell der Osteochondrose erreicht wurde. Hier wird OC als eine biologische Variable beschrieben, die neben genetischen Faktoren von vielen zufälligen Umwelteffekten beeinflusst wird, und somit keinen so großen Selektionserfolg erzielen kann wie das genetische Modell I. Aufgrund dieser Bewertung dient das genetische Modell II als Selektionsmodell, auf dessen Basis die weiteren Selektionsmodelle entwickelt wurden. 


\subsection{Bewertung der Selektionsmethoden}

Um die Anwendung verschiedener Selektionsmethoden zu simulieren, wurden die ein- und die zweistufigen Selektionsmethoden entwickelt und innerhalb der beiden genetischen Modelle angewendet.

Bei der einstufigen Selektion vollzieht sich die Selektion der drei Merkmale Dressur, Springen, OC innerhalb eines Schritts. Bei der zweistufigen Selektion findet auf der ersten Stufe die Selektion für die Merkmale Dressur und Springen statt. Die zweite Selektionsstufe beinhaltet ebenfalls eine Selektion auf Dressur und Springen sowie eine Selektion gegen Osteochondrose. Zusätzlich zur unterschiedlichen Gewichtung der Merkmale Dressur, Springen und OC kann die Selektionsschärfe durch die Angabe von Remontierungsraten auf der ersten Selektionsstufe variiert werden. Je geringer die Remontierungsrate angegeben wird, desto schärfer ist die Selektion.

Beim Vergleich der Reduktion der OC- Frequenz durch die ein- und die zweistufige Selektionsmethode zeigt sich, sich dass die Anwendung der einstufigen Selektion im Gegensatz zur zweistufigen zu einem vermehrten Absinken der OC- Frequenz führt. Der vermehrte Erfolg der einstufigen Selektion ist darauf zurückzuführen, dass hier bei allen Pferden eine röntgenologische Untersuchung auf den OC- Status durchgeführt wird. Auch die Stuten sind hier in die Selektion mit einbezogen worden und tragen zu der deutlichen Reduktion der OC- Frequenz bei. Die zweistufige Selektion soll das Auswahlszenario bei der Körung der Hengste simulieren und vollzieht sich deshalb auf zwei Selektionsstufen, wie sie auch bei der Körung von den Hengsten bewältigt werden muss. Um diese Simulation der Praxisbedingungen auch bei den Ergebnissen fortzuführen, wurden nur die Hengste in die Selektion gegen OC miteinbezogen. Die Stuten werden lediglich in den Merkmalen Dressur und Springen selektiert. Somit sind weniger Pferde in die Selektion einbezogen, was auch zu einem geringeren Selektionserfolg hinsichtlich der Reduzierung der OCFrequenz führt. 
In finanzieller Hinsicht bedeutet die röntgenologische Untersuchung der gesamten Population (Hengste und Stuten), wie sie bei der einstufigen Selektion durchgeführt wird, einen großen Aufwand. Eine röntgenologische Untersuchung zur genauen Lokalisation möglicher OC-Befunde, die die Anfertigung von 18 Röntgenbildern umfasst, beläuft sich laut der Gebührenordnung für Tierärzte (GOT) auf ca. $300 €$.

Dieser finanzielle Aufwand müsste für jedes Pferd der Population aufgebracht werden, die in dieser Studie aus 20.000 Pferden besteht. Die Kosten würden somit bei der Durchführung dieser Selektionsmethode 5.931.200 € betragen. Diese Summe stellt einen erheblichen finanziellen Aufwand dar, zusätzlich stellt sich die Frage, wer diese Kosten tragen soll.

Die zweistufige Selektionsmethode führt in züchterischer Hinsicht zu einem geringeren Erfolg bei der Senkung der OC-Frequenz, ist aber in finanzieller Hinsicht ein Gewinn. Nur die Hengste, die überdurchschnittliche Leistungen in Dressur und Springen zeigen, werden hier einer röntgenologischen Untersuchung auf ihren OCStatus unterzogen. Die Anwendung dieser Methode gegenüber der einstufigen Selektion stellt somit eine deutliche finanzielle Erleichterung dar, da hier bei der Anwendung einer Remontierungsrate von 0,05 auf der ersten Selektionsstufe von 10000 Hengsten der simulierten Population 500 Hengste röntgenologisch untersucht werden, dies würde weitaus geringere Kosten in Höhe von $148.280 €$ nach sich ziehen. Anhand dieser Ergebnisse wird deutlich, dass sich die züchterische Bearbeitung der Erkrankung Osteochondrose in den Grenzen eines großen finanziellen Aufwands, der aber auch einen großen züchterischen Gewinn (einstufige Selektion) mit sich bringt, oder einem geringeren finanziellen Aufwand, der aber auch zu einem geringeren züchterischen Erfolg führt (zweistufige Selektion), bewegt.

\subsubsection{Einfluss der Selektionsgewichte auf die Selektion gegen OC und die Zuchtwerte der Leistungsmerkmale}

Die Selektionsintensität innerhalb des Selektionsprogramms kann durch die Angabe von Selektionsgewichten variiert werden. Um sich einer optimalen Gewichtung der Merkmale anzunähern, wurden verschiedene Selektionsgewichte angewendet. 
Die Gewichtung der Leistungsmerkmale bleibt konstant, während die Gewichtung des Merkmals OC von keiner Berücksichtigung (Gewichtung 0) über eine Gewichtung, die den Heritabilitäten entspricht (Gewichtung 0,2), gleicher Gewichtung wie bei den Leistungsmerkmalen (Gewichtung 0,4) bis zu einer verstärkten Gewichtung der OC innerhalb des Selektionsindexes (Gewichtung 0,8) verändert wird. Die Gewichtung der Leistungsmerkmale und der OC mit: Dressur: 0,4/, Springen: 04/ und OC 0,2 folgt den Heritabilitäten der Merkmale, dürfte aber auch die reale Gewichtung der Merkmale zueinander in der Pferdezucht widerspiegeln, da bei Selektionsentscheidungen ein größeres Gewicht auf die Leistungsmerkmale als auf Gesundheitsmerkmale gelegt wird; dabei sind bei der Zuchtzieldefinition Leistungs- und konstitutionelle Merkmale als gleich wichtig anzusehen (PHILIPSSON, 1999). Durch die Anwendung der verschiedenen Selektionsszenarien stellte sich anhand der Ergebnisse die Gewichtung der Merkmale mit Dressur: 0,4/, Springen: 0,4/, OC: 0,2 als optimal heraus, die dann auch bei der zweistufigen Selektion übernommen wurde.

Die veränderten Selektionsgewichte, welche die Selektionsschärfe gegen OC bestimmen, haben auch Einfluss auf die Zuchtwerte der Leistungsmerkmale Dressur und Springen, wie Tabelle 25 verdeutlicht:

Tabelle 25: Veränderungen der Zuchtwerte für Dressur und Springen bei Anwendung unterschiedlicher Selektionsgewichte gegen OC (einstufige Selektion, genetisches Modell I)

\begin{tabular}{|lcc|cc|}
\hline \multicolumn{2}{|c|}{ Selektionsgewichte auf } & \multicolumn{2}{c|}{ Mittelwerte der Zuchtwerte } \\
Dressur & Springen & OC & Dressur ${ }^{\text {1) }}$ & Springen ${ }^{2)}$ \\
\hline 0,4 & 0,4 & 0,0 & 1,738 & 1,780 \\
\hline 0,4 & 0,4 & 0,2 & 1,709 & 1,748 \\
\hline 0,4 & 0,4 & 0,4 & 1,698 & 1,701 \\
\hline 0,4 & 0,4 & 0,8 & 1,642 & 1,678 \\
\hline
\end{tabular}

Die Ergebnisse zeigen, dass analog zu den unterschiedlichen Selektionsgewichten gegen OC Veränderungen bei den Zuchtwerten der Leistungsmerkmale eintreten. Der meiste Fortschritt wird bei Anwendung der Gewichtungen ohne Berücksichtigung der OC erreicht. 
Die Zuchtwerte von Dressur und Springen sinken bis zur verstärkten Gewichtung für $\mathrm{OC}$ ab, hier wird der geringste Fortschritt erreicht. Eine erhöhte Reduzierung der OC-Frequenz, die durch eine verstärkte Gewichtung des Merkmals OC erreicht wird, führt also bei den Zuchtwerte der anderen Merkmale, die ebenfalls in dieser Selektion berücksichtigt werden, zu geringeren Fortschritten.

\subsubsection{Einfluss der Remontierungsraten auf die OC-Frequenz und die Zuchtwerte der Leistungsmerkmale}

Ebenso wie die Veränderungen, die aus der Anwendung verschiedener Selektionsszenarien resultieren, sollten auch die Veränderungen, die durch die Angabe verschiedener Remontierungsraten entstehen, bewertet werden. Hinsichtlich der Reduzierung der OC-Frequenz werden die größten Fortschritte bei Anwendung einer Remontierungsrate von 0,05 auf der ersten Selektionsstufe erreicht, aus der sich programmintern eine Remontierungsrate von 0,2 auf der zweiten Selektionsstufe errechnet.

Das zweistufige Selektionsmodell simuliert das Auswahlverfahren bei der Hengstkörung, wie es in der praktischen Pferdezucht angewendet wird. Die erste Selektionsstufe stellt den der Körung vorgeschalteten Vorauswahl-Termin dar, bei dem eine Überprüfung der Hengste hinsichtlich ihrer Dressur und Springqualitäten stattfindet. Die Hengste, die hier eine überragende Leistung gezeigt haben, werden zur Körung zugelassen und bei diesem Termin nochmals einer Prüfung in den Disziplinen Dressur und Springen sowie einer röntgenologischen Überprüfung hinsichtlich ihres OC-Status unterzogen. Tabelle 26 zeigt die Veränderungen bei Anwendung unterschiedlicher Remontierungsraten hinsichtlich der Reduktion der OC-Frequenz und der Fortschritte in den Zuchtwerten der Leistungsmerkmale auf. 
Tabelle 26: Mittelwerte der OC-Frequenzen und der Zuchtwerte der Leistungsmerkmale bei Anwendung der zweistufigen Selektion mit unterschiedlichen Remontierungsraten

(genetisches Modell II mit zwei Klassen)

\begin{tabular}{|c|c|c|c|c|}
\hline \multicolumn{2}{|c|}{ Remontierungsrate $^{1)}$} & \multicolumn{3}{|l|}{ OC- } \\
\hline Erste Stufe & Zweite Stufe & Frequenz ${ }^{2) 3 \text { ) }}$ & Dressur ${ }^{4)}$ & Springen $^{5)}$ \\
\hline 0,01 & 1 & 0,205 & 1,763 & 1,770 \\
\hline 0,02 & 0,5 & 0,194 & 1,721 & 1,766 \\
\hline 0,05 & 0,2 & 0,183 & 1,662 & 1,745 \\
\hline 0,1 & 0,1 & 0,183 & 1,662 & 1,745 \\
\hline \multicolumn{5}{|c|}{ 1) Selektionsgewichte: Dressur 0,4; Springen 0,$4 ;$ OC 0,2} \\
\hline $\begin{array}{l}\text { 2) Durchschnittsfre } \\
\text { 4) Mittlerer Standar }\end{array}$ & $\begin{array}{l}\mathrm{OC} \text { in der ersten Genera } \\
0.004\end{array}$ & $\begin{array}{l}\text { ation }=0,2 \text { Mittlere } \\
\text { 5) Mittlerer }\end{array}$ & ndardfehler $=0,0$ & \\
\hline
\end{tabular}

Anhand der Ergebnisse wird deutlich, dass die Anwendung einer Remontierungsrate von 0,05 auf der ersten und 0,2 auf der zweiten Stufe unter Annahme gleicher ökonomischer Gewichtung der Merkmale zur größten Reduktion der OC-Frequenz führt. Die Selektionserfolge bei der Reduzierung der OC-Frequenz, die durch die Anwendung von Remontierungsraten erzielt werden, sind jedoch auch abhängig von der ökonomischen Gewichtung der OC, wie Tabelle 34 zeigt.

Hinsichtlich der Zuchtwerte der Leistungsmerkmale Dressur und Springen werden die größten Fortschritte bei Anwendung einer Remontierungsrate von 0,01 auf der ersten und 1 auf der zweiten Stufe erreicht. Diese Fortschritte lassen sich damit erklären, dass mit der Anwendung einer geringen Remontierungsrate von 0,01 auf der ersten Stufe sehr scharf in den Merkmalen Dressur und Springen selektiert wird, während auf der zweiten Stufe mit einer Remontierungsrate von 1 keine Selektion bei dem Merkmal Osteochondrose erfolgt. Diese Fortschritte werden mit abnehmender Selektionsschärfe für Dressur und Springen und mit zunehmender Selektionsschärfe für $\mathrm{OC}$ innerhalb der nächsten angewandten Remontierungsraten geringer, bis bei der Anwendung der Remontierungsraten 0,05 auf der ersten und 0,2 auf der zweiten Selektionsstufe die geringsten Fortschritte in den Zuchtwerten für Dressur und Springen erreicht werden. 


\subsection{Bewertung verschiedener simulierter Selektions- szenarien gegen $\mathrm{OC}$}

Mit Hilfe der vorgestellten genetischen Modelle und Selektionsmethoden konnten verschiedene Selektionsszenarien gegen OC simuliert werden, die in der praktischen Pferdezucht Anwendung finden oder finden könnten, wie die in Zukunft eventuell mögliche Diagnose der OC anhand eines Gentests oder die verstärkte Einbeziehung der Stuten in die Selektion gegen diese Erkrankung.

Bezüglich der Simulation der Selektion der Hengste für die Körung, wie sie mit der zweistufigen Selektion verfolgt wird, fand eine Weiterentwicklung des genetischen Modells II mit zwei phänotypischen Klassen statt, um die Effizienz verschiedener Auswahlmethoden bei der Hengstkörung bezüglich ihrer Effizienz bei der Reduzierung der OC Frequenz $\mathrm{zu}$ überprüfen und $\mathrm{zu}$ vergleichen. Auch die Veränderungen der Zuchtwerte der Leistungsmerkmale bei Anwendung dieser Auswahlmethoden wurden beobachtet und bewertet.

\subsubsection{Bewertung verschiedener simulierter Selektionsszenarien bei der Hengstkörung}

Das genetische Modell II mit vier phänotypischen Klassen stellt eine Weiterentwicklung des Modells mit zwei Klassen dar. Es erfolgte eine Verfeinerung der phänotypischen zwei Klassen in vier Klassen, die den vier offiziellen Röntgenbewertungsklassen nachempfunden sind. Diese vier Bewertungsklassen wurden 1985 von der internationalen Röntgenbewertungskommission erlassen, um einen Standard für die Einteilung und Bewertung von röntgenologischen Befunden zu schaffen. Das Spektrum dieses Bewertungsschemas reicht von „unbedeutend“ (Kategorie I) bis hin zur „deutlichen“ Abweichung (Kategorie IV) vom röntgenologischen Normalzustand mit vorausgesetzter negativer Leistungsbeeinflussung. Das zweistufige Selektionsverfahren wurde beibehalten, auf der ersten Stufe erfolgt die Selektion in den Merkmalen Dressur und Springen. Auf der zweiten Stufe erfolgt ebenfalls eine Selektion in den Leistungsmerkmalen und zusätzlich eine Selektion hinsichtlich des OC-Status. 
Aufgrund seiner röntgenologischen Befunde wird der Hengst einer der vier Röntgenklassen zugeordnet. Eine Einteilung in die Kategorie drei oder vier bedeutet, dass bei diesem Hengst erhebliche (Röntgenklasse drei) oder deutliche (Röntgenklasse vier) Abweichungen seiner röntgenologischen Befunde vom Normalzustand festgestellt wurden. Diese Einteilung kann bei diesem Modell durch überdurchschnittliche Leistungen ausgeglichen werden, so dass trotz erheblicher röntgenologischer Befunde ein positives Körurteil erfolgen kann.

Um die unterschiedlichen Selektionserfolge bei dem Ausgangsmodell mit zwei phänotypischen Klassen und vier phänotypischen Klassen zu vergleichen, werden sie in der folgenden Tabelle 27 gegenübergestellt:

Tabelle 27: OC-Frequenzen bei Darstellung der OC in zwei bzw. vier phänotypischen Klassen

\begin{tabular}{|c|c|c|c|}
\hline \multicolumn{2}{|c|}{ Remontierungsrate $^{1)}$} & \multicolumn{2}{|c|}{ OC-Frequenz $^{2)}$} \\
\hline Erste Stufe & Zweite Stufe & 4-Klassen ${ }^{3)}$ & 2-Klassen ${ }^{4)}$ \\
\hline 0,01 & 1 & 0,205 & 0,205 \\
\hline 0,02 & 0,5 & 0,145 & 0,194 \\
\hline 0,05 & 0,2 & 0,138 & 0,183 \\
\hline 0,1 & 0,1 & 0,138 & 0,183 \\
\hline
\end{tabular}

$\begin{array}{ll}3) \text { Mittlerer Standardfehler }=0,001 & \text { 4) } \text { Mittlerer Standardfehler }=0,001\end{array}$

Die Ergebnisse verdeutlichen, dass die Weiterentwicklung durch die Enteilung des Genotyps in vier, anstatt wie zuvor in zwei Klassen zu einem Selektionserfolg hinsichtlich der Reduktion der OC-Frequenz führt. Somit kann die Einteilung des normalverteilten Phänotyps in vier Klassen als positives Selektionsinstrument gewertet werden. Von SALDERN (2002) sieht bei der praktischen Anwendung der vier Röntgenklassen Probleme in der Form, dass nur noch Pferde mit den Röntgenklassen eins und zwei verkäuflich wären. Die Klassen drei und vier seien nahezu unverkäuflich, obwohl insbesondere in Klasse drei Pferde mit nicht allzu bedeutenden Röntgenbefunden eingestuft würden. Dieses Problem könne seiner Meinung nach mit der Schaffung von Zwischen- oder zusätzlichen Klassen abgemildert werden, um die Palette der Röntgenklassen für verkaufbare Pferde mit kleineren Befunden zu erweitern. 
Ein weiteres in der Pferdezuchtpraxis angewandtes Selektionsszenario bei der Körung ist die Ausschlussselektion. In dem simulierten Selektionsszenario ist OC ein normalverteiltes Merkmal, der Phänotyp für OC wird wie bei den anderen Modellen, die Auswahlverfahren bei der Körung simulieren, in vier Klassen eingeteilt. Im Vergleich $\mathrm{zu}$ den anderen Modellen (vier Klassen Modell, Modell mit Zuchtwertschätzung) findet hier jedoch keine Zuchtwertschätzung anhand von Eigen- oder Halbgeschwisterleistung statt, sondern ein Ausschluss von Hengsten bei der letzten Selektionsstufe der Körung. Die zweistufige Selektion wird beibehalten. Hengste, die auf der ersten Selektionsstufe überdurchschnittliche Leistungen gezeigt haben, werden auf der zweiten Stufe röntgenologisch untersucht und in die den Befunden entsprechende Röntgenklasse eingeteilt. An diesem Punkt fand eine Weiterentwicklung gegenüber dem Modell mit vier Klassen statt: zwei Ausschlussselektionsmodelle wurden entwickelt. Das eine Modell sieht einen Ausschluss von Hengsten mit den Röntgenklassen drei und vier von weiteren Selektionsentscheidungen vor. Bei dem zweiten Modell erfolgt der Ausschluss von Hengsten mit der Röntgenklasse vier von der Zucht.

Tabelle 28: OC-Frequenzen bei Anwendung der Ausschlussselektionsmodelle

\begin{tabular}{|c|c|c|c|}
\hline \multicolumn{2}{|c|}{ Remontierungsrate $^{1)}$} & \multicolumn{2}{|c|}{ OC-Frequenz $^{2)}$} \\
\hline & & Ausschluss & Ausschluss \\
\hline & & Röntgenklasse & Röntgenklasse \\
\hline Erste Stufe & Zweite Stufe & $\mathbf{I I I}+\mathbf{I V}^{3)}$ & $I^{4)}$ \\
\hline 0,01 & 1 & 0,205 & 0,205 \\
\hline 0,02 & 0,5 & 0,160 & 0,174 \\
\hline 0,05 & 0,2 & 0,142 & 0,160 \\
\hline 0,1 & 0,1 & 0,142 & 0,160 \\
\hline
\end{tabular}

2) Durchschnittsfrequenz für OC in der ersten Generation $=0,2$

4) Mittlerer Standardfehler $=0,001$

Erwartungsgemäß ist eine vermehrte Reduktion der OC-Frequenz bei Anwendung des Modells, bei dem ein Ausschluss der Hengste erfolgt, die aufgrund ihrer röntgenologischen Befunde in die Röntgenklasse drei und vier eingeteilt wurden, zu verzeichnen. 
Da die Ausschlussmodelle einen definitiven Ausschluss von Hengsten aus der Zucht vorsehen, sollte eine besondere Beobachtung der Zuchtwerte der Leistungsmerkmale bei Anwendung dieser Modelle gewährleistet sein:

Tabelle 29: Mittlere Zuchtwerte der Leistungsmerkmale bei Anwendung der Ausschlussselektionsmodelle

\begin{tabular}{|c|c|c|c|}
\hline Remon & rungsrate $^{1)}$ & Mittelwerte deı & Zuchtwerte bei: \\
\hline Erste Stufe & Zweite Stufe & $\begin{array}{l}\text { Ausschluss } \\
\text { Röntgenklasse } \\
\text { III+IV } \\
\text { Dressur }^{2)} \text { Springen }\end{array}$ & $\begin{array}{c}\text { Ausschluss } \\
\text { Röntgenklasse } \\
\text { IV } \\
\text { Dressur }^{4)} \text { Springen }\end{array}$ \\
\hline 0,01 & 1 & $1,658 \quad 1,706$ & $1,682 \quad 1,715$ \\
\hline 0,02 & 0,5 & 1,641 & 1,663 \\
\hline 0,05 & 0,2 & 1,671 & 1,649 \\
\hline 0,1 & 0,1 & 1,632 & 1,649 \\
\hline
\end{tabular}

Bezüglich der Fortschritte in den Zuchtwerten der Leistungsmerkmale schneidet das Modell mit Ausschluss der Röntgenklasse vier besser ab. Das Modell mit Ausschluss der Röntgenklassen drei und vier führt zu geringeren Fortschritten in Dressur und Springen. Bei diesem Modell ist diese Tendenz mit besonderer Vorsicht zu bewerten, da hier ein Ausschluss von potentiellen Vatertieren aus der Zucht erfolgt. Mögliche positive Eigenschaften des Hengstes bezüglich der Leistungsmerkmale, die einen eventuellen Zuchtfortschritt in diesen Merkmalen hätten bringen können, gehen durch den Ausschluss von der Zucht verloren.

Da das letztendliche Ziel dieser Arbeit sein soll, Empfehlungen hinsichtlich der Selektion gegen OC auszusprechen, soll an dieser Stelle ein Vergleich zwischen der Ausschlussselektion und dem Modell mit vier Klassen erfolgen, da beide Selektionsszenarien in der Praxis bei der Hengstkörung Anwendung finden. Die Modelle werden hinsichtlich ihrer Effizienz bei der Reduzierung der OC Frequenz und den Veränderungen die sie bei den Zuchtwerten der Leistungsmerkmale auslösen, in Tabelle 30 verglichen. 
Tabelle 30: OC-Frequenzen bei Anwendung des Modells mit vier Klassen ohne Ausschluss und der Anwendung der Ausschlussselektionen mit Ausschluss der Röntgenklasse IV/Ausschluss der Röntgenklassen III und IV

\begin{tabular}{|c|c|c|c|c|}
\hline Remol & Ingsrate $^{1)}$ & & OC-Frequen & \\
\hline Erste Stufe & Zweite Stufe & $\begin{array}{l}\text { 4-Klassen } \\
\text { Modell }^{3)}\end{array}$ & $\begin{array}{c}\text { Ausschluss } \\
\text { Röntgenkl. } \\
\text { IV }\end{array}$ & $\begin{array}{l}\text { Ausschluss } \\
\text { Röntgenkl. }^{5)} \\
\text { III+IV }\end{array}$ \\
\hline 0,01 & 1 & 0,205 & 0,205 & 0,205 \\
\hline 0,02 & 0,5 & 0,145 & 0,174 & 0,160 \\
\hline 0,05 & 0,2 & 0,138 & 0,160 & 0,142 \\
\hline 0,1 & 0,1 & 0,138 & 0,160 & 0,142 \\
\hline $\begin{array}{l}\text { 1) angewendete } S \\
\text { 2) Durchschnittsfr }\end{array}$ & $\begin{array}{l}\text { ewichte: Dressur 0, } \\
\text { ir OC in der ersten }\end{array}$ & $\begin{array}{l}\text { ringen } 0,4, O C \\
\text { ation }=0,2\end{array}$ & rer Sta & 0,001 \\
\hline 4) mittlerer Standa & $=0,001$ & & ittlerer Standardfe &, 002 \\
\hline
\end{tabular}

Die Ergebnisse zeigen, dass zwischen dem Modell mit vier Klassen und dem Modell mit Ausschluss der Röntgenklasse vier ein deutlicher Unterschied hinsichtlich der Reduzierung der OC-Frequenz festgestellt werden kann (0,138 vs. 0,160). Beim Vergleich des vier-Klassen-Modells mit dem Modell, dass einen Ausschluss der Röntgenklassen drei und vier vorsieht, ist hingegen kein großer Unterschied festzustellen, (0,138 vs. 0,142 bei Anwendung der Remontierungsraten 0,05 auf der ersten und 0,2 auf der zweiten Selektionsstufe). Dieser geringe Unterschied zwischen den beiden Modellen hinsichtlich der Reduktion der OC-Frequenz ist darauf zurückzuführen, dass bei der Selektion in beiden Modellen eine geringe Remontierungsrate angewandt wird, die eine scharfe Selektion auf beiden Selektionsstufen bedeutet. Dies führt dazu, dass auf der zweiten Selektionsstufe nur noch mit einer sehr geringen Wahrscheinlichkeit OC positive Hengste vorhanden sein werden. 
Bei Selektionsentscheidungen sollten jedoch die Veränderungen in den Zuchtwerten der Leistungsmerkmale nicht außer Acht gelassen werden.

Tabelle 31: Mittlere Zuchtwerte der Leistungsmerkmale bei Anwendung des Modells mit vier Klassen ohne Ausschluss und der Anwendung der Ausschlussselektionen mit Ausschluss der Röntgenklasse IV/Ausschluss der Röntgenklassen III und IV

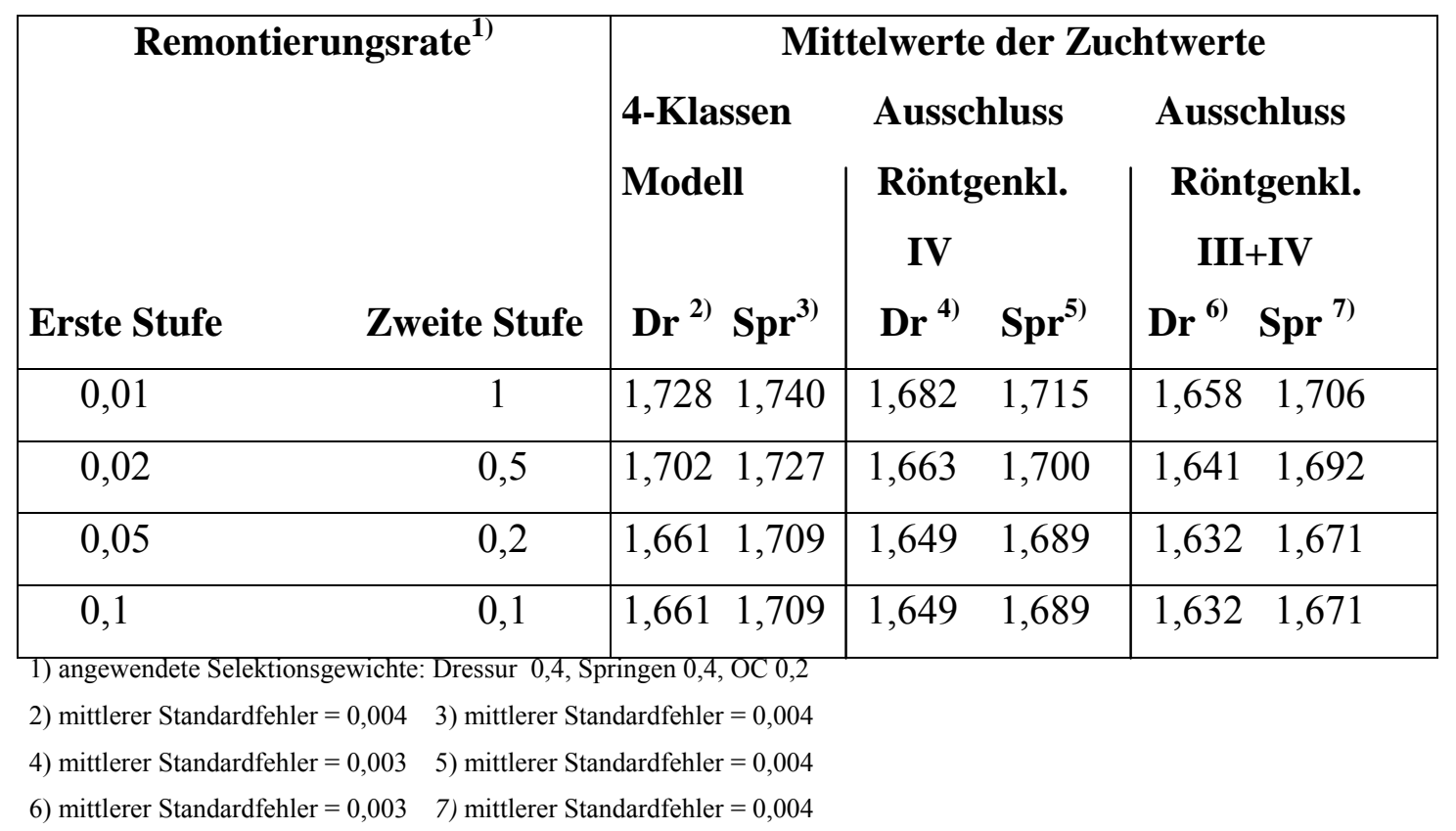

Auch hinsichtlich der Veränderungen in den Zuchtwerten der Leistungsmerkmale sind im Vergleich der Selektionsszenarien bei Anwendung des Modells mit vier Klassen die größten Fortschritte zu verzeichnen.

Die Anwendung eines OC-positiven Hengstes in der Zucht, die aus der Anwendung des Modells mit vier Klassen resultieren könnte, ist vom Standpunkt aus, ein gesundes Pferd erzeugen zu wollen, kritisch zu beurteilen. Das Zuchtziel in der deutschen Reitpferdezucht zielt jedoch auf ein in den Leistungsmerkmalen begabtes und im Turniersport einsetzbares Pferd ab; aus diesem Blickwinkel birgt die Anwendung des Ausschlussmodells die Gefahr, möglicherweise wichtige Eigenschaften eines Hengstes bezüglich des Zuchtfortschritts in den Leistungsmerkmalen Dressur und Springen für die Pferdezucht zu verlieren. 
Diese Überlegungen verdeutlichen, dass es wichtig ist, die Leistungen in Dressur und Springen und den röntgenologischen Status eines Hengstes im Zusammenhang zu betrachten und anhand dieser Informationen abzuwägen zwischen den möglichen positiven Einflüssen auf den Zuchtfortschritt hinsichtlich der Leistungsmerkmale und den möglichen negativen Einflüssen durch einen OC-Befall.

Weitere Sicherheit für Selektionsentscheidungen könnte an dieser Stelle der Einsatz einer Zuchtwertschätzung für OC bringen. Dieses Szenario wurde aufbauend auf dem genetischen Modell II mit vier phänotypischen Klassen simuliert. Hier werden anhand der Eigen- und der väterlichen Halbgeschwisterleistung eines Hengstes bei den Leistungsmerkmalen und für den OC-Status, Zuchtwerte ermittelt, anhand derer dann die Selektion erfolgt. Die Anzahl der Halbgeschwister kann angegeben werden. Es wurden Berechnungen mit der Angabe von 5, 20 und 40 Halbgeschwistern angestellt. Erwartungsgemäß ist die höchste Reduzierung der OC-Frequenz bei der Berechnung mit 60 Halbgeschwistern zu erzielen. Da eine Anzahl von 20 väterlichen Halbgeschwistern eines Hengstes innerhalb eines Körjahrganges jedoch als realistische Angabe erscheint, werden die Ergebnisse mit dieser Anzahl Halbgeschwister zum Vergleich mit den anderen Selektionsszenarien verwendet. Um eine Aussage über die Effizienz dieses Modells im Vergleich zu den beiden bereits vorgestellten Modellen treffen zu können, sind die Ergebnisse aller drei Modelle bezüglich der Reduktion der OC-Frequenz in Tabelle 32 dargestellt.

Tabelle 32: OC-Frequenzen bei Anwendung verschiedener Selektionsverfahren für Hengste

\begin{tabular}{|c|c|c|c|c|}
\hline \multicolumn{2}{|c|}{ Remontierungsrate $^{1)}$} & \multicolumn{3}{|c|}{ OC-Frequenz $^{2)}$} \\
\hline Erste Stufe & Zweite Stufe & $\begin{array}{l}\text { 4- Klassen } \\
\text { Modell }^{5)}\end{array}$ & $\begin{array}{l}\text { Ausschluss } \\
\text { Modell }^{\text {3) 6) }}\end{array}$ & $\begin{array}{l}\text { ZWS } \\
\text { Modell }^{4)} \text { 7) }\end{array}$ \\
\hline 0,01 & 1 & 0,205 & 0,205 & 0,205 \\
\hline 0,02 & 0,5 & 0,145 & 0,160 & 0,139 \\
\hline 0,05 & 0,2 & 0,138 & 0,142 & 0,124 \\
\hline 0,1 & 0,1 & 0,138 & 0,142 & 0,124 \\
\hline
\end{tabular}
2) Durchschnittsfrequenz für OC in der ersten Generation $=0,2$
3) Röntgenklassen III und IV ausselektiert
4) ZWS anhand von $20 \mathrm{HG}$
5) mittlerer Standardfehler $=0,001$
6) mittlerer Standardfehler $=0,002$
7) mittlerer Standardfehler $=0,002$ 
Anhand der Ergebnisse wird deutlich, dass mit der Anwendung des Zuchtwertschätzmodells im Vergleich zu den beiden anderen Modellen, die größte Reduzierung der OC-Frequenz erreicht werden kann. Die OC-Frequenz sinkt auf einen Wert von 0,124 im Vergleich zum Wert 0,138, der bei der Anwendung des Vier-Klassen-Modells in der fünften Generation erreicht wird. Das Ausschlussmodell platziert sich mit einem Wert von 0,142 dahinter.

Die Veränderungen in den Zuchtwerten der Leistungsmerkmale bei Anwendung der verschiedenen Selektionsszenarien sind in der folgenden Tabelle 33 dargestellt.

Tabelle 33: Mittlere Zuchtwerte der Leistungsmerkmale bei Anwendung verschiedener Selektionsverfahren für Hengste

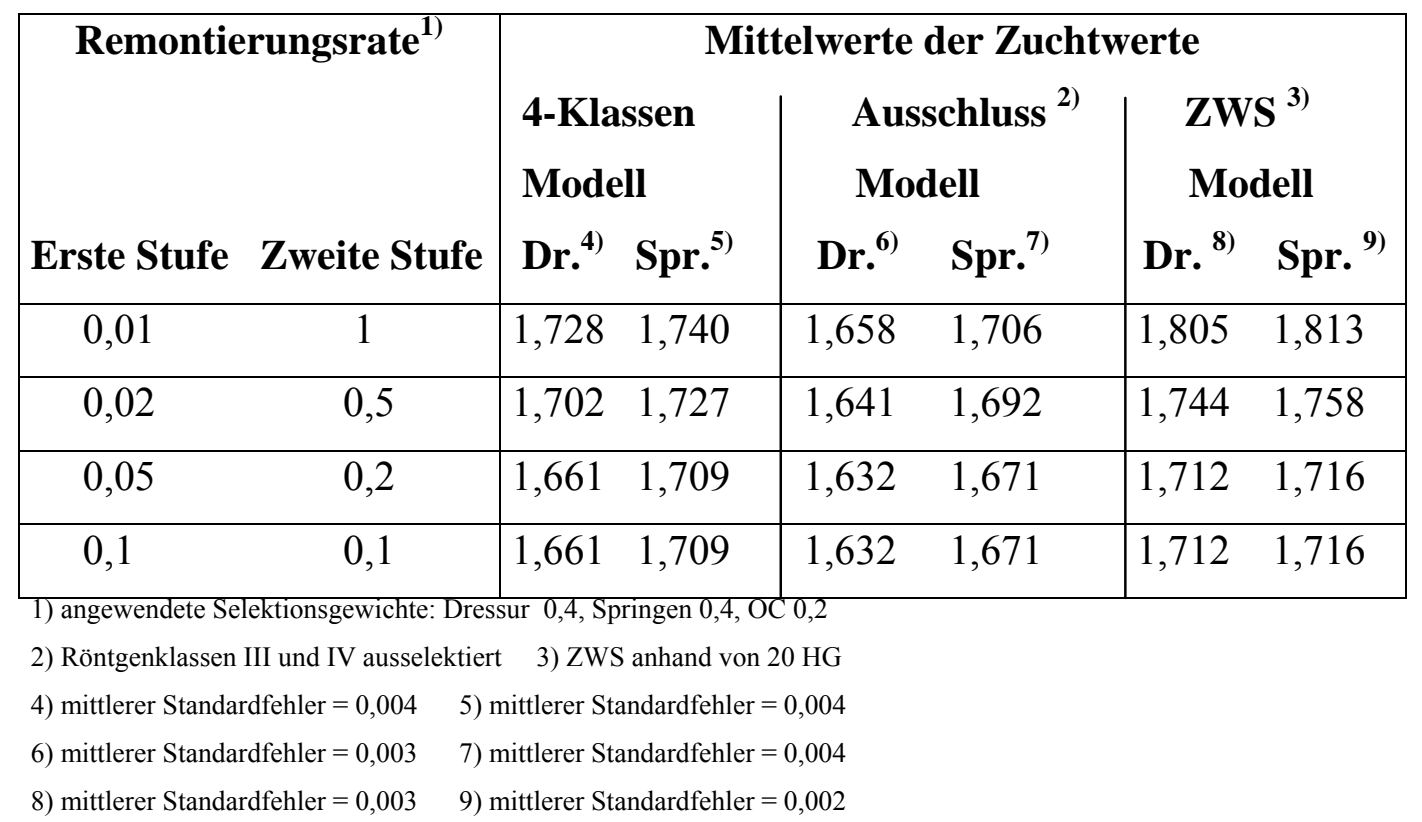

Auch an dieser Stelle werden die größten Fortschritte bei der Anwendung des Modells mit Zuchtwertschätzung gemacht (1,712 als Durchschnitt für das Merkmal Dressur und 1,716 für das Merkmal Springen in der fünften Generation bei Anwendung der Remontierungsraten 0,05 auf der ersten und 0,2 auf der zweiten Selektionsstufe). Das Modell mit vier Klassen platziert sich mit seinen Fortschritten im Vergleich dahinter (1,661 als Durchschnitt für das Merkmal Dressur und 1,709 für das Merkmal Springen in der fünften Generation bei Anwendung der gleichen Remontierungsraten); während mit dem Ausschlussmodell der geringste Fortschritt in den Leistungsmerkmalen erzielt wird (1,632 als Durchschnitt für das Merkmal Dressur und 1,671 für das Merkmal Springen in der fünften Generation bei Anwendung der gleichen Remontierungsraten). 
Somit kann als Zusammenfassung der erzielten Ergebnisse der simulierten Selektionsszenarien der Hengstkörung festgestellt werden, dass sowohl bei Reduktion der OC-Frequenz, als auch bei den Zuchtwerten der Leistungsmerkmale der größte Selektionserfolg bei Anwendung des Modells mit Zuchtwertschätzung erfolgt.

Um die drei vorgestellten Selektionsmodelle auch hinsichtlich ihres zu erwartenden Zuchtfortschritts bei der Integration in Zuchtprogramme bewerten zu können, würde der Gesamtzuchtfortschritt $(\Delta \mathrm{T})$ berechnet, der sich definiert als die Summe der Zuchtfortschritte der einzelnen Merkmale $\left(\Delta \mathrm{T}_{1-3}\right)$, multipliziert mit der relativen ökonomischen Gewichtung $\left(\mathrm{v}_{\mathrm{i}}\right)$ der Merkmale. Da sich für fast alle Merkmale der Reitpferdezucht keine ökonomischen Gewichtungen ableiten lassen (SCHÜLER et al., 2001) wurden verschiedene ökonomische Gewichtungen der Leistungsmerkmale Dressur und Springen und der Osteochondrose angenommen. In diesem Zusammenhang wäre eine Erfassung und Einordnung des tatsächlichen wirtschaftlichen Verlustes ausgehend von der Osteochondrose notwendig, um die Implementierung der verschiedenen Selektionsmethoden auch im Hinblick auf die ökonomischen Auswirkungen richtig bewerten zu können.

Tabelle 34: Gesamtzuchtfortschritte der Selektionsmodelle bei unterschiedlichen ökonomischen Gewichtungen der Merkmale

\begin{tabular}{|c|c|c|c|c|c|}
\hline Gewichtung 1) & \multicolumn{2}{|c|}{ Remontierungsrate } & \multirow{2}{*}{$\begin{array}{c}\text { 4-Klassen- } \\
\text { Modell }\end{array}$} & \multirow{2}{*}{$\begin{array}{c}\text { Ausschluss- }^{2)} \\
\text { Modell }\end{array}$} & \multirow{2}{*}{$\begin{array}{l}\text { ZWS- }^{3} \\
\text { Modell }\end{array}$} \\
\hline Dr. Spr. OC & 1. Stufe & 2.Stufe & & & \\
\hline 0,2 & 0,01 & 1 & 0,692 & 0,671 & 0,722 \\
\hline & 0,02 & 0,5 & 0,708 & 0,683 & 0,725 \\
\hline & 0,05 & 0,2 & 0,699 & 0,684 & 0,716 \\
\hline & 0,1 & 0,1 & 0,699 & 0,684 & 0,716 \\
\hline \multirow[t]{4}{*}{$\begin{array}{lll}0,33 & 0,33 & 0,33\end{array}$} & $\overline{0,0,01}$ & 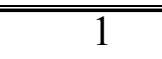 & $\bar{~} 1,154$ & 1,120 & $1,1,204$ \\
\hline & 0,02 & 0,5 & 1,161 & 1,124 & 1,188 \\
\hline & 0,05 & 0,2 & 1,144 & 1,120 & 1,168 \\
\hline & 0,1 & 0,1 & 1,144 & 1,120 & 1,168 \\
\hline
\end{tabular}

3) ZWS anhand von $20 \mathrm{HG}$ 
Bei der Bewertung der Gesamtzuchtfortschritte setzt sich die zuvor anhand der Ergebnisse ermittelte Tendenz fort, denn auch hier führt das Modell mit Zuchtwertschätzung zu den größten Fortschritten, gefolgt vom vier Klassen Modell, das Ausschlussmodell führt zu den geringsten Fortschritten. Innerhalb der beiden Gewichtungsalternativen zeigt sich, dass die Remontierungsraten auf der ersten und zweiten Stufe von 0,02 und 0,5 die höchsten Gesamtzuchtfortschritte liefern.

Je höher das relative ökonomische Gewicht auf OC im Vergleich zu Dressur und Springen ist, desto schärfer sollte auf der zweiten Stufe unter Nutzung von Informationen zum OC-Status selektiert werden. Die Selektionsschärfe gegen Osteochondrose hängt ab vom gegebenen Status und dem erwünschten Ziel; und ist somit für jede Population spezifisch.

Bei den ermittelten Ergebnissen wurden keine Korrelationen zwischen OC und den Leistungsmerkmalen angenommen. Korrelationen zwischen orthopädischen Befunden und den gängigen Selektionskriterien sind für züchterische Fragen relevant, da sie mitunter die tatsächliche Bedeutung der OC für ein Zuchtprogramm umreißen und Auskunft darüber geben, ob eine verstärkte Selektion auf bestimmte Parameter negative Auswirkungen auf die Gliedmaßengesundheit in der Population hat. Es wurden zahlreiche Studien durchgeführt, um die Beziehung zwischen Gliedmaßenerkrankungen und den Kriterien der Leistung beim Pferd zu analysieren. WINTER (1995) und WILLMS (1988) berechneten genetische und phänotypische Korrelationen zwischen OCD und Leistungsprüfungen. WINTER (1995) ermittelte zwar neutrale phänotypische Korrelationen, stellte aber aufgrund der relativ hohen positiven genetischen Korrelationen zwischen OCD und Merkmalen der Hengstleistungsprüfung (HLP) fest, dass mit einer Verbesserung der Noten der Rittigkeit eine Zunahme im Schweregrad der Befunde einhergeht und deutete somit eine leicht ungünstige Beziehung an, die bei zunehmender Selektion auf Leistung auf einen Anstieg der Häufigkeit von Gliedmaßenbefunden schließen lässt. WILLMS (1988) berechnete anhand seiner Untersuchung an ähnlichen Merkmalen im Rahmen einer Eigenleistungsprüfung von Stuten auf Station neutrale Beziehungen zwischen OCD und den Grundgangarten. 
Bei den Merkmalen Freispringen und Rittigkeit lagen sie in einem leicht positiven Bereich von 0,14, so dass sich hier eine leicht positive genetische Beziehung ergibt, die sich aber aufgrund der hohen Standardfehler, die auf geringe Tierzahlen begründet werden können, statistisch nicht absichern lässt. RICARD et al. (2002) untersuchten die Auswirkungen von positiven röntgenologischen Befunden auf die Leistung von 733 französischen Warmblütern. Die Autoren konnten hier einen wenig einheitlichen Einfluss feststellen und deuten an, dass Pferde mit extremen Befunden schlechtere Leistung erbracht haben. In einer Studie von BREHM und STAECKER (1994) wurden die Rennleistungen OC-positiver und OC-negativer Trabrennpferde verglichen. Die im Rahmen dieser Studie beobachteten Pferde wiesen jeweils einen OC-Befall im Sprunggelenk auf. Die Untersuchung brachte das Ergebnis, dass sich die Leistungen der beiden Gruppen in Bezug auf Merkmale wie Anzahl Starts, Siege und Platzierungen sowie Kilometerrekord nicht signifikant unterschieden und somit keine Beeinträchtigung der Leistung durch OC festzustellen war. Auch SCHOBER (2003) konnte anhand der Röntgenbefunde von 629 hannoverschen Fohlen und deren Müttern, die in Beziehung zu Leistungskriterien gesetzt wurden, keine Hinweise auf eine Beeinträchtigung der verschiedenen Leistungsparameter (Dressur und Springen) bei verstärkter Berücksichtigung der Gesundheit bezüglich OC feststellen. STOCK (2004) simulierte die Einbeziehung von Röntgenbefunden in Selektionsentscheidungen und kam $\mathrm{zu}$ dem Ergebnis, dass aufgrund des nur verlangsamten Zuchtfortschritts im Hinblick auf die Leistungszuchtwerte, eine gleichzeitig auf Leistung und Röntgenbefunden basierende Selektion praktikabel ist.

Vergleichbare Simulationsstudien zur Untersuchung der Auswirkungen von Selektionsstrategien gegen $\mathrm{OC}$ durch Computersimulationen wurden von KOENEN et al. (2000) und PIERAMATI et al. (2003) durchgeführt. In der niederländischen Warmblutzucht wird seit 1987 der OC-Status eines Hengstes bei Selektionsentscheidungen im Rahmen der Körung berücksichtigt. Hengste mit osteochondrotischen Veränderungen in Sprung- und/oder Kniegelenken, Arthrose im Fesselgelenk, Spat/und oder Podotrochlose werden von der weiteren Selektion ausgeschlossen und können an der letzten Selektionsstufe nicht mehr teilnehmen. Diese und andere Selektionsmethoden wurden von KOENEN et al. (2000) hinsichtlich ihrer Effizienz bei der Reduzierung der OC-Frequenz anhand einer Computersimulationsstudie untersucht. 


\section{Diskussion}

Die simulierte Population bestand hier aus 15.000 Zuchtstuten und 200 Zuchthengsten. Es wurde eine OC-Ausgangsfrequenz von 25\% angenommen, deren Veränderungen über einen Zeitraum von 50 Jahren beobachtet wurden. Die Heritabilität der Osteochondrose wurde mit einem Wert von $\mathrm{h}^{2}=0,2$ angegeben.

Auch in Italien wird in der Reitpferdezucht eine Selektion gegen OC durchgeführt: ein OC-Befall führt hier ebenfalls zum Ausschluss des Pferdes aus der Zucht. PIERAMATI et al. (2003) beobachteten die Veränderungen der OC-Frequenz bei Anwendung verschiedener Selektionsstrategien innerhalb der italienischen Marremanno Population. Die Röntgenuntersuchungen von 350 Pferden (165 Hengste und 185 Stuten), die von 75 Hengsten abstammten bildeten die Grundlage für diese Untersuchung. Die Erfassung der Veränderungen der OC-Ausgangsfrequenz, die mit $16 \%$ angenommen wurde, erfolgte über fünf Generationen. Die Heritabilität für OC wurde mit den Werten $\mathrm{h}^{2=} 0,09$ und $\mathrm{h}^{2=} 0,14$ in die Berechnungen einbezogen.

In beiden Untersuchungen wurde $\mathrm{OC}$ als ein Ja-oder-Nein Merkmal mit einer zugrundegelegten genetischen Normalverteilung beschrieben, das dem hier in dieser Studie entwickelten genetischen Modell II mit zwei phänotypischen Klassen entspricht.

KOENEN et al. (2000) untersuchten in ihrer Simulationsstudie die Auswirkungen folgender Selektionsszenarien:

- keine Selektion auf OC

- Ausschluss OC-positiver Hengste

- (derzeitige Strategie in der niederländischen Warmblutzucht)

- Auswirkungen bei Einbeziehung von Nachkommen- und Verwandten Leistung

In der Studie von PIERAMATI et al. (2003) wurden folgende Szenarien simuliert:

- Selektion positiver Hengste bei der Prüfung auf Station

- Selektion beider Geschlechter

- Selektion positiver Hengste und Hengstmütter 
Tabelle 35 gibt einen Überblick über die erzielten Ergebnisse der Simulationsstudien von KOENEN et al. (2000) und PIERAMATI et al. (2003).

Tabelle 35: Ergebnisse vergleichbarer Simulationsstudien zur züchterischen Bekämpfung der Osteochondrose

\begin{tabular}{|c|c|c|}
\hline Autor & Selektionsstrategien & Erzielte Ergebnisse \\
\hline \multirow{4}{*}{$\begin{array}{l}\text { KOENEN et al. } \\
(2000)\end{array}$} & & $\begin{array}{l}\text { OC-Ausgangsfrequenz: } 25 \% \\
\text { Reduktion auf: } \\
\text { (bezogen auf } 50 \text { Jahre) }\end{array}$ \\
\hline & keine Selektion gegen OC & $25 \%$ \\
\hline & Selektion der Hengste & $17 \%$ \\
\hline & Nachkommenprüfung Hengste & $13 \%$ \\
\hline \multirow{4}{*}{$\begin{array}{l}\text { PIERAMATI et al. } \\
\text { (2003) }\end{array}$} & & $\begin{array}{l}\text { OC-Ausgangsfrequenz: } 16 \% \\
\text { Reduktion auf: } \\
\text { (bezogen auf } 5 \text { Generationen) }\end{array}$ \\
\hline & $\begin{array}{l}\text { positive Hengste bei Prüfung auf } \\
\text { Station }\end{array}$ & $5,8 \%$ \\
\hline & Selektion beider Geschlechter & $1,8 \%$ \\
\hline & $\begin{array}{l}\text { Selektion positiver Hengste und } \\
\text { Hengstmütter }\end{array}$ & $5,5 \%$ \\
\hline
\end{tabular}

Eine weitere bedeutende Erkrankung in der deutschen Warmblutpopulation ist die Hemiplegia laryngis (Kehlkopfpfeifen). Ebenso wie bei einem OC-Befall führt dieser gesundheitliche Defekt zu Problemen für die praktische Pferdezucht und ruft damit verbunden auch wirtschaftliche Nachteile hervor. MIESNER (1996) untersuchte anhand der laryngoskopischen Untersuchung von 240 Fohlen und 429 zwei- bis fünfjährigen Nachkommen von 20 untersuchten Hengsten die Frequenz dieses Merkmals innerhalb der Hannoveraner Population. Anhand der Ergebnisse konnte eine deutliche erbliche Komponente dieser Erkrankung festgestellt werden $\left(h^{2}=0,61\right)$. Weiterhin wurden in der Studie von MIESNER (1996) umweltbedingte Einflussfaktoren auf die Entstehung untersucht und mögliche Ansatzpunkte für die Integration der Selektion gegen diesen gesundheitlichen Defekt innerhalb der deutschen Reitpferdezucht angegeben. 
Eine Bewertung der bei den simulierten Auswahlverfahren auf der Hengstseite erzielten Ergebnisse innerhalb dieser Studie sollte aus verschiedenen Blickwinkeln erfolgen.

Die Beachtung von Gesundheitsaspekten ist eine häufig gestellte Forderung, da das allgemeine Zuchtziel Kriterien der Leistung und der Gesundheit gleichgewichtig betrachtet (BRUNS, 2001). Auch der Käuferwunsch zielt auf ein vielseitig einsetzbares und gesundes Sportpferd ab, um das Leistungspotential der Pferde voll ausschöpfen zu können. Die Maßgabe, gesunde, belastbare und langlebige Pferde zu züchten, sollte bei Selektionsentscheidungen konsequente Berücksichtigung finden. Dieser Forderung würde mit der Anwendung der Ausschlussselektion in Zuchtprogrammen Rechnung getragen werden, da Hengste mit einem röntgenologischen Status, der in die Röntgenklassen drei und vier eingeteilt wurde, konsequent von der Zucht ausgeschlossen werden. Diese Selektionsmethode wird in der niederländischen Warmblutzucht bei der Hengstkörung verwendet. KOENEN et al. (2000) berechneten für den Ausschluss von OC-positiven Hengsten aus der Zucht eine Reduzierung der OC-Frequenz von $25 \%$ auf $17 \%$, also um etwa $1,8 \%$ pro Generation. PIERAMATI et al. (2003) kamen unter Annahme des gleichen Selektionsszenarios bei einer Ausgangsfrequenz von 16\% und Angabe verschiedener Heritabilitäten (9\% und 14\%), zu einer Reduktion der OC-Frequenz auf 5,8\% innerhalb von fünf Generationen. Bei Anwendung der Ausschlussselektionsmethode ist eine genaue Beobachtung der Zuchtwerte der Leistungsmerkmale besonders wichtig, da hier Hengste mit möglicherweise wichtigen und nutzvollen Eigenschaften in Bezug auf die Leistungsmerkmale Dressur und Springen für die Zucht verloren gehen. Zu berücksichtigen gilt dabei auch, dass überdurchschnittliche Begabungen und Leistungen in diesen Merkmalen bei der Pferdevermarktung durch einen dementsprechenden Kaufpreis honoriert werden, und das Ziel jeden Pferdezüchters darin besteht, seine Zuchtprodukte gewinnbringend zu vermarkten. Eine Berücksichtigung der Fortschritte bei den Zuchtwerten der Leistungsmerkmale erfolgt bei dem Modell mit vier Klassen. Aus der Anwendung dieser Selektionsmethode kann aber auch die Verwendung OC-positiver Hengste in der Zucht resultieren. Hinsichtlich der möglichen negativen Auswirkungen auf eine Population ist der Einsatz eines OC-positiven Hengstes in der Zucht kritisch zu beurteilen. 
Die Höhe der Kosten, die durch röntgenologische Veränderungen entstehen können, die der Hengst an seine Nachkommen weiter geben kann, sind schwer abzuschätzen. Sie leiten sich vor allem aus Röntgen- und Operationskosten sowie einer möglichen Wertminderung bei der Vermarktung eines OC-positiven Pferdes ab. Auch die Frage, wer diese Kosten übernehmen soll, ist schwer zu klären.

Um die Einbeziehung beider Merkmalskomplexe (Leistung und Gesundheit) in Selektionsentscheidungen $\mathrm{zu}$ verwirklichen und den Verlust von positiven Eigenschaften hinsichtlich der Leistungsmerkmale zu vermeiden, sollte das Modell mit Zuchtwertschätzung bei der Integration der Selektion gegen Osteochondrose in Zuchtprogrammen zum Einsatz kommen. Bei dem aktuellen Modell werden Zuchtwerte anhand der Leistung aller vorhandenen Verwandten (im Wesentlichen ist das die Eigenleistung und die Leistung väterlicher Halbgeschwister) in den Merkmalen Dressur und Springen sowie des OC-Status ermittelt, anhand derer dann die Selektion erfolgt. Die Selektion basiert also nicht nur auf der Eigenleistung eines Probanden, sondern hier werden auch Informationen von Verwandten einbezogen, was eine größere Sicherheit und Genauigkeit der erzielten Ergebnisse erwarten lässt. Die Gewichtung der Eigenleistung ist gerade bei jungen Hengsten ein Diskussionspunkt. Zusatzinformationen bei Hengsten mit Eigenleistung sind erwünscht und notwendig zur frühzeitigen und genauen Zuchtwertschätzung (VELSEN-ZERWECK, 1998). Diese Erwartungen spiegeln sich bei den erzielten Ergebnissen wider: hinsichtlich der Reduzierung der OC-Frequenz und der Zuchtwerte der Leistungsmerkmale Dressur und Springen wurden hier die größten Fortschritte im Vergleich aller drei Modelle erzielt. Die Durchführung einer Zuchtwertschätzung für OC würde den Vorteil bieten, dass zum Zeitpunkt der

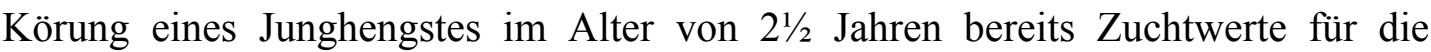
Merkmale Dressur, Springen und OC vorlägen, die weitere Sicherheiten bei Selektionsentscheidungen darstellen würden. Damit dieses Szenario in der Praxis funktionieren kann, müsste während des ersten Deckjahres eines gekörten Hengstes nach Ablegung seiner Leistungsprüfung, im Rahmen der integrierten Zuchtwertschätzung eine Zuchtwertschätzung für $\mathrm{OC}$ erfolgen. Für eine ausreichende Sicherheit der Zuchtwerte muss eine genügend große Anzahl von Verwandten-Informationen hinsichtlich OC vorhanden sein. 
Die Quelle, um diese Informationen zu erhalten, könnte auf der einen Seite die röntgenologische Überprüfung der Nachkommen eines Hengstes im Fohlen- oder Jungpferdealter sein. KOENEN et al. (2000) bezogen das Szenario einer Nachkommenüberprüfung in ihre Simulationsstudie ein. Sie kamen zu dem Ergebnis, dass sich die OC-Frequenz innerhalb von 50 Jahren von $25 \%$ auf $15 \%$ reduzieren ließe, was einer Abnahme von 2,8\% pro Generation entspräche. Bezüglich der röntgenologischen Darstellung von osteochondrotischen Veränderungen im Fohlenoder Jungpferdealter ermittelten DIK et al. (1999), dass Veränderungen am Sprunggelenk im Alter von fünf Monaten als endgültig angesehen werden können, beim Knie im Alter von acht Monaten. Die Relevanz von Röntgenbildern, die im Fohlenalter gemacht wurden, ist für KOENEN et al. (2000) im Hinblick auf Selektionsentscheidungen noch nicht geklärt, da die genetische Korrelation zwischen OC-Befunden im Fohlenalter und dem Alter von drei Jahren noch nicht bekannt sei. SCHOBER (2003) fand im Rahmen ihrer Untersuchung hohe Korrelationen für OCBefunde im Sprunggelenk zwischen dem Fohlenalter und dem Alter von zwei Jahren, für OC im Fesselgelenk wurden für die gleiche Zeitspanne geringere Korrelationen festgestellt. Der Vorteil einer Befunderhebung im Fohlenalter ist ein früher Informationsgewinn über Hengste im ersten Deckeinsatz. Auch HOPPE et al. (1985) und PHILIPSSON (1993) schlagen aufgrund der frühen Feststellbarkeit der Erkrankung einen Nachkommen-Test als Basis für die Selektion vor. Ein weiterer Ansatzpunkt könnte die Einbeziehung einer röntgenologischen Untersuchung auf OC-Befall in den Ablauf der Stutbuchaufnahme oder der Stutenleistungsprüfung sein, um Informationen über die weiblichen Nachkommen eines Hengstes im Alter von drei-vier Jahren zu erlangen. Von Seiten des Zuchtverbandes sollten an dieser Stelle gezielte Fördermaßnahmen unternommen werden, um die röntgenologische Überprüfung der ersten Jahrgänge eines Hengstes zu ermöglichen. Zusätzlich sollte auch die Eigenverantwortung der Züchter gefördert werden. Durch Einbindung der Züchter in Selektionsentscheidungen würde das Bewusstsein für die Beachtung der gesundheitlichen Konstitution in der modernen Reitpferdezucht geweckt und gleichzeitig eine Eigenverantwortung aufgebaut, die bei den steigenden Ansprüchen des Marktes unerlässlich ist (MIESNER, 1996). 
Die Informationen der Nachkommen eines Hengstes stellen gleichzeitig eine wichtige Informationsquelle für die Zuchtwertschätzung der Osteochondrose der Halbbrüder der Nachkommen dar. Die röntgenologische Überprüfung aller Hengste (im hannoverschen Zuchtgebiet jährlich ca. 800 Hengste), die bei den der Körung vorgeschalteten Auswahlterminen vorgestellt werden, würde hier zu einem erheblichen Informationsgewinn beitragen. Auch die Überprüfung weiblicher Halbgeschwister eines Hengstes im Rahmen der Stutbuchaufnahme oder der Stutenleistungsprüfung würde eine weitere hilfreiche Informationsquelle darstellen. Zusätzliche Informationen könnten durch die Auswertung röntgenologischer Untersuchungen verwandter Auktionspferde erlangt werden. Hier sollte jedoch kein Einfluss auf das Vermarktungsgeschehen ausgeübt werden.

Die Durchführung der aufgezeigten Maßnahmen würde relativ rasch zu einer Anhäufung von Informationen führen, die durch die Verarbeitung im Rahmen der Zuchtwertschätzung zum frühzeitigen Vorliegen von Zuchtwerten für das Merkmal Osteochondrose bei wichtigen Selektionsentscheidungen, wie der Körung führen würden.

Abbildung 6 verdeutlicht die Ansatzpunkte für die Berücksichtigung der Osteochondrose anhand des folgenden Selektionsschemas:

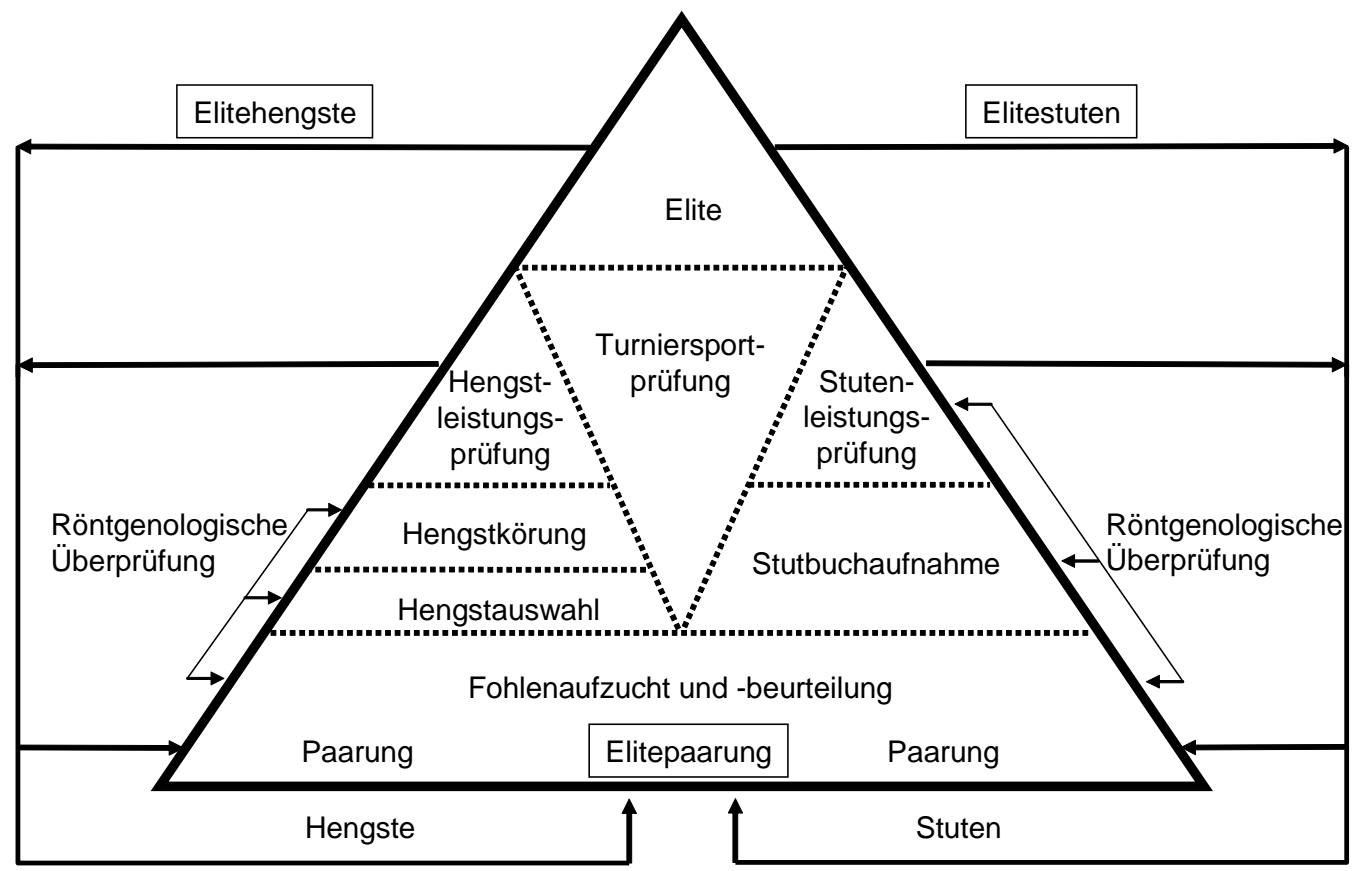

Abbildung 6: Ansatzpunkte für die röntgenologische Überprüfung auf OC innerhalb eines Zuchtprogramms 


\subsubsection{Bewertung weiterer simulierter Selektionsszenarien}

Die Diagnose der Osteochondrose erfolgt in der veterinärmedizinischen Praxis anhand röntgenologischer Untersuchungen. Röntgenuntersuchungen stellen neben der klinischen Untersuchung ein wesentliches Hilfsmittel bei der Diagnose von Gliedmaßenerkrankungen dar (WINTER, 1995). HOPPE (1983) verglich die anhand von Röntgenbilder geschätzte Größe von freien Gelenkskörpern mit der bei Operationen oder Autopsien tatsächlich festgestellten Größe der Fragmente und kam zu dem Schluss, dass die anhand von Röntgenbildern getroffenen Diagnosen als verlässlich angesehen werden können. Auch MERZ (1993) beurteilte anhand einer Reihenuntersuchung an den Zehen und Sprunggelenken deutscher Auktionspferde die Diagnose von isolierten Verschattungen im Röntgenbild als leicht durchführbar.

Andere Autoren sehen diese Diagnosemethode hingegen als nicht unproblematisch an. So vertritt BODENMÜLLER (1983) nach einer Analyse des Werts von Röntgenaufnahmen auf die Früherkennung der Podotrochlose aufgrund verschiedener Faktoren, welche die Aufnahmen beeinflussen können, sowie der Subjektivität der Beurteilung durch den Betrachter; eine kritische Einstellung gegenüber der Röntgendiagnose. Auch WINTER (1995) ermittelte anhand seiner Untersuchungen an den Röntgenbildern von 3566 Auktionspferden, die durch zwei Veterinärmediziner beurteilt wurden, einen signifikanten Einfluss des Beurteilers auf das Ergebnis. In der niederländischen Warmblutzucht wurde diesem Problem in der Form begegnet, dass die Röntgenbilder der Hengste bei der Körung nur in wenigen ausgewählten Tierarztpraxen angefertigt werden dürfen; die Beurteilung der Röntgenbilder erfolgt grundsätzlich durch dieselben Personen an der Universität von Utrecht.

Zusätzliche Informationen für die Durchführung eines effektiven Zuchtprogramms kann neben der Beurteilung der phänotypischen Leistungen auch der Einsatz molekulargenetischer Diagnostik liefern. So könnte die Diagnose der OC statt wie bisher anhand von Röntgenbildern, die den phänotypischen Leistungen eines Pferdes in diesem Merkmal entsprechen, durch eine Beurteilung des Genotyps erfolgen. Bei einem solchen Verfahren könnte man mögliche Fehlerquellen hinsichtlich der Röntgentechnik, der Darstellung und der Interpretation des Bewertenden ausschalten, und eine exaktere Diagnose treffen. 
Die Molekulargenetik erlaubt einen Einblick in das Genom eines Tieres; es können bestimmte Genombereiche mit einer Leistungsausprägung in Verbindung gebracht werden, sogenannte Quantitative Trait Loci (QTL). Eine Vorgehensweise, um die Diagnose einer Erkrankung anhand des Genotyps eines Individuums zu treffen, ist die Anwendung eines direkten Gentests für die zu untersuchende Erkrankung. Für einen direkten Gentest muss das Gen, das für die Ausprägung eines bestimmten Merkmals oder einer Erkrankung zuständig ist, identifiziert und seine Lokalisation auf dem Chromosomen bestimmt sein.

Diese Annahme wurde mit der Einbeziehung eines direkten Gentests für OC in die Selektion simuliert. Die Gewichtung für das Merkmal OC in Höhe von 0,2 liegt hier nicht wie bei allen anderen Selektionsmodellen auf dem Phänotyp des Merkmals OC, der die Selektion anhand des Röntgenbilds darstellt, sondern auf dem Genotyp des Merkmals OC. Um eine möglichst praxisnahe Anwendung des Gentests zu simulieren, fand eine Einbindung in die zweistufige Selektionsmethode statt. Auf der ersten Stufe findet wie bei den anderen Selektionsmodellen die Selektion aufgrund der Leistungen in den Merkmalen Dressur und Springen statt. Auf der zweiten Stufe werden die in den Leistungsmerkmalen überragenden Pferde der Überprüfung ihres Genotyps mittels eines direkten Gentest unterzogen. Die erzielten Ergebnisse werden im Vergleich zu den Selektionserfolgen dargestellt, die mit der Selektion aufgrund von Röntgenbildern erzielt werden.

Tabelle 36: OC-Frequenzen bei Anwendung/Nichtanwendung eines OC-Gentests (Modell mit vier Klassen, zweistufige Selektion)

\begin{tabular}{|cc|cc|}
\hline \multicolumn{2}{|c|}{ Remontierungsrate $^{\mathbf{1})}$} & \multicolumn{2}{c|}{ OC-Frequenz $^{2)}$} \\
Erste Stufe & Zweite Stufe & 4- Klassen $^{\text {Modell }}{ }^{3)}$ & $\begin{array}{c}\text { 4- Klassen Modell } \\
\text { mit OC-Gentest }\end{array}$ \\
\hline 0,01 & 1 & 0,205 & 0,205 \\
\hline 0,02 & 0,5 & 0,145 & 0,124 \\
\hline 0,05 & 0,2 & 0,138 & 0,106 \\
\hline 0,1 & 0,1 & 0,138 & 0,106 \\
\hline
\end{tabular}

2) Durchschnittsfrequenz für OC in der ersten Generation $=0,2 \quad 3$ ) mittlerer Standardfehler $=0,001$

4) mittlerer Standardfehler $=0,001$ 
Die OC-Frequenz sinkt auf einen Wert von 0,106 in der fünften Generation bei Anwendung einer Remontierungsrate von 0,05 auf der ersten und 0,2 auf der zweiten Selektionsstufe. Im Vergleich dazu wird bei Anwendung des Modells mit vier Klassen ohne Gentest und gleicher Remontierungsraten ein Wert von 0,138 erreicht. Somit kann die Aussage getroffen werden, dass die Selektion anhand eines direkten Gentests zu größeren Selektionserfolgen hinsichtlich der Reduktion der OCFrequenz führen würde, als die herkömmliche Selektion, die auf der Diagnose anhand von Röntgenbildern beruht. Dieser Fortschritt bei der Reduktion der OCFrequenz ist darauf zurückzuführen, dass bei dem Modell mit OC-Gentest die Annahme erfolgt, dass das Gen, welches für die Entstehung und Ausprägung der Osteochondrose verantwortlich ist, charakterisiert und lokalisiert wurde. Anhand dieses direkten Gentests könnten also befallene Pferde eindeutig diagnostiziert und selektiert werden, was zu einem großen Zuchtfortschritt bei der Reduzierung der OCFrequenz führen würde. Somit sind die Ergebnisse, die das Vorhandensein eines Gentests simulieren, vorsichtig zu bewerten, da sie auf Annahmen beruhen, die noch nicht belegt werden konnten, denn bisher sind die Gene, die eine Rolle für die Entstehung von OC beim Pferd spielen, noch nicht charakterisiert (LÖHRING, 2004). Lediglich in der Schweinezucht wurde von der Entdeckung von Markern für das Beinschwächesyndrom beim Schwein berichtet (LEE et al., 2003).

Da derzeit kein direkter Gentest für die Diagnose der Erkrankung OC vorhanden ist, werden Marker verwendet, um Wahrscheinlichkeitsaussagen über das Vorhandensein bestimmter Allele zu treffen. Marker können als Markierung oder Hinweis auf phänotypischer oder genotypischer Ebene angesehen werden, die auf das Vorhandensein eines Gens hindeuten können. Mit Hilfe von Mikrosatellitenmarkern identifizierten LÖHRING $(2002,2003)$ und BÖDECKER et al. (2004) Genombereiche die QTL für OC beim Pferd enthalten, so dass diese Informationen zur Verfeinerung herkömmlicher Zuchtmethoden in einem ausgereiften Zuchtprogramm künftig Berücksichtigung finden könnten. Auch KOENEN et al. (2000) und PIERAMATI et al. (2003) sehen mit Hilfe der Markergestützten Selektion Möglichkeiten, die Frequenz osteochondrotischer Veränderungen in Populationen zu verringern. LOOFT (2004) sieht bei der Anwendung der Genomanalyse große Chancen für die Züchtung gesunder Pferde, führt aber auch den hohen logistischen Aufwand und entsprechende Kosten ins Feld. 
$\mathrm{Da}$ in der Pferdezucht nur in geringem Umfang Informationen zum Gesundheitsstatus der Population zur Verfügung stehen, bietet die Genomanalyse hier wichtige Ansatzpunkte. Durch die systematische Erfassung von Erbfehlern und dem Auftreten von Krankheiten, und der Speicherung in Datenbänken, könnte die Grundlage geschaffen werden, um Methoden der Genomanalyse auch in der Pferdezucht in größerem Umfang zu nutzen. Auch KALM (2002) weist auf die Notwendigkeit einer Dokumentation von Gesundheitsparametern hin. Seiner Meinung nach könnten Konzepte zur Verbesserung der Gesundheitssituation ohne Datenerfassung und Dokumentation nicht entwickelt werden. Einen Ansatz zur Erfassung sieht er in einer engen Zusammenarbeit zwischen Tierarzt und Tierhalter, wobei eine zeitnahe Erfassung unerlässlich wäre. Die verschiedenen Befunde sollten nach einem definierten Schlüssel dokumentiert werden, wobei er den Gliedmaßenerkrankungen eine besonders hohe Priorität zuordnet. Weitere Komplexe sollten entsprechend der Häufigkeit und wirtschaftlichen Bedeutung aufgenommen werden. Die Zuchtorganisationen sollten seiner Meinung nach in Zusammenarbeit mit Tierärzten ein Erfassungskonzept erarbeiten, bei der Umsetzung in der Praxis könnten die dokumentierten Erfahrungen aus der Rinderzucht genutzt werden.

Nicht nur die Einbeziehung molekulargenetischer Methoden würde zu einem Selektionsgewinn bei der Reduzierung der OC-Frequenz beitragen, auch die zusätzliche Einbeziehung der Stuten in die Selektion könnte einen wichtigen Beitrag leisten. Da die Anzahl der Nachkommen, die ein Hengst produzieren kann, um ein vielfaches höher ist als die Nachkommenanzahl, die von Stuten produziert werden kann, ist der Einfluss von Hengsten auf züchterische Entwicklungen höher zu bewerten als der, der von der Stutenseite ausgeht. Deshalb konzentrieren sich die züchterischen Maßnahmen in traditionellen Zuchtprogrammen der Warmblutzucht vor allem auf die Hengste. In der Pferdezucht sind die Selektionsintensitäten bei Stuten gegenüber weiblichem Zuchtmaterial anderer landwirtschaftlicher Nutztiere niedriger (MIESNER, 1996). Nach SCHWARK (1988) machen die Stutenpfade einen Anteil von etwa einem Drittel am Gesamtzuchtfortschritt aus. Um dieses Potential nutzbar zu machen, sollten die Auswirkungen bei Einbeziehung von Stuten in Selektionsentscheidungen genauer untersucht werden. 
KOENEN et al. (2000) simulierte das Szenario einer zusätzlichen Einbeziehung der Stuten in die Selektion gegen OC und kam zu dem Ergebnis, dass sich mit dieser Maßnahme die OC-Frequenz von 25\% auf 13\% reduzieren ließe, was einer Abnahme in Höhe von 2,8\% pro Generation entspräche. Auch in der Studie von PIERAMATI et al. (2003) wurde dieses Szenario simuliert, bei einer Ausgangsfrequenz von 16\% wurde eine Reduktion der OC-Frequenz auf 1,8\% innerhalb fünf Generationen erzielt.

Ein Vergleich der unterschiedlichen Ergebnisse, die bei Einbeziehung der Stuten erzielt werden könnte, mit den Ergebnissen der Selektion, die lediglich die Hengste einbezieht, fand auch innerhalb dieser Studie statt.

Tabelle 37: OC-Frequenzen bei Selektion Hengste und Stuten vs. Selektion nur der Hengste (genetisches Model I, einstufige Selektion)

\begin{tabular}{|c|c|c|c|c|}
\hline \multicolumn{3}{|c|}{ Selektionsgewichte auf } & \multicolumn{2}{|c|}{ OC-Frequenz $^{1)}$} \\
\hline Dressur & Springen & OC & Stuten+Hengste ${ }^{2)}$ & nur Hengste $^{3 \text { ) }}$ \\
\hline 0,4 & 0,4 & 0,2 & 0,087 & 0,132 \\
\hline 0,4 & 0,4 & 0,4 & 0,056 & 0,078 \\
\hline 0,4 & 0,4 & 0,8 & 0,040 & 0,070 \\
\hline
\end{tabular}

Bei der Bewertung der Ergebnisse sollte berücksichtigt werden, dass hier das genetische Modell I im Rahmen einer einstufigen Selektion verwendet wurde, das heißt, dass bei dieser Selektion alle einbezogenen Pferde einer Untersuchung hinsichtlich ihres röntgenologischen Status unterzogen wurden, und somit eine höhere Reduktion der OC-Frequenz zu erwarten ist, als bei der Anwendung der zweistufigen Selektion. Die Ergebnisse verdeutlichen jedoch, dass bei der Einbeziehung der Stuten in die Selektion Potential für die weitere Reduktion der OCFrequenz vorhanden ist. Unter Berücksichtigung des finanziellen Aufwands könnte dieses Potential durch röntgenologische Überprüfungen im Rahmen verschiedener Selektionsstufen, denen Zuchtstuten unterzogen werden, wie der Stutbuchaufnahme oder der Stutenleistungsprüfung, genutzt werden, um zum Fortschritt bei der Reduzierung der OC-Frequenz innerhalb der Population beizutragen. 
Die Durchführung von röntgenologischen Überprüfungen im Rahmen der genannten Ansatzpunkte wurde in dieser Untersuchung bereits für die röntgenologische Überprüfung der Nachkommen und Halbgeschwister eines Hengstes anlässlich der Zuchtwertschätzung vorgeschlagen, und würde somit auch bei einer Integration der Stutenseite in die Selektion gegen OC zu einer effizienteren Selektionsstrategie gegen diese Erkrankung beitragen.

Die Ergebnisse dieser Untersuchung verdeutlichen, dass die Merkmalskomplexe Leistung und Gesundheit nicht getrennt voneinander betrachtet werden sollten, da der einwandfreie Gesundheitsstatus eines Pferdes die Vorraussetzung für Leistungen in den Merkmalen Dressur und Springen ist. Zusätzlich wird die Integration funktionaler Merkmale in Zuchtprogramme aufgrund steigender Ansprüche hinsichtlich Gesundheit und Langlebigkeit von Warmblutpferden immer bedeutsamer. Aus diesen Gründen verdienen sowohl Leistungs- als auch Gesundheitsmerkmale ihre Berücksichtigung im Zuchtziel und sollten unter Berücksichtigung ihres züchterischen und ökonomischen Nutzens in Selektionsentscheidungen eingebunden werden. 


\subsection{Schlussfolgerungen/Empfehlungen}

Die Ergebnisse der vorliegenden Arbeit machen deutlich, dass durch eine Integration der Selektion gegen Osteochondrose große Erfolge bei der Reduzierung der OCFrequenz erzielt werden können. Anhand der Ergebnisse konnten folgende Erkenntnisse gewonnen werden:

- hinsichtlich der genetischen Modelle scheint durch das genetische Modell II, bei dem OC als normalverteiltes Merkmal beschrieben wird, eine größere Annäherung an das tatsächliche genetische Modell der Erkrankung Osteochondrose gelungen $\mathrm{zu}$ sein

- eine hinsichtlich ihres OC-Status komplett durchgeröntgte Population, wie sie bei der einstufigen Selektion simuliert wird, führt $\mathrm{zu}$ einer größeren Reduktion der OC-Frequenz, ist aber in finanzieller Hinsicht schwer erreichbar

- die zweistufige Selektion führt im Vergleich zu einer geringeren Reduktion der OC-Frequenz, ist aber in finanzieller Hinsicht ein Gewinn und stellt somit eine praxisnähere Simulation der Selektion in der Pferdezucht dar

- bezüglich der optimalen Gewichtung der Merkmale Dressur, Springen und OC innerhalb des Selektionsindexes hat sich eine Gewichtung, die den Heritabilitäten der Merkmale entspricht, als optimal herausgestellt

- die Fortschritte bei der OC- Reduktion, die durch die Anwendung der Remontierungsraten innerhalb der zweistufigen Selektion erzielt werden, sind abhängig von der relativen ökonomischen Gewichtung des Merkmals OC

Hinsichtlich der entwickelten Selektionsmodelle können folgende Empfehlungen ausgesprochen werden, um eine optimale Integration der Selektion gegen OC in Selektionsentscheidungen zu gewährleisten:

- im Vergleich der drei simulierten Auswahlverfahren bei der Körung führt das Modell mit Zuchtwertschätzung zu den größten Erfolgen bei der Reduzierung der OC-Frequenz, gefolgt von dem Modell mit vier Röntgenklassen und dem Ausschlussmodell 
- das Selektionsmodell mit Zuchtwertschätzung führte auch hinsichtlich der Zuchtwerte der Leistungsmerkmale Dressur und Springen zu den größten Fortschritten, das Modell mit vier Klassen lag mit seinen Fortschritten dahinter und das Ausschlussmodell führte zu den geringsten Fortschritten bei den Leistungsmerkmalen

- aufgrund der ermittelten Ergebnisse wird das Modell mit Zuchtwertschätzung im Vergleich der anderen Selektionsmodelle präferiert und als geeignetes Selektionsverfahren für die Implementierung der Selektion gegen OC in Zuchtprogramme empfohlen

- die Durchführung einer Zuchtwertschätzung für das Merkmal Osteochondrose würde $\mathrm{zu}$ weiteren Sicherheiten bei Selektionsentscheidungen beitragen, da bereits frühzeitig Zuchtwerte für Dressur, Springen und OC vorlägen

- die Informationen für die Zuchtwertschätzung sollten anhand der röntgenologischen Überprüfung von verwandten Pferden gewonnen werden, röntgenologische Überprüfungen sollten deshalb in Selektionsabläufe wie die Stutenleistungsprüfung oder die Hengstvorauswahl eingebunden werden

- die Simulation eines Gentests für OC und die verstärkte Einbeziehung der Stuten in die Selektion gegen OC offenbaren ein großes Potential für eine effizientere Selektion gegen OC

- eine Erfassung und Einordnung des tatsächlichen wirtschaftlichen Verlustes ausgehend von der Osteochondrose ist notwendig, um die Implementierung der Selektion gegen $\mathrm{OC}$ in Zuchtprogramme auch im Hinblick auf die ökonomischen Auswirkungen richtig bewerten zu können

- im Hinblick auf den multifaktoriellen Hintergrund der Osteochondrose sollte eine Optimierung der Aufzucht- und Haltungsbedingungen angestrebt werden 


\section{Zusammenfassung}

Im Rahmen der vorliegenden Arbeit wurden die Auswirkungen von verschiedenen Selektionsstrategien gegen die Gelenkserkrankung Osteochondrose auf eine simulierte Warmblutpopulation untersucht. Die simulierte Population wurde in Anlehnung an Strukturverhältnisse aus der Pferdezucht erstellt, um eine Übertragbarkeit der erzielten Ergebnisse auf reale Populationen zu gewährleisten. Die Selektion wurde anhand eines Selektionsindexes vollzogen, der die Merkmale Dressur, Springen und Osteochondrose (OC) umfasst. Die Gewichtung der Merkmale innerhalb des Selektionsindexes variierte. Auf der einen Seite erfolgte die Beobachtung der Veränderungen der OC-Frequenz ausgehend von einer Frequenz von 0,2 in der Basisgeneration über fünf Generationen und 50 Wiederholungen. Ebenso wurden die Einflüsse verschiedener Selektionsmethoden auf die Zuchtwerte der Leistungsmerkmale Dressur und Springen erfasst.

Zwei verschiedene genetische Modelle wurden entwickelt, die eine Annahme über das mögliche Vererbungsmodell der OC treffen. Innerhalb des ersten Modells wird als OC ein Genort mit zwei Allelen beschrieben, in dem zweiten Modell ist OC ein normalverteiltes Merkmal. Das genetische Modell I führte mit einer Reduzierung auf einen Wert von 0,087 in der fünften Generation zu größeren Selektionserfolgen hinsichtlich der Reduktion der OC-Frequenz, als das genetische Modell II mit einem Wert von 0,171 innerhalb der einstufigen Selektion. Aufgrund der realistischeren Annahme über die Vererbung von OC wurde das genetische Modell II aber als wahrscheinlicher angenommen und als Basismodell für weitere entwickelte Selektionsmodelle herangezogen.

Die Selektion vollzieht sich durch zwei unterschiedliche Selektionsmethoden, die ein- und die zweistufige Methode. Die einstufige Strategie beinhaltet die Selektion der drei Merkmale innerhalb einer Selektionsstufe. Bei der zweistufigen Selektion findet auf der ersten Stufe eine Selektion auf die Dressur- und Springeignung statt, auf der zweiten Stufe werden Pferde mit überdurchschnittlichen Leistungen in diesen Merkmalen einer röntgenologischen Überprüfung ihres OC-Status unterzogen. Bei dieser Methode werden nur die Hengste in die Selektion einbezogen. 
Die Selektionsschärfe wird über die Angabe von Remontierungsraten auf der ersten Selektionsstufe geregelt. Bei Anwendung der einstufigen Selektionsmethode, die eine röntgenologische Untersuchung der gesamten Population hinsichtlich des OCStatus vorsieht, wurde mit einem Wert von 0,087 eine höhere Reduktion der OCFrequenz verzeichnet. Die zweistufige Selektionsmethode führte zu einer geringeren Reduktion der OC-Frequenz (0,120), stellt im Vergleich zur einstufigen Selektion jedoch eine finanzielle Erleichterung dar, da hier nur die Pferde mit überragenden Leistungen in Dressur und Springen röntgenologisch auf OC überprüft werden. Im Hinblick auf die bessere Umsetzbarkeit wurde das zweistufige Selektionsmodell präferiert und in weitere entwickelte Selektionsszenarien einbezogen.

Weiterhin wurden verschiedene Selektionsszenarien entwickelt, welche die Auswahlmethoden bei der Hengstkörung simulieren. Auf Basis des genetischen Modells II entstand ein Modell, das den Phänotyp in vier Klassen einteilt, die den vier Röntgenbewertungsklassen nachempfunden sind. Auf der Basis dieses Modells wurde ein Ausschlussselektionsmodell entwickelt, bei dem Hengste, deren röntgenologische Befunde in die Röntgenklassen III oder IV eingeteilt wurden, von weiteren Selektionsentscheidungen ausgeschlossen werden. Das Szenario einer Zuchtwertschätzung für OC wurde innerhalb eines weiteren Selektionsmodells simuliert. Hinsichtlich der Reduzierung der OC-Frequenz führte das Modell, das die Anwendung einer Zuchtwertschätzung vorsieht, zu den größten Erfolgen $(0,124)$, gefolgt von dem Modell mit vier Röntgenklassen $(0,138)$ und dem Ausschlussmodell mit der geringsten Reduktion der OC-Frequenz $(0,142)$. Auch bei den Zuchtwerten der Leistungsmerkmale Dressur und Springen führte das Modell mit Zuchtwertschätzung zu den größten Fortschritten. Das Selektionsmodell mit vier Röntgenklassen lag mit seinen Ergebnissen dahinter und das Ausschlussmodell führte zu den geringsten Fortschritten bei den Zuchtwerten der Leistungsmerkmale.

Weitere Selektionsszenarien wie das Vorhandensein eines Gentests für OC oder die verstärkte Einbeziehung der Stuten in die Selektion gegen OC wurden simuliert. Beide Szenarien offenbarten ein großes Potential für eine effizientere Selektion gegen OC. 
Anhand der Ergebnisse wird deutlich, dass die Osteochondrose-Frequenz einer Population durch gezielte züchterische Maßnahmen innerhalb eines Zuchtprogramms gesenkt werden kann. Im Vergleich der simulierten Selektionsszenarien wird aufgrund der erzielten Ergebnisse das Modell mit Zuchtwertschätzung präferiert und für die Anwendung innerhalb eines Zuchtprogramms empfohlen. 


\section{Summary}

In the course of the present study, effects of selection strategies to prevent the joint disease osteochondrosis on a simulated horse population have been evaluated. The simulated population was developed following the conditions of a real horse population to ensure realistic results. The selection takes place within a selection index, which considers the three traits dressage, jumping and ostechondrosis (OC). The selection weights put on the traits differed. On the one hand the changes of the OC-frequency proceeding on a basic frequency of 0.2 have been observed over 5 generations and 50 repetitions. The influences of different selection strategies on the breeding values of the performance traits have been recorded as well.

Due to the fact that the transmission of OC is unknown, two different genetic models for the definition of osteochondrosis have been developed. Model I describes OC as a one-gene locus trait with two alleles, in model II OC is simulated as a normally distributed trait. The results showed that the frequency of OC decreased more by using genetic model I (0.087) compared to model II (0.171). Because of the more realistic assumption of the possible transmission of OC, model II was considered as more likely and used for the further development of other genetic models.

Two different selection strategies were applied for both different genetic models. The one-step selection model selects horses for the three different traits within one step. In this strategy all horses are X-rayed for OC. The two-step selection model selects horses based on their performance in dressage and jumping at the first step; at the second step horses are selected on both the performance in dressage and jumping and on OC. Only the superior horses in dressage and jumping are X-rayed for OC. This procedure resembles the selection at the stallion approval, so it considers only the stallions of the simulated population for the selection against OC. The selection intensity at the two steps can be adjusted by selection rates. The one-step selection model led to a higher reduction of the OC frequency (0.087). Due to the fact, that in this strategy the complete population is x-rayed for OC, the realization seems to be difficult because of the necessary high expenses. 
In the two-step selection model the selection against OC takes place at the second step, after horses already have been selected for their performance in dressage and jumping, so that this selection model led to lower progress in the reduction of the OC-frequency $(0.120)$ but also to lower costs for radiological observations. In view of an easier realization, the two-step selection model was included in further selection models.

On the basis of the presented genetic model II and the two-step selection strategy, several selection scenarios were developed, which simulates the selection at the stallion approval. The genetic model II with two phenotypic classes was refined to divide the phenotype into the 4 common x-ray classes. As a further development of this 4-class model, the exclusion of stallions with an x-ray class III and/or IV from selection was simulated. Besides, a selection against OC based on estimated breeding values of OC and performance traits was developed on the basis of the 4-class model. The results showed, that the frequency of OC decreased most by using the model, which calculates breeding values for OC $(0.124)$, followed by the 4-class model $(0.138)$ and the model with exclusion of stallions with the lowest reduction of the OC-frequency $(0.142)$. Concerning the breeding values of the performance traits dressage and jumping, the model with breeding values led again to the highest progress, followed by the 4-class model and the model with exclusion showed the lowest progress in the performance traits.

Further selection scenarios have been developed, like the selection with an OC-genetest or the consideration of mares in the selection against OC. Both scenarios showed great potential for a more efficient selection against OC.

In view of the results gained in this study the conclusion can be drawn, that the OCfrequency could be reduced by specific selection strategies in a breeding program. By comparing the different selection scenarios due to the results, the model with breeding values is preferred and recommended for the use in breeding programs. 


\section{Tabellen- und Abbildungsverzeichnis}

Tabelle 1 Abgangsursachen und deren Häufigkeiten bei Sportpferden

Tabelle 2 Einteilungsschema der erhobenen Röntgenbefunde nach der europäischen Röntgenkommission (1993)

Tabelle 3 Überblick über bisherige populationsgenetische Studien zur Osteochondrose

Tabelle 4 Überblick über die Ergebnisse der Studien von WILLMS (1995) 38 und WINTER (1998)

Tabelle 5 Überblick über entwickelte genetische Modelle und Selektionsmethoden

Tabelle 6 Verwendete Selektionsgewichte in dieser Untersuchung

Tabelle 7 Verwendete Remontierungsraten für die Selektion der Hengste innerhalb der zweistufigen Selektionsmethode

Tabelle 8 OC-Frequenzen bei Anwendung der genetischen Modelle I und II innerhalb der einstufigen Selektionsmethode 
Tabelle 9 OC-Frequenzen bei Anwendung der genetischen Modelle I und II innerhalb der zweistufigen Selektionsmethode

Tabelle 10 Veränderungen der Zuchtwerte für Dressur und Springen bei Anwendung unterschiedlicher Selektionsgewichte gegen OC (einstufige Selektion, genetisches Modell I)

Tabelle 11 Mittelwerte der OC-Frequenzen und der Zuchtwerte der Leistungsmerkmale bei Anwendung der zweistufigen Selektion mit unterschiedlichen Remontierungsraten (genetisches Modell II mit zwei Klassen)

Tabelle 12 OC-Frequenzen bei Darstellung der OC in zwei- bzw. vier phänotypischen Klassen

Tabelle 13 OC-Frequenzen bei Anwendung der Ausschlussselektionsmodelle

Tabelle 14 Mittlere Zuchtwerte der Leistungsmerkmale bei Anwendung der Ausschlussselektionsmodelle

Tabelle 15 OC-Frequenzen bei Anwendung des Modells mit vier Klassen ohne Ausschluss und der Anwendung der Ausschlussselektionen mit Ausschluss der Röntgenklasse IV/Ausschluss der Röntgenklassen III und IV 
Tabelle 16 Mittlere Zuchtwerte der Leistungsmerkmale bei Anwendung des Modells mit vier Klassen ohne Ausschluss und der Anwendung der Ausschlussselektionen mit Ausschluss der Röntgenklasse IV/Ausschluss der Röntgenklassen III und IV

Tabelle 17 OC-Frequenzen bei Selektion der Hengste nach geschätzten Zuchtwerten

Tabelle 18 Mittlere Zuchtwerte der Leistungsmerkmale bei Selektion der Hengste nach geschätzten Zuchtwerten

Tabelle 19 OC-Frequenzen bei Anwendung verschiedener Selektionsverfahren 91 für Hengste

Tabelle 20 Mittlere Zuchtwerte der Leistungsmerkmale bei Anwendung verschiedener Selektionsverfahren für Hengste

Tabelle 21 OC-Frequenzen bei Anwendung/Nichtanwendung eines OC-Gentests (Modell mit vier Klassen, zweistufige Selektion)

Tabelle 22 OC-Frequenzen bei Selektion Hengste+ Stuten vs. Selektion nur der Hengste (genetisches Model I, einstufige Selektion)

Tabelle 23 OC-Frequenzen bei Anwendung der genetischen Modelle I und II innerhalb der einstufigen Selektionsmethode 
Tabelle 24 OC-Frequenzen bei Anwendung der genetischen Modelle I und II innerhalb der zweistufigen Selektionsmethode

Tabelle 25 Veränderungen der Zuchtwerte für Dressur und Springen bei Anwendung unterschiedlicher Selektionsgewichte gegen OC (einstufige Selektion, genetisches Modell I)

Tabelle 26 Mittelwerte der OC-Frequenzen und der Zuchtwerte der Leistungsmerkmale bei Anwendung der zweistufigen Selektion mit unterschiedlichen Remontierungsraten (genetisches Modell II mit zwei Klassen)

Tabelle 27 OC-Frequenzen bei Darstellung der OC in zwei bzw. vier phänotypischen Klassen

Tabelle 28 OC-Frequenzen bei Anwendung der Ausschlussselektionsmodelle

Tabelle 29 Mittlere Zuchtwerte der Leistungsmerkmale bei Anwendung der Ausschlussselektionsmodelle

Tabelle 30 OC-Frequenzen bei Anwendung des Modells mit vier Klassen ohne Ausschluss und der Anwendung der Ausschlussselektionen mit Ausschluss der Röntgenklasse IV/ Ausschluss der Röntgenklassen III und IV 
Tabelle 31 Mittlere Zuchtwerte der Leistungsmerkmale bei Anwendung des Modells mit vier Klassen ohne Ausschluss und der Anwendung der Ausschlussselektionen mit Ausschluss der Röntgenklasse IV/Ausschluss der Röntgenklassen III und IV

Tabelle 32 OC-Frequenzen bei Anwendung verschiedener Selektionsverfahren für Hengste

Tabelle 33 Mittlere Zuchtwerte der Leistungsmerkmale bei Anwendung verschiedener Selektionsverfahren für Hengste

Tabelle 34 Gesamtzuchtfortschritte der Selektionsmodelle bei unterschiedlicher ökonomischer Gewichtung der Einzelmerkmale

Tabelle 35 Ergebnisse vergleichbarer Simulationsstudien zur züchterischen Bekämpfung der Osteochondrose

Tabelle 36 OC-Frequenzen bei Anwendung/Nichtanwendung eines OC-Gentests (Modell mit vier Klassen, zweistufige Selektion)

Tabelle 37 OC-Frequenzen bei Selektion Hengste und Stuten vs. Selektion nur der Hengste (genetisches Model I, einstufige Selektion) 
Abbildung 1 Einflussfaktoren auf die Entstehung der Osteochondrose (modifiziert nach JEFFCOTT, 2004)

Abbildung 2 Aktuelles Hannoversches Zuchtprogramm

(Quelle: Der Hannoveraner, Ein Leitfaden für den Züchter)

Abbildung 3 Einteilung des normalverteilten Phänotyps in vier Klassen bei genetischem Modell II mit vier Klassen

Abbildung 4 Veränderung der OC-Frequenzen über fünf Generationen (Selektionsgewichte $0,4 / 0,4 / 0,2$ )

Abbildung 5 Veränderungen der Zuchtwerte für Dressur und Springen bei Anwendung verschiedener Selektionsgewichte

Abbildung 6 Ansatzpunkte für die röntgenologische Überprüfung auf OC innerhalb eines Zuchtprogramms 


\section{Literaturverzeichnis}

ANDERSON-EKLUND, L., UHLHORN, H., LUNDHEIM, N., DALIN, G., ANDERSSON, L. (2000):

Mapping quantitative Trait Loci for principal components of bone measurements and osteochondral scores in a wild boar $\mathrm{x}$ large white

Genetical Research 75: 223-230

AHTILA, L., SAASTAMOINEN, M.T. (2004):

Effect of nutrition on the growth curve of weanling foals

In: EAAP Publication No. 114, The growing horse: nutrition and prevention of growth disorders, Wageningen Academic Publishers, 2004

ARNAN, P. (2004):

Röntgenologische Befunderhebung bei zweijährigen Warmblütern

Dissertation, Freie Universität Berlin, Fakultät für Veterinärmedizin

ARNASSON, T. (1983):

Prediction of breeding values in horses by the BLUP-method

34. Jahrestagung der EVT, Madrid

BAKER, J.R., ELLIS, C.E. (1981):

A survey of post mortem findings in 480 horses 1958 to 1980: (2) disease processes not directly related to the cause of death

Equine Vet. J. 13, 47-50

BADE, B (1974):

Schätzung genetischer Parameter für Leistungsmerkmale Hannoverscher Reitpferde

Dissertation, Universität Göttingen, Fakultät für Agrarwissenschaften

BARNEVELD, A., P.R. VAN WEEREN (1999a):

Early changes in the distal intertarsal joint of Dutch warmblood foals and the influence of exercise on bone density in the third tarsal bone.

Equine Vet. J. Suppl. $\underline{31}$, 16-25

BARNEVELD, A., P.R. VAN WEEREN (1999b):

The effect of exercise on the distribution and manifestation of osteochondrotic lesions in the warmblood foal

Equine Vet. J. Suppl. $\underline{31}$, 67-73

BATHE, A.P. (1994):

245 fractures in thoroughbred racehorses: results of a 2 years prospective study in Newmarket, Proceedings of the American Association of Equine practioneers $\underline{40}, 275-276$ 
BEARD, W.L., BRAMLAGE, L.M., SCHNEIDER, R.K., Embertson, R.M. (1994): Postoperative racing performance in standardbreds and thoroughbreds with osteochondrosis of the tarsocrural joint: 109 cases (1984-1990)

JAVMA, Vol 204, 10, 1655-1659

BILLINGHURST, R.C., BRAMA, P.A., van WEEREN P.R., KNOWLTON M.S., MCILWRAITH C.W. (2004)

Evaluation of serum concentrations of biomarkers of skeletal metabolism and results of radiography as indicators of severity of osteochondrosis in foals. Am J. Vet. Res. 2004 65 (2): 143-50.

BINNS, M., SWINBURNE, J.E., BREEN, M. (2000):

Molecular genetics of the horse

in: Genetics of the horse

CAB Int'1., 109-121

BÖHM, D., Nagel, M.L (1980):

Beitrag zur Röntgendiagnostik der Gelenkserkrankungen bei jungen Pferden.

Berl. Münch. Tierärztl. Wochenschrift 93, 161-163

BÖNEKER, C., MÜLLER, D., WITTWER, C., KUIPER, H., DRÖGEMÜLLER, C., DISTL, O. (2004):

Physikalische Kartierung von Pferdechromosomen und vergleichende Genomanalyse mit syntänischen Genomabschnitten anderer Genomabschnitten anderer Säugerspezies

Vortragstagung der DGfZ und GfT, Rostock

BORCHERS, A. (2002):

Die Körpergewichts- und Körpergrößenentwicklung des Warmblutfohlens während des ersten Lebenshalbjahres in Bezug zur Energie- und Proteinzufuhr sowie zum Auftreten der Osteochondrose.

Dissertation, Tierärztliche Hochschule Hannover

BRAMA, P.A., TeKoppele, J.M., Bank, R.A., Barneveld, A., van Weeren, P.R. (2002):

Development of biochemical heterogenity of articular cartilage:

influences of age and exercise.

Equine Vet. J. 34, 265-269

BRAMLAGE, L.R. (1986):

Pathogenesis of developmental orthopaedic problems in: Developmental

Orthopaedic Disease Symposium

Ed: C.W. MC ILWRAITH, AQHA, Amarillo, Texas

BREHM, W., STAECKER, W. (2000):

Osteochondrosis in the tarsocrural joint of Standardbred trotters Correlation between radiographic findings and racing performance Pferdeheilkunde 16, 590-593 
BRIDGES, C.H., HARRIS, E.D. (1988):

Experimentally induced cartilaginous fractures (osteochondritis dissecans) in foals fed low copper diet

J. Am. Vet. Med. Assoc., 193, 215-221

BROCKMANN, A. (1998):

Entwicklung einer Eigenleistungsprüfung im Feld für Hengste unter

Berücksichtigung der Turniersportprüfung

Dissertation, Universität Göttingen, Fakultät für Agrarwissenschaften

BRUNS, E. (1987):

Vergleich verschiedener BLUP-Zuchtwertschätzmodelle in der

Milchrinderzucht mit Hilfe der Computersimulation

Habil. Schrift, Göttingen, Fakultät für Agrarwissenschaften

BRUNS, E., GLODEK, P. (1976):

Erfahrungen mit simulierten Selektionsexperimenten in der Lehre

$26^{\text {th }}$ Annual Meeting of the EAAP, Zürich

BRUNS, E. (2001):

Berücksichtigung von Merkmalen der Gesundheit in der Züchtung von

Sportpferden

Arch. Tierz., Dummerstorf, 44 (2), 119-127

BRUNS, E. (2005):

Mögliche Auswirkungen des Embryotransfers auf Zuchtprogramme

Tagungsband Pferde-Workshop Uelzen, 2005

BUTLER, v. I., ARMBRUSTER, B. (1984):

Struktur und Abgangsursachen bei Schlachtpferde

Dt. tierärztl. Wschr. 91, 330-331

CAETANO, A.R., SHIUE, Y.L., LYONS, L.A., O'BRIEN, S.J., LAUGHLIN, T.F., BOWLING, A.T., MURRY, J.D. (1999):

A comperative gene map of the horse (Equus caballos)

Genome research $\underline{9}, 1239-1249$

CAMARDA, A.J., BUTLER, W.T., FINKELMANN, R.D., NANCI, A. (1987):

Immunocytochemical localization of gamma-carboxyglutamic acid-containing proteins (osteocalcin) in rat bone and dentin

Calcif Tissue Int., $\underline{40}, 349-355$

CARLSTEN, J., SANDGREN, B., DALIN, G. (1993):

Development of osteochondrosis in the tarsocrural joint and osteochondral fragments in the fetlock joint of Standardbred trotters. I. A. radiological survey Equine Vet. J. Suppl. 16, 42-47

CLAUSEN, M., Preisinger, R., Kalm, E. (1990):

Analyse von Krankheitsdaten in der deutschen Warmblutzucht.

Züchtungskunde $\underline{62}, 167-178$. 
CHRISTMANN, L. (2004):

Neue röntgenologische Kriterien

In: Der Hannoveraner 8/04

CHRISTMANN; L. (2005):

Zur Berücksichtigung von röntgenologischen Befunden

in der Arbeit der Zuchtverbände

4. Pferdeworkshop, Uelzen 2005

COLLINDER, E., RASMUSON, M. (2000):

Genetic aspects of disease in horses

In: The genetics of the horse

CAB Int'l., 157-170

CORNELISSEN, B. P., VAN WEEREN, P.R., EDERVEEN, A.G., BARNEVELD,

A. (1999):

Influence of exercise on bone mineral density of immature cortical

trabecular bone of the equine metacarpus and proximal sesamoid bone

Equine Vet. J. Suppl. $\underline{31}, 79-85$

CYMBALUK, N.F. (1990):

Cold housing effects on growth and nutrient demand of young horses.

J. Anim. Sci. $\underline{68}, 3152-3162$.

CYMBALEK, N.F., CHRISTISON, G.I, LEACH, D.H (1990):

Longitudinal growth analysis of horses following limited and ad libitum feeding Equine Vet. J. 22, 198-204

DALIN, G., Sandgren, B., Carlsten, J.(1993):

Plantar osteochondral fragments in the metatarsophalangeal joints in

Standardbred trotters; result of osteochondrosis or trauma?

Equine Vet J. Suppl. 16, 62-65

DÄMMRICH, K. (1985):

Wachstumsstörungen des Skeletts bei jungen Pferden

Pferdeheilkunde 1, 5-13

DER HANNOVERANER, ein Leitfaden für den Züchter, 4. Auflage, Hrsg: Verband hannoverscher Warmblutzüchter e.V.

DIAZ, C., TORO, M.A., REKAYA, R. (1999):

Comparison of restricted selection strategies: an application to selection of cashmere goats

Livest. Prod. Sci. $\underline{60}$ (1), 89-99

DIK, K.J., NEMETH, F., MERKENS, H.W. (1978):

Radiologisch-klinische Beschouwingen betreffende Podotrochlose

Tijdschr. Diergeneesk. 103, 788-797 
DIK, K.J. (1998):

Röntgenologische Befunde der Entwicklung osteochondrotischer Abweichungen im Sprung- und Kniegelenk niederländischer Warmblutfohlen im Alter von 1 bis zu 11 Monaten.

Vortrag, DVG, 15. Arbeitstagung Fachgruppe Pferdekrankheiten, Wiesbaden 52-60

DISTL, O. (2003):

Gesundheitsmerkmale in der Tierzucht

Züchtungskunde $\underline{75}$ (5), 390-400

DOHMS, T. (2002):

Einfluss von genetischen und umweltbedingten Faktoren auf die Fruchtbarkeit von Stuten und Hengsten.

Dissertation, Universität Göttingen, Fakultät für Agrarwissenschaften

EDWARDS, G.B. (1984):

Interpreting radiographs 2: The fetlock joint and pastern

Equine Vet. J. 16 (1), 4-10

ERIKSSON, J.A.(1981):

Estimating sire's breeding value for milk yield in first and second lactation by different BLUP-procedures using selected records-a simulation study In report No. 50, Dept. Anim. Breeding and Genetics, Swed. Univ. Agric. Sci., Uppsala, Sweden

EKMAN, S., CARLSON, C.S. (1998):

The pathophysiology of osteochondrosis.

Vet. Clin. North Am. Small Anim. Pract. 28, 17-32

FALCONER, D. S. (1984):

Einführung in die quantitative Genetik, 413

Verlag Eugen Ulmer GmbH, Stuttgart

FAMULA, T.R., VAN VLECK, L.D. (1982):

Monte-Carlo study of genetic groups in sire evaluation

J. Dairy Sci. $\underline{65}$, 1286-1293

FIRTH, E.C., VAN Weeren, P.R., PFEIFFER, D.U., DELAHUNT, J., BARNEVELD, A.(1999):

Effect of age, exercise and growth rate on bone mineral density (BMD) in the third carpal bone and distal radius of Dutch warmblood foals with osteochondrosis.

Equine Vet. J. Suppl. 31, 74-78

FN Jahresbericht 2004 (2005):

Deutsche Reiterliche Vereinigung und Deutsches Olympiade-Komitee

für Reiterei

FN-Verlag der Deutschen Reiterlichen Vereinigung, Warendorf 
FÖRSTER, M, BRENIG, B., BREHM, G., WITTMANN, W., LITTMANN, B., PESCHKE, W. (1992):

Der MHS-Gentest für die sichere Stressresistenzzüchtung beim Schwein.

Schweine-Zucht und Schweine-Mast, $\underline{40}$ (1), 4-6

GEBÜHRENORDNUNG FÜR TIERÄRZTE (GTO), (1999)

GELINDER, A., THOREN, E., NÄSHOLM, A., PHILIPPSON, J. (2002):

Relationships between test results at 3 and 4 years of age for Swedish riding horses. Proc. $7^{\text {th }}$ World Congress Genetics Applied to Livest. Prod., Montpellier, France, 1998

GENN, H.-J. (2005):

Persönliche Mitteilung

GERBER OLSSON, E., ARNASON, T., NÄSHOLM, A., PHILIPSSON, J. (2000):

Genetic parameters for traits at performance test of stallions and correlation with traits at progeny tests in Swedish warmblood horses

Livest. Prod. Sci. $\underline{65}, 81-89$

GLADE, M.J., GUPTA, S., REIMERS, T.J. (1984)

Hormonal responses to high and low planes of nutrition in weanling thoroughbreds

J. Animal Sci., $\underline{59}$, 658-665

GLADE, M. J., BELLING, T. H. (1986):

A dietars etiology for osteochondrotic cartilage

J. Equine Vet. Sci. $\underline{6}, 151-155$

GLAWATZ, H. (2003):

Die ökonomische Bedeutung von Osteochondrose in der Reitpferdezucht.

Master-Arbeit, Göttingen, Fakultät für Agrarwissenschaften

GLODEK, P (1979):

Merkmalsantagonismen in der Reitpferdezucht

Züchtungskunde 1ㅗ, 459-467

GLODEK und BADE (1975):

Selektionskriterien für die Pferdezucht III: Konstruktion von

Selektionsindices für eigenleistungsgeprüfte Hengste

Züchtungskunde 47, 164-171

GODDARD, M.E.,HAYES, B.J. (2002):

Optimisation of response using molecular data

$7^{\text {th }}$ World Congress on Gentics Applied to Livestock Production,

Montpellier, France 
GÖTZ, K.-U., (2001):

Logistische Vorraussetzung für die Umsetzung der markergestützten Selektion In: Genomanalyse und Markergestützte Selektion, Seminar des genetischen Ausschusses der ZAR, Geinberg, 2001

GRANEL, M. (2002):

Die Mengen- und Spurenelementversorgung von Warmblutfohlen während des ersten Lebenshalbjahres unter Berücksichtigung des Vorkommens der Osteochondrose.

Dissertation, Tierärztliche Hochschule Hannover

GRØHNDAHL, A.M, Dolvik N.I (1990):

Heritability estimations of osteochondrosis in the tibiotarsal joints and bone fragments in the metacarpo- and metatarsophalangeal joints of Norwegian Standardbred trotters.

$41^{\text {st }}$ Ann. Meeting of the EAAP, Toulouse

\section{GRØHNDAHL, A.M. (1992):}

The incidence of bony fragments and osteochondrosis in the metacarpoand metatarsophalangeal joints of Standardbred trotters.

A radiographic study.

J. Equine Vet. Sci. 12, 81-85

GUERIN, G. (2002):

Molecular genetics counselling in horse breeding

$7^{\text {th }}$ World Congress on Genetics applied to Livestock Production, Montpellier, France

GUTEKUNST, H. P. (1977):

Zur Schadensursachenstatistik von entschädigten Reitpferden in den Jahren 1971 bis 1974 innerhalb der Bundesrepublik Deutschland einschließlich Berlin-West

Dissertation, Universität Gießen, Fakultät für Agrarwissenschaften

HARING, H. J. F. (1980):

Entwicklung, Stand und Perspektiven der Pferdezucht in der Bundesrepublik Deutschland

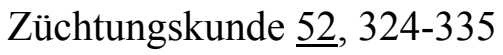

HAYES, B., GODDARD, M.E. (2003):

Evaluation of marker assisted selection in pig enterprises

Livest. Prod. Sci. $\underline{81}, 197-211$

HAZEL, L.N., LUSH, J.L. (1942):

Efficiency of three methods of selection

J. Hered. 33 
HEINZ, T.C. (1993):

Röntgenologische Reihenuntersuchung an den Zehen und Sprunggelenken

Holsteiner Fohlen

Dissertation, Tierärztliche Hochschule Hannover

HENDERSON, C.R. (1973):

Sire evaluation and genetic trends

Proc. Anim. Breeding and Genetics

Symp. in Honor of Dr. J. L. Lush.

ASAS and ADSA, Champaign, Illionois, 10-41

HERTSCH, B., KROLL, A. (1999):

Skelettreife beim Fohlen

Göttinger Pferdetage 1999

HERTSCH, B., HÖPPNER, S. (1999)

Gelenkchirurgie beim Pferd-Steine werden aus dem Weg geräumt

Pferdeheilkunde 15, 159-166

HERTSCH, B. (2000):

Die Gelenkerkrankung des Fohlens

Die moderne Sportpferdezucht, Verden, 39-42

HERTSCH, B. (2002):

Die Osteochondrosis dissecans

Pferdeworkshop Uelzen, 2002

HOEKSTRA, K.E., NIELSEN, B.D., ORTH, M.W., ROSENSTEIN, D.S., SCHOTT, H.C., SHELLE, J.E. (1999):

Comparison of bone mineral content and biochemical markers of bone metabolism in stall vs. pasture-reared horses

Equine Vet. J. Suppl. 30, 601-604

HOFMANN, R., SCHÖNBAUER, M. (1981):

Zur Diagnose der Osteochondrosis dissecans des Pferdes

Berl. Münch. Tierärztl. Wschr. 95, 26-30

HOMMERICH, G. (1995):

Ausfallursachen und -frequenzen

In: Göttinger Pferdetage 1995 - Zucht und Haltung von Sportpferden.

HOPPE, F. (1984):

Radiological investigations of osteochondrosis dissecans in

Standardbred Trotters and Swedish warmblood horses.

Equine Vet. J. $\underline{5}$, 425-429

HUIZINGA, H.A. (1990):

Genetic studies on performance of the Dutch warmblood riding horse

Dissertation Wageningen-Utrecht 
HULTGREN, B.D., APPELL, L.H., WAGNER, P.C., BLYTHE, L.L., WATROUS, B.J., SLIZESKI, M.L., DUFFIELD, D.A., GOLDIE, P, CLARKSON, D, SHELL, D. (1988):

Current research topics in equine genetics, part 2

Equine practise 10, 19-22

HURTIG, M.B., GREEN, S.L., DOBSON, H., MIKUNI-TAKAGAKI, Y., CHOI, J. (1993):

Correlative study of defective cartilage and bone growth in foals fed a low-copper diet

Equine Vet. J., 16, 66-73

JEFFCOTT, L.B. (1991):

Osteochondrosis in the horse - searching for the key to pathogenesis.

Equine Vet. J. 23, $331-338$

JEFFCOTT, L.B. (1993):

Problems and pointers in Osteochondrosis

Equine Vet. J. Suppl. 16, 1-3

JEFFCOTT, L.B. (1996):

Osteochondrosis: an international problem for the industry

J. Equine Vet. Sci., 16, 32-37

JEFFCOTT, L.B., SAVAGE, C.J. (1996):

Nutrition and the development of Osteochondrosis (Dyschondroplasia)

Pferdeheilkunde $\underline{12}$, 338-342

JEFFCOTT, L.B.(1997):

Osteochondrosis in horses

In practise, 64-71

JEFFCOTT, L.B., BUCKINGHAM, S.H., MCCARTHY R.N., CLEELAND J.C., SCOTTI E., MCCARTNEY R.N. (1988):

Non-invasive measurement of bone: a review of clinical and research applications in the horse.

Equine Vet. J. Suppl. ㅁ: 71-79

JEFFCOTT, L.B., HENSON, F.M.D. (1998):

Studies on growth cartilage in the horse and their application to aetiopathogenesis of dyschondroplasia (Osteochondrosis)

Vet. J. $156,177-192$

JEFFCOTT, L.B. (2004):

Developmental diseases affecting growing horses In: EAAP Publication No. 114, The growing horse: nutrition and prevention of growth disorders, Wageningen Academic Publishers, 2005 
JELAN, Z.A., JEFFCOTT, L.B., LUNDEHEIM, N., OSBORNE, M. (1996):

Growth rates in thoroughbred foals

Pferdeheilkunde 12, 291-295

KALM, E. (2002):

Beispiel zur Gesundheitsdatenerfassung

Tagungsband Uelzener Pferdeworkshop, 2002

KARSTEN, S., KRIETER, J. (2002):

Simulationsstudie zur Verbreitung der Schweinepest

Vortragstagung der DGfZ/GfT, Halle

KATONA, Ö., DISTL, O., KRÄUSSLICH, H. (1983):

Zuchtwertschätzung mittels der BLUP-Methode in der Pferdezucht

IV. Int. Wiss. Symposium, Leipzig, 223-235

KELM, S.C., FREEMAN, A.E. (2000):

Direct and correlated responses to selection for milk yield:results and conclusion of regional project NC-2, Improvement of dairy cattle trough breeding, with emphasis on selection

J. Dairy Sci. $\underline{83,2721-2732}$

KOENEN, E.P.C., DIK, K.J., KNAAP, J.H., VAN DER KUIL, R.J.G., VAN WEEREN, P.R.(2000):

Evaluation of selection strategies against osteochondrosis for the Dutch warmblood riding horse population

$51^{\text {st }}$ Annual Meeting of EAAP, The Hague

KÖNING, B. (1983):

Vergleichende Untersuchungen über das Alter, die Versicherungsdauer und die Abgangsursachen versicherter Pferde bei Eintritt des Schadensfalls auf der Grundlage von Schadensstatistik verschiedener Versicherungen und Jahre Dissertation, Universität Gießen, Fakultät für Agrarwissenschaften

KRÄUSSLICH, H. (1994):

Tierzüchtungslehre

4. Auflage, Ulmer Verlag, Stuttgart

KROLL, A. (2000):

Zur Osteochondrosis dissecans beim Saugfohlen

Dissertation, freie Universität Berlin, Fakultät für Veterinärmedizin

KWPN (1994):

The frequency and heredity of navicular disease, sesamoidosis, fetlock joint arthrosis, bone spavin, osteochondrosis of the hock. A radiographic progeny study

KWPN, Zeist 
KÜNZI, N., STRANZIGER, G. (1992):

Allgemeine Tierzucht, 72

1. Aufl., Verlag Ulmer, Stuttgart

LAWS, E.G., RICHARSON, D.W., ROSS, M.W., MOYER, W. (1993):

Racing performance of standardbreds after conservative and surgical treatment for tarsocrural ostechondrosis

Equine Vet. J. 25 (3), 199-202

LEE, G.J., ARCHIBALD, A.L., GARTH, G.B., SAW, A.S., NICHOLSON, D., BARR, A., HALEY, C.S. (2003):

Detection of quantitative trait loci for locomotion and osteochondrosis- related traits in Large White x Meishan pigs

Animal Science, 76, 155-165

LENSING, M. (1998):

Eine Pilotstudie zum Einfluss der Fütterung auf Knochenmarker beim Pferd

Dissertation, Universität München; Fakultät für Veterinärmedizin

LEPAGE, O.M., MARCOUX, M., TREMBLAY, A. (1990):

Serum osteocalcins, or bone Gla-protein, a biochemical marker for bone metabolism in horses: differences in serum level with age

CAN. J. VET. Res., 54, 223-226

LEPAGE, O.M., CARSTANJEN, B., UEBELHART, D. (2001):

Non-Invasive Assessment of Equine Bone: An Update

The Vet. Journal 2001, 161, 10-23

LEWIS, L.D. (1989):

Einfluss der Ernährung auf die Entwicklung des Bewegungsapparates und seine Erkrankungen In: Adam's Lahmheiten bei Pferden, 4. Auflage

Verlag M.\& H. Schaper, Hannover, 271-286

LILLICH, J.D., BERTONE, A.L., MALEMUD, C.J., WEISBRODE, S.E., RUGGLES, A.J., STEVENSON, S. (1997):

Biochemical, histochemical and immunohistochemical charecterization of distal tibial osteochondrosis in horses Am. J. Vet. Res., $\underline{58}$, 89-98

LIESEGANG, A. (2000):

Anwendung von Knochenmarkern in der Veterinärmedizin

Schweiz, Arch. Tierheilkd., Band 142, Heft 11, 613-623

LINDNER; A, OFFENEY, F. (1992):

Einsatzdauer, Abgangsraten und Ursachen bei Sportpferden

Dtsch. Tierärztl. Wochenschr., 99, 39-42 
LÖHRING, K. (2003):

Genome scan for Quantitative Trait Loci (QTL) for osteochondrosis

in Hanoverian warmblood horses using an optimised microsatellite marker set.

Dissertation, Tierärztlich Hochschule Hannover

LOOFT, C. (2002):

Stand der Diagnostik beim Pferd

Pferdeworkshop Uelzen, 143-149

LUSH, J. (1945):

Animal Breeding Plans

Aimes, Iowa

LÜHRS-BEHNKE, H. (2002):

Aktuelle Fragen der Pferdezucht von der Zuchtwertschätzung bis zur

Tiergesundheit, Tagungsband Uelzener Pferdeworkshop 2002

MICHEL,G. (1983):

Kompendium der Embryologie der Haustiere

Vierte, überarbeitete Auflage, Gustav Fischer Verlag, Stuttgart, New York

MAHRAHRENS, F. (1993):

Definition des Zuchtziels

Pferdeworkshop Uelzen, 111-113

MC ILWRAITH, (1986):

Incidence of developmental joint problems.

In: AQHA Developmental Orthopaedic Disease

Symposium, Amarillo, Texas, 15-20

MEINARDUS, H. (1988):

Züchterische Nutzung der Turniersportprüfung für Reitpferde

Dissertation, Universität Göttingen, Fakultät für Agrarwissenschaften

MEUWISSEN, T.H.E., VAN DER WERF, J.H.J. (1993):

Impact of heterogeneous within herd variances on dairy cattle breeding schemes : a simulation study

Livest. Prod. Sc. $\underline{33}$ (1-2), 31-41

MEYER, H. (1996):

Das neugeborene Fohlen- alles startklar?

Pferdeheilkunde 12: 171-178

MIESNER, K. (1996):

Untersuchungen über die abduktorische Funktionsstörung des Kehlkopfs beim Pferd-Genetische und umweltbedingte Einflussfaktoren sowie mögliche züchterische Maßnahmen

Dissertation, Universität Bonn, Fakultät für Agrarwissenschaften 
NICHOLAS, F.W. (2000):

Genetics of morpological traits and inherited disorders.

The Genetics of the horse

CAB Int'1, 71-84

NILSON (1947):

Gonitis in the horse

Svensk Veterinätid Skrift $\underline{52}, 1-14$

NISSEN, T. (1986):

Stutenprüfung beim Holsteiner Warmblutpferd-Leistungsveranlagung und Fruchtbarkeit

Dissertation, Universität Kiel, Fakultät für Agrarwissenschaften

NISSEN, T. (2002):

Erfahrungen mit der Stutenprüfung und zukünftige Ausrichtung.

Tagungsband 3. Pferdeworkshop, Uelzen

NIXON, A.J., (1993):

Die Oberfläche des Gelenkknorpels: Struktur und Funktion

Pferdeheilkunde $\underline{9}, 95-100$

NRC (National Research Council) (1989):

Nutrient requirement of horses

$5^{\text {th }}$ edition, National Academy Press, Washington, DC

OEXMANN, B. (2004):

Pflichtverletzung durch Hengsthalter und Zuchtverbände bei OCD

In: Z-Magazin BV, Postbus 2066, NL-6201 cd, Maastricht

PAGAN, J.D., Jackson, S.G., (1996):

The incidence of developmental orthopaedic disease on a Kentucky thoroughbred Farm

Pferdeheilkunde $\underline{12}$, 351-354

PETZOLD, P, KÖNIG, S., SCHWARK, H.J. (1988):

Abgangsursachen von englischen Vollblütern aus dem Renngeschehen

Leipziger Symposium, 114-126

PHILIPSSON, J., ANDREASSEN, E., SANDGREN, B., DALIN, G., CARLSTEN, J. (1993):

Ostechondrosis in the tarsocrural joint and osteochondral fragments in the fetlock joint an standardbred trotters. II. Heritability

Equine Vet. J., Suppl. 16, 38-41

PHILIPSSON, J., BRENDOV, E., DALIN, G., WALLIN, L. (1998):

Genetic aspects of diseases and lesions in horses

World Conference on Genetics applied to Livestock Production,

Armidale, Australia 24, 408-415 
PHILIPSSON, J. (1999):

Genetische Aspekte von Erkrankungen beim Pferd

Göttinger Pferdetage 1999

PIERAMATI, C., PEPE, M., SILVESTRELLI, M., BOLLA, A. (2003):

Heritability estimation of osteochondrosis dissecans in

Maremanno

Livestock Prod. Sci. $\underline{79}$, 249-255

PIRCHNER, F. (1979):

Populationsgenetik in der Tierzucht

Verlag Paul Parey

POOL, R. (1993):

Difficulties in definition of equine osteochondrosis; differentation of developmental and aquired lesions

Equine Vet. J., Suppl. 16, 5-12

PRICE, J.S., JACKSON, B.F., GRAY, J.A., HARRIS, P.A., WRIGHT, I.M., PFEIFFER, D.U., ROBINS, S.P., EASTELL; R., RICKETTS, S.W. (2001):

The response of skeleton to physical training: a biochemical study in horses Bone 1995, 17, 221-227

PRICE, J.S., JACKSON, B.F.,GRAY, J.A., HARRIS, P.A.,WRIGHT, I.M., PFEIFFER, D.U., ROBINS, S.P., EASTELL, R., RICKETTS, S.W. (2001):

Biochemical markers of bone metabolism in growing thoroughbreds: a longitudinal study

Res. Vet. Sc., $\underline{71}, 37-44$

RALSTON, S.L. (1997):

Feeding the rapidly growing foal

J. Equine Vet. Sci. 17, 634-636

RAUF, G. (1964):

Literaturstudie über Ursache, Diagnostik und Behandlung des

Kehlkopfpfeifens der Pferde

Dissertation, Tierärztliche Hochschule Hannover

REINSCH, N. (2002):

Einbeziehung von Markern in die Zucht

Pferdeworkshop Uelzen, 150-157

RICARD, A, VALETTE, J.P., DENOIX, J.M. (2002):

Heritability of juvenile osteo-articular lesions of sport horses in France $7^{\text {th }}$ World Congress on Genetics Applied to Livestock

Production,Montpellier 
RICARD, A. (2004):

Genetic backgrounds of osteochondrosis

$55^{\text {th }}$ EAAP Meeting, Bled, Slovenia

ROBERT, C., VALETTE, J.-P., JAQUET, S., DENOIX, J.-M. (2005):

Breed variations in the distribution of osteo-articular lesions in horses at weaning, $56^{\text {th }}$ Annual Meeting of EAAP, Uppsala, Sweden

ROBINSON, R. (1989):

Genetic defects in the horse

J. Anim. Breed. Genetic. 106, 475-478

RÖNNINGEN, K. (1970):

Studies on selection in animal breeding V. Bias in the estimates of the genetic correlation due to selection according to an unrestricted selection index

Acta Agric. Scand. 20, 143-161

SALDERN, F. CH, von (2002):

Die röntgenologische Bewertung bei Ankaufsuntersuchungen

Tagungsband, Uelzener Pferdeworkshop, 2002

SAMY, M.T. (1977):

Osteochondrosis dissecans bei Mensch Hund und Pferd.

Dissertation Tierärztliche Hochschule Hannover

SANDGREN, B., DALIN, G., CARLSTEN, J. (1993):

Osteochondrosis in the tarsocrural joint and osteochondral

fragments in the fetlock joint of standardbred trotters

I. Epidemiology

Equine Vet. J. Suppl. 16, 31-37

SAVAGE, C.J., MCCARTHY, R.N., JEFFCOTT, L.B. (1993):

Effects of dietary energy and protein on induction of dyschondroplasia in foals

Equine Vet. J. Suppl. 16, 89-93

SCHADE, W. (1996):

Entwicklung eines Besamungszuchtprogramms für die

Hannoversche Warmblutzucht

Dissertation, Universität Göttingen, Fakultät für Agrarwissenschaften

SCHOBER, M. (2003):

Schätzung von genetischen Effekten beim Auftreten von

Osteochondrosis dissecans beim Warmblutpferd.

Dissertation, Universität Göttingen

SCHOUGAARD, H., FALK-RONNE, J. PHILIPSSON, J. (1987):

Osteochondrosis in the tibio-tarsal articulation of trotters and its disposition $38^{\text {th }}$ Annual Meeting of EAAP, Lissabon 
SCHRYVER, H.F., MEAKIM, D.W., LOWE, J.E., WILLIAMS, J., SODERHOLM, L.V., HINTZ, H.F., (1987):

Growth and calcium metabolism in horses fed varying levels of protein

Equine Vet. J.,19, 280-287

SCHÜLER, L., SWALVE, H., GÖTZ, K.-U. (2001):

Grundlagen der quantitativen Genetik,173, 232, 234,

Verlag Eugen Ulmer

SWALVE, H. (1984):

Untersuchungen zur Anwendung der Verwandtschaftsmatrix bei der

Zuchtwertschätzung nach der BLUP-Methode

Dissertation, Göttingen, Fakultät für Agrarwissenschaften

SWALVE, H., BRUNS, E. (1986):

Considering relationships and genetic grouping in mixed model sire

evaluation: A simulation study

Livest. Prod. Sci. 14 (2), 123-138

SCHWARK, H.J.(1988):

Pferdezucht, 3. Aufl.

BLV Verlagsgesellschaft mbH, München

SCHWARZENBACH, H., VISSCHER, DOLEZAL, M., SOLLER, M., SÖLKNER, J. (2004).

Simulationsstudie zur Marker gestützten Selektion mit komplexem Zuchtziel beim Rind

Vortragstagung DGfZ/GfT, Rostock

SCHWEDE, H. (1987):

Knochenreifungsstörungen als möglicher leistungsmindernder

Faktor bei jungen Pferden

Monatsh. Veterinärmedizin, $\underline{42}$, 263-266

SEIBEL, M.J., WOITGE, H.W., ZIEGLER, R. (1993):

Biochemische Marker des Knochenstoffwechsels I: Grundlagen

Klein. Lab., 39, 717-727

SHINGLETON, W.D., MACKIE, E.W., CAWSTON, T.E., JEFFCOTT, L.B. (1997):

Cartilage canals in equine articular/ epiphyseal growth cartilage and a possible association with dyschondroplasia

Equine Vet. J., 29, 360-364

SLOET VAN OLDRUITENBORGH-OOSTERBAAN, M.M,

BARNEVELD, A (1999):

Hormones, growth factors and other plasma variables in relation to

osteochondrosis.

Equine Vet. J. Suppl. $\underline{31}$, 45-54 
STÄCKER, W., BREHM, W. (1994):

OCD im Tarsokruralgelenk beim Trabrennpferd- Heritablititätsschätzung anhand röntgenologischer Befunde

Proc. Int. Symposium joint diseases in the horse,

Dortmund, 99-105

STASHAK, T.S. (1989):

Lahmheit

In: Adam's Lahmheit bei Pferden, 4. Auflage

Verlag M. \& H. Schaper

STOCK, K.F. (2004):

Radiographic findings in the limbs of Hanoverian warmblood horses:

Genetic analyses and relationship with performance in sports

Dissertation, Tierärztliche Hochschule Hannover

STOCK, K.F., HAMANN, H., DISTL. O. (2004):

Genetische Beziehungen zwischen röntgenologischen Gliedmaßenbefunden und dem Einsatz der Pferde im Reitsport

Vortragstagung der DGfZ und GfT, Rostock

STÖCKLI, M, UELTSCHI, G. (1992):

Radiologische Untersuchung am Fesselgelenk klinisch gesunder und lahmer Pferde

Pferdeheilkunde $\underline{8}$ (4), 209-214

STORGAARD JØRGENSEN, PROSCHOWSKY, H, FALK -RØNNE, J (1997):

The significance of routine radiographic findings with respect

to subsequent racing performance and longlivety in

standardbred trotters.

Equine Vet. J. 29, 55-59

STROMBERG; B., REJNO, S. (1978):

Osteochondrosis in the horse. I: A clinical and radiological investigation of osteochondrosis of the knee and hock joint

Acta. Radiolo. Suppl., 358, 139-152

STROMBERG; B. (1979):

A review of salient features of osteochodrosis in the horse

Equine Vet. J., 11, 211-214

SULLIVAN, P.G., WILTON, J.W., SCHAEFFER, L.R., JANSEN, G.J., ROBNISON, J.A.B., ALLEN, O.B. (2005):

Genetic evaluation strategies for multiple traits and countries

Livest. Prod. Sci. 92, 195-205 
SWINBURNE, J., GERSTENBERG, C., BREEN, M., ALDRIDGE, V., LOCKHARDT, L., MARTI, E., ANTZACK, D., EGGESTON-STOTT, M., BAILEY, E., MICKELSON, J., ROED, K., LINDGREN, G., VON HAERINGEN, W., GUERIN, G., BJARNASON, J., ALLEN, T., BINNS, M. (2000): First comprehensive low-density horse linkage map based on two 3-generation, full sibling, cross-bred, horse reference families Genomics, $\underline{66}, 123-134$

TAVERNIER, A. (1990):

Estimation of breeding value of jumping horses from their ranks Livest. Prod. Science 26, 277-290

THOMPSON, K.N. (1995):

Skeletal growth rates of weanling and yearling thoroughbred horses.

J. Anim. Sci. 73, 2513-2517

THOMPSON, K.N., BAKER, J.P, Jackson, S.G (1988):

The influence of supplemental feed on growth and bone development of nursing foals.

J. Anim. Sci. $\underline{66}, 1692-1696$.

THOREN, E., GELINDER, A., KOENEN, E., RICARD, A., BRUNS, E., PHILIPSSON, J. (2005):

Review of genetic parameters estimated at stallion and young horse performance tests and their correlations with later results in dressage and show jumping competition

Livestock Production Science, in Vorbereitung

UPHAUS, H. (1993):

Feld- und Stationsprüfung für Stuten und deren Nutzung im Rahmen eines Zuchtprogramms

Dissertation, Universität Kiel, Fakultät für Agrarwissenschaften

VAN HOOGMOED, L.M., SNYDER, J.R., THOMAS, H.L., HARMON, F.A. (2003):

Retrospective evaluation of equine prepurchase examinations performed 1991-2000

Equine Vet J. $\underline{35}$ (4), 375-381

VAN VELDHUIZEN, A.E., (1995):

Reitpferdezucht in Holland.

In: Tagungsband Göttinger Pferdetage , 1995

VAN WEEREN, P.R., SLOET VAN OLDRUITENBORGH-OOSTERBAAN, M.M, BARNEVELD, A (1999):

The influence of birth weight, rate of weight gain and final achieved height and sex on the development of osteochondrotic lesions in a population of genetically predisposed warmblood foals.

Equine Vet. J. Suppl. $\underline{31}$, 26-30 
VAN WEEREN; P.R. (2002):

New thoughts on the background of OCD

Seminar: Managing growth rates to reduce skeletal disease. Newmarket

VAN WEEREN, P.R. (2004):

Grundlagen der Gelenkentwicklung und- erkrankung wachsender Pferde

Tagungsband Göttinger Pferdetage, 2004

VELSEN-ZERWECK, A. von (1998):

Integrierte Zuchtwertschätzung für Zuchtpferde

Dissertation, Universität Göttingen, Fakultät für Agrarwissenschaften

VELTJENS, C. (1987):

Der Einfluss endogener und exogener Faktoren des Bewegungs-und Atmungsapparates bei Pferden verschiedener deutscher Zuchtgebiete Dissertation Universität Bonn, Fakultät für Agrarwissenschaften

WAGNER von MATTHIESEN (1994):

Equine osteochondrosis review and update

J. Equine Vet. Sci. 14, 72-74

WALLIN, L., STRANDBERG, E., PHILIPSSON, J., DALIN, G. (2000):

Estimates of longevity and causes of culling and death in Swedish warmbloods and coldblood horses

Livest. Prod. Sci. $\underline{63}$, 275-289

WILLIAM, A. (2001):

Markergestützte Selektion in Rinderzuchtprogrammen

In: Genomanalyse und Markergestützte Selektion, Seminar des genetischen

Ausschusses der ZAR, Geinberg, 2001

WILLARD, J:G., WILLIAMS, J.L., WILLARD, J.C., KNAPP, J.R., GREENE, E.A. (2004):

The effect of dam on birth weigt and growth rate of thoroughbred foals In: EAAP Publication No. 114, The growing horse: nutrition and prevention of growth disorders, Wageningen Academic Publishers, 2004

WILKE, A. (2003):

Der Einfluss von Haltung und Aufzucht auf die Häufigkeit von Osteochondrose

Dissertation, Tierärztliche Hochschule Hannover

WILLMS, F. (1998):

Genetische Analyse von Merkmalskomplexen der Reitpferdezucht unter Berücksichtigung von Gliedmaßenveränderungen.

Dissertation Universität Kiel, Fakultät für Agrarwissenschaften

WINKELSETT, S. (2003):

Biochemische Knochenmarker und Parathormon bei Warmblutfohlen unter

Berücksichtigung des Vorkommens der Osteochondrose

Dissertation, Tierärztliche Hochschule Hannover 
WINTER, D. (1995):

Genetische Disposition von Gliedmaßenerkrankungen bei Reitpferden.

Dissertation, Universität Göttingen, Fakultät für Agrarwissenschaften

WITTWER, C.,BÖNEKER, C., HAMANN, H., DISTL, H. (2004):

Genetische Analyse der Osteochondrose beim Süddeutschen Kaltblut Vortragstagung der DGfZ und GfT, Rostock

WEYMANN, W. (1992):

Zuchtwertschätzung für Exterieurmerkmale am Beispiel der Hannoverschen Warmblutzucht

Dissertation, Universität Göttingen, Fakultät für Agrarwissenschaften

ZELLER, R., HERTSCH, B., SAMY, M.T. (1978):

Vorkommen und klinische Beurteilung der Osteochondrosis dissecans (O.d.) im Talokruralgelenk beim Pferd

Dt. tierärztl. Wschr. $\underline{85}, 189-272$

ZUCHTVERBANDSORDNUNG (ZVO), (2005).

Besondere Bestimmungen- Rahmenbestimmungen für die Population in der deutschen Reitpferdezucht, $\S 200$ 


\section{Danksagung}

Mein besonderer Dank richtet sich an Herrn Prof. Dr. E. Bruns für die Überlassung des Dissertationsthemas und für die jederzeit gewährleistete Unterstützung, Betreuung und Diskussion. Für die Übernahme des Korreferats richtet sich mein Dank an Herrn Prof. Dr. Dr. M. Gauly.

Herrn Dr. L. Christmann, Herrn Dr. J. Wilkens und Herrn U. Hahne danke ich für wertvolle, wegweisende Hinweise zu Beginn der Arbeit.

Ein großer Dank gilt auch den Mitarbeitern und Mitdoktoranden des Instituts für Tierzucht und Haustiergenetik. Für die jederzeitige Unterstützung danke ich Burchhard Möllers sowie Sven König und Manfred Tietze für die Hilfe bei Programmierproblemen. Meinem Büronachbarn Yagmur Akgün danke ich für ein humorvolles und schönes Miteinander. Bianca Lind sei gedankt für viele lustige gemeinsame Mittagspausen.

Ebenso herzlich danken möchte ich meinen Eltern und Christian Reyher für die Unterstützung in jeglicher Hinsicht und aufbauenden Worte in dieser Zeit. Auch meinen Freunden gebührt großer Dank für die Unterstützung und Bestärkung. 


\section{Lebenslauf}

Name

Geburtstag und-ort

Staatsangehörigkeit

Familienstand

Eltern

Schule

1981-1983

1983-1985

1985-1991

1991-1995
Stephanie-Mabinti Busche

13.03.1975 in Freetown/Sierra Leone

deutsch, belgisch

ledig

Hans-Jürgen Busche, Bauingenieur

Lena-Maria Busche, geb. Beyne

\section{Landwirtschaftliches Praktikum}

04/2000-06/2000

07/2000-09/2000

\section{Studium}

1997-2002
Grundschule Laudenbach

Grundschule Xanten

Mädchenrealschule Xanten

Städtisches Stiftsgymnasium Xanten

Abschluss: Abitur

Betrieb F. Aichholzer, St. Egyden, Kärnten

Betrieb T. Hinckers, Uedem

Studium der Agrarwissenschaften an der Justus - LiebigUniversität, Gießen, Fachrichtung: Tierproduktion Abschluss: Dipl.-Ing. agr.

\section{Berufstätigkeit}

1995-1997

Ausbildung zur Tierarzthelferin in der Pferdeklinik Dres. Genn und Steinmann

2003-2005 Anfertigung der vorliegenden Dissertation am Institut für Tierzucht und Haustiergenetik der Universität Göttingen 